\title{
LIGHT-SCATTERING CHARACTERISTICS \\ OF OPTICAL SURFACES
}

\author{
by
}

James Elmer Harvey

\begin{abstract}
A Dissertation Submitted to the Faculty of the COMMITTEE ON OPTICAL SCIENCES (GRADUATE)

In Partial Fulfillment of the Requirements

For the Degree of

DOCTOR OF PHILOSOPHY

In the Graduate College

THE UNIVERSITY OF ARIZONA
\end{abstract}

197.6 
(c) $\quad 1976$

JAMES ELMER HARVEY

\section{ALL RIGHTS RESERVED}




\section{THE UNI VERSITY OF ARIZONA}

GRADUATE COLTEGE

I hereby recommend that this dissertation prepared under my direction by James Elmer Harvey

entitled Light-Scattering Characteristics

of Optical Surfaces

be accepted as fulfilling the dissertation requirement for the degree of Doctor of Philosophy

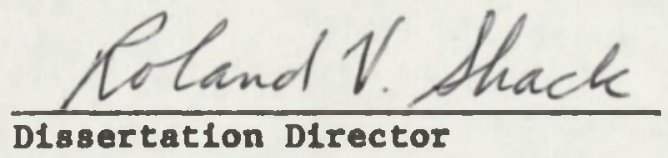

$\frac{1 \text { OCT } 76}{\text { Date }}$

As members of the Final Examination Comittee, we certify that we have read this dissertation and agree that it may be presented for final defense.
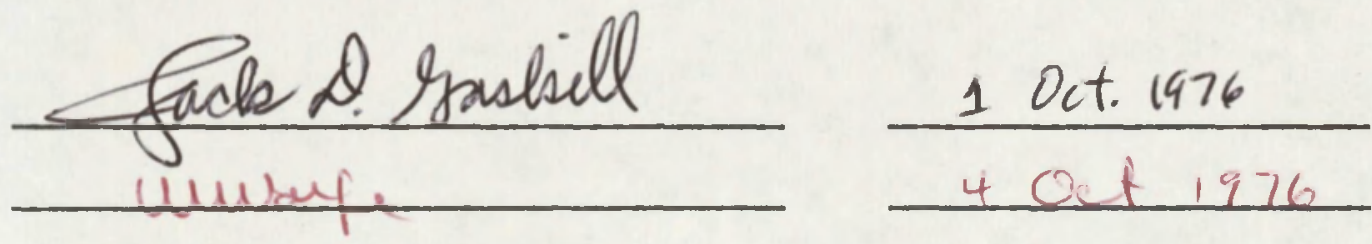

Final approval and acceptance of this dissertation 18 contingent on the candidate's adequate performance and defense thereof at the final oral examination. 


\section{STATEMENT BY AUTHOR}

This dissertation has been submitted in partial fulfillment of requirements for an advanced degree at The University of Arizona and is deposited in the University Library to be made available to borrowers under rules of the Library.

Brief quotations from this dissertation are allowable without special permission, provided that accurate acknowledgment of source is made. Requests for permission for extended quotation from or reproduction of this manuscript in whole or in part may be granted by the copyright holder.

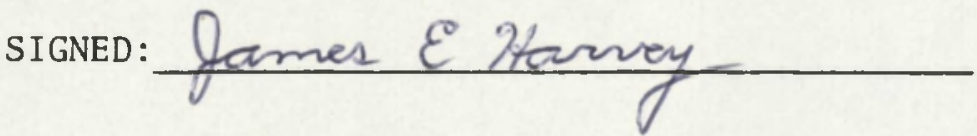




\section{ACKNOWLEDGMENTS}

The author wishes to extend his appreciation to the entire faculty and staff of the Optical Sciences Center for their assistance and support over the past few years of study and research. Special thanks are due to Dr. Roland V. Shack for the helpful suggestions, technical insight, and constructive criticism he contributed during the course of this program.

The stimulating discussions and encouragement provided by many friends and fellow students played an important role in the successful completion of this project.

This research was supported by the U. S. Air Force Space and Missile Systems Organization under Contract Nos. F04701-74-C-0083 and F04701-75-C-0106.

The continued encouragement and moral support offered by my wife, Marva, throughout the entire course of my graduate program is also gratefully acknowledged. 
TABLE OF CONTENTS

Page

LIST OF ILLUSTRATIONS . . . . . . . . . ...... . vii

LIST OF TABLES . . . . . . . . . . . . . . . . xi

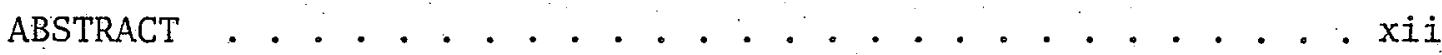

1. INTRODUCTION ................................. 1

Background .. . . . . . . . . . . . . . . . . . . 1

Dissertation Content .............. . . . 4

2. A FOURIER TREATMENT OF NEAR-FIELD SCALAR DIFFRACTION THEORY . • 6

The Diffracted Wave Field as a Superposition of Plane

Waves . . . . . . . . . . . . . . 7

Initial Conditions . . . . . . . . . . . . 7

The Direct Application of Fourier Transform Theory . . 9

The Transfer Function of Free Space . . . . . . . . . 10

The Diffracted Wave Field as a Superposition of Spherical

Waves ................... 14

The Point Spread Function .. . . . . . . . . . 14

Huygens' Principle . . . . . . . . . . . . . . 15

General Rayleigh-Sommerfeld Diffraction Formula . . . 16

Geometrical Configurations of the Observation Space . . . . 17

The Diffracted Wave Field on a Plane . . . . . . . 17

The Diffracted Wave Field on a Hemisphere . . . . . . 19

Aberrations of Diffracting Systems . . . . . . . . . . 22

Shift Invariance of the Diffracted Wave Field. . . . . . 28

Summary ................. . . 32

3. SURFACE SCATTER THEORY . . . . . . . . . . . . . 35

Surface Scatter Phenomena as a Diffraction Process . . . . 35

The System Pupil Function ........... . . 36

Intensity Distribution of a Scattering System . . . . 38

The System Spread Function . . . . . . . . . . . 39

Scattering from Optical Surfaces ............. . 39

The Statistical Properties of an Optical Surface . . . 41

The Transfer Function of a Scattering Surface . . . . 42

The Spread Function of a Scattering Surface . . . . . 45 
TABLE OF CONTENTS--Continued

Page

A Shift-invariant Scattering Function....... 47

The Inverse Scattering Problem . . . . . . . . . . 50

Wavelength Dependence of the Scattering Function . . 52

4. SURFACE SCATTER MEASUREMENTS . . . . . . . . . . . 55

Apparatus . . . . . . . . . . . . . . . . 55

The Light Source . . . . . . . . . . . . 55

The Detector Unit.... . . . . . . . . . 58

The Scanning Mechanism ............ 64

The Incident Beam . . . . . . . . . . . . 66

Experimenta1 Procedures . . . . . . . . . . . 70

Sample Preparation ............. . . 71

System Alignment . . . . . . . . . . . . 73

Measurement Technique . . . . . . . . . . 75

Presentation of the Data ............ 76

5. DATA AND RESULTS ................... 85

Surface Roughness Effects... . . . . . . . . 85

Incident Angle Effects . . . . . . . . . . . . 88

The Inverse Scattering Problem. . . . . . . . . . . . 94

Predictions of Surface Characteristics . . . . . . . 94

Surface Structure Measurements . . . . . . . . . . 102

Comparison of Predictions with Measurements . . . . 106

Indirect Verification of Inverse Scattering

Solution ................ . 109

Wavelength Effects .............. 111

6. CONCLUSION . . . . . . . . . . . . . 116

Summary of Results . . . . . . . . . . . . . . 116

Further Theoretical Considerations . . . . . . . . . 118

Suggestions for Further Research . . . . . . . . . 121

APPENDIX A: CALCULATION OF ABERRATION COEFFICIENTS FOR

DIFFRACTED WAVE FIELDS ............ 122

APPENDIX B: BIDIRECTIONAL REFLECTANCE DISTRIBUTION

FUNCTION . . . . . . . . . . . . . 127

APPENDIX C: COMPUTER PROGRAM FOR INVERSE SCATTERING

PROBLEM . . . . . . . . . . . . . 130 
TABLE OF CONTENTS--Continued

Page

APPENDIX D: COMPUTER PROGRAM FOR PROCESSING SURFACE

PROFILE DATA FROM ELECTRON-MICROGRAPH

STEREOCOMPARATOR . . . . . . . . . 137

APPENDIX E: DESCRIPTION OF SCANNING FECO INTERFEROMETER

USED FOR DETERMINING STATISTICAL PROPERTIES

OF OPTICAL SURFACES . . . . . . . . . 140

LIST OF REFERENCES .................. . . 155 


\section{LIST OF ILLUSTRATIONS}

Figure

1.1. Schematic Representation of Reflectance from a Rough Surface . . . . . . . . . . . . . . . . . . 2

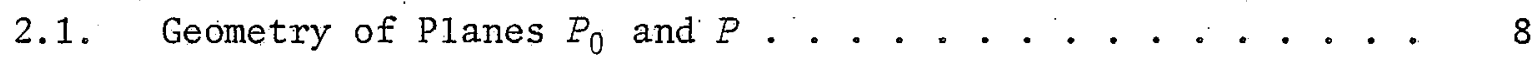

2.2. Unit Circle in Direction Cosine Space . . . . . . . . . 12

2.3. Illustration of the Propagating and Evanescent Parts of the Diffracted Wave Field . . . . . . . . . . 13

2.4. Geometrical Relationship between Incident Beam, Diffracting Aperture, and Observation Hemisphere ... . 20

2.5. Diffraction Pattern of a 10-Line per mm Ronchi Ruling

Placed in an $\mathrm{f} / 6$ Cone of Light with a $40-\mathrm{mm}$

Diameter . . . . . . . . . . . . . . . . . . .

2.6. Diffraction Pattern of a 10-Line per mm Ronchi Ruling

Placed in an $f / 20$ Cone of Light with a $12-\mathrm{mm}$

Diameter . . . . . . . . . . . . . . . .

2.7. Geometrical Configuration when the Incident Beam

Strikes the Diffraction Aperture at an

Arbitrary Angle . . . . . . . . . . . . . . . .

2.8. Illustration of the Position of the Diffracted Orders in

Real Space and Direction Cosine Space . . . . . . . . .

3.1. Illustration of Surface Height Variations and

Associated Statistical Parameters . . . . . . . . .

3.2. An Ensemble of Two-Dimensional Sample Functions

$\hat{W}_{i}(\hat{x}, \hat{y})$ Representing Surface Height Variations

Constitutes a Random Process $\hat{W}(\hat{x}, \hat{y})$. . . . . . . . .

3.3. Illustration of the Effective Transfer Function and Spread Function of a Scattering Surface . . . . . . . 
viii

LIST OF ILLUSTRATIONS--Continued

Figure

Page

3.4. Geometrical Relationship between Incident Beam,

Scattering Surface, the Measured Scattered Light

Distribution, and the Resulting Spread Function . . . .

4.1. Schematic Diagram of Scatter Measurement Apparatus . . . 56

4.2. Apparatus for Measuring Scattered Light Distribution

from Optical Surfaces . . . . . . . . . . . 57

4.3. Illustration of Detector Probe Unit . . . . . . . . . . 59

4.4. - Betailed Illustration of New Fiber-optic Probe . . . . . . 61

4.5. Detector Response as a Function of Field Angle for

Detector Configurations Shown in Fig. 4.3. . . . . 62

4.6. Photomultiplier Tube Linearity Curve . . . . . . . . . . 63

4.7. Geometrical Configuration of Two Principal Planes in which the Scattered Light Field was Sampled...... 65

4.8. Original Configuration of Beam-forming Optics . . . . . . 67

4.9. Improved Configuration of Beam-forming Optics . . . . . . 68

4.10. Photograph of Stray Light Reaching the Observation Space . . . . . . . . . . . . . . . . . .

4.11. Illustration of Components Comprising System

Spread Function . . . . . . . . . . . . . 77

4.12. Polar Plot of Relative Intensity as a Function of

Scattering Angle . . . . . . . . . . . . 78

4.13. Illustration of Relative Intensity Plotted as a

Function of Scattering Angle . . . . . . . . . .

4.14. Illustration of BRDF Profiles Plotted as a

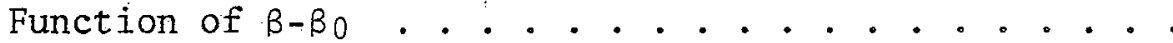


LIST OF ILLUSTRATIONS--Continued

Figure

4.15. Illustration of Scattering Function Plotted as a Function of $\beta-\beta_{O} \ldots . . . . . . . . . .884$

5.1. Comparison of Scatter Profiles for Samples with a Wide Range of Surface Roughnesses for a Normally Incident Beam .............. 86

5.2. Electron Micrographic Display Illustrating the Surface Structure of Fused Silica at Various Stages of the Grinding and Polishing Process ....... 87

5.3. Comparison of Scatter Profiles from Special Materials and Unusual Fabrication Techniques with Those of Conventional Optica1 Surfaces . . . . . . . . 89

5.4. Comparison of Scatter Profiles for Different Incident Angles ................. . . 9 90

5.5. Comparison of Scatter Profiles Taken in Different Directions for an Incident Beam at 45 Degrees

5.6. Illustration of the Importance of the Coordinate System within which the Scattering Process is Discussed

5.7. Computer Output from Inverse Scattering Program for Sample \#172 $(\lambda=0.51 .45 \mu \mathrm{m})$

5.8. Computer Output from Inverse Scattering Program for Sample \#184 $(\lambda=0.5145)$. . . . . . . . . . . 96

5.9. Surface Autocovariance Function for Sample \#198 Predicted from Scattered Light Measurements at $\lambda=0.5145 \mu \mathrm{m}$

5.10. Surface Autocovariance Function for Sample \#186 Predicted from Scattered Light Measurements at $\lambda=0.5145 \mu \mathrm{m}$................. . 98

5.11. Surface Autocovariance Function for Sample \#163

Predicted from Scattered Light Measurements at $\lambda=0.5145 \mu \mathrm{m}$ 
LIST OF ILLUSTRATIONS--Continued

Figure

Page

5.12. Surface Autocovariance Function for Sample 1NWC

Predicted from Scattered Light Measurements

at $\lambda=0.5145 \mu \mathrm{m}$. . . . . . . . . . . . . . 100

5.13. Surface Autocovariance Function for Sample 2NWC

Predicted from Scattered Light Measurements

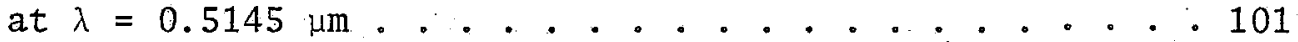

5.14. Typical Electron-Micrograph Stereo Pair of a Ground Glass

Sample along with a Surface Profile as Determined by

Stereo-Photogrammetric Techniques . . . . . . . . 103

5.15. An Electron Micrograph of Sample \#172 is Shown along with

a Surface Profile, the Surface Height Distribution, and the Surface Autocovariance Function as

Determined by Stereo-Photogrammetric Techniques . . . 105

5.16. Surface of Sample \#198 as Determined by the Scanning

FECO Interferometer at the Naval Weapons Center . . . 107

5.17. Indirect Verification of Inverse Scattering Problem . . . 110

5.18. Experimental Verification of Wavelength Scaling

Law for Smooth Surfaces . . . . . . . . . . . . . 112

5.19. Diagram I1lustrating the Effects of the

Wavelength Scaling Law . . . . . . . . . . . 113

5.20. The Angular Range of Validity for Predictions of

Scattered Light Behavior Based upon Measurements

from $1^{\circ}$ to $80^{\circ}$ at $10.6 \mu \mathrm{m}$. . . . . . . . . . . . 115

6.1. Illustration of the Phase Variation Introduced by

Reflection from a Rough Surface . . . . . . . . 119

B.1. Geometry of Incident and Reflected Elementary Beams

Used to Define the Bidirectional Reflectance

Distribution Function . . . . . . . . . . . . 128 


\section{LIST OF TABLES}

Table

Page

2.1. Tabulation of Expressions for the Aberration

Coefficients for Several Different Geometrical

Configurations . . . . . . . . . . . . . . . . 24

4.1. Surface Preparation Techniques . . . . . . . . . . . 72

5.1: Surface Characteristics . ............... . 108 
ABSTRACT

An investigation of the relationship between surface microstructure and radiant energy scattering has been conducted. Light scattering from optical surfaces is treated as a diffraction process in which the pupil function has random phase variations in addition to any existing amplitude variations. A new theory of surface scatter phenomena has been formulated by utilizing the same Fourier techniques that have proven so successful in the area of image formation. An analytical expression has been obtained for a surface transfer function which relates the surface micro-roughness to the scattered distribution of radiation from that surface: The existence of such a transfer function implies a shift-invariant scattering function which does not change shape with the angle of the incident beam. This result greatly reduces the quantity of data required to completely characterize the scattering properties of a surface. "For a large class of well-behaved surfaces this transfer function is described in terms of only the rms surface roughness and the surface autocovariance function. It thus provides a straightforward solution to the inverse scattering problem (i.e., determining surface characteristics from scattered light measurements). Once the surface characteristics are known, the same theory provides an equally simple method of predicting the wavelength dependence of the scattered light distribution. 
An extensive experimental program has accompanied this theoretical development. The apparatus and experimental procedures utilized in measuring the angular distribution of light scattered from a variety of optical surfaces for several different angles of incidence and wavelengths are described in detail. Experimental verification of the shift-invariant scattering function has been successfully demonstrated for smooth surfaces $\left(\sigma_{W}<<\lambda\right)$. The scattered light measurements from rough (diffusely reflecting) surfaces results in a scattering function which is shift-invariant over only a small range of angles and departs significantly from the predicted behavior at large scattering angles.

A computer program has been developed that operates upon scattered light data to yield the total integrated scatter, the surface transfer function, the rms surface roughness, and the surface autocovariance function. Although accurate determination of microstructure on optical surfaces is extremely difficult to accomplish by direct measurement (thus the motivation for attempting to solve the inverse scattering problem); favorable comparisons of predicted surface characteristics with the corresponding measured quantities have been demonstrated for both. smooth surfaces and moderately rough surfaces. In addition, experimental verification of the inverse scattering program was accomplished indirectly by supplying scattered light data of one wavelength as input to the inverse scattering program in order to determine the relevant surface characteristics; then this information was used to predict the scattering function at a different wavelength. 
Excellent agreement with the measured scattering function at that wavelength was achieved.

Since the above technique involves numerical computations on sampled data, an analytical expression for a wavelength scaling law is not required to determine the scattering function at any desired wavelength. However, in order to gain insight into the wavelength dependence of surface scatter phenomena, a wavelength scaling law for smooth surfaces was derived and verified. This scaling law consists of a change in the scattering angle as well as a change in the amplitude of the scattering function with changes in wavelength. It therefore provides a valuable tool for predicting the scattering behavior in certain angular regions or wavelength ranges where direct measurements are difficult to obtain. 
CHAPTER 1

INTRODUCTION

The relationship between surface micro-structure and radiant energy scattering plays an important role in many areas of technical interest. These include the trade-off between cost and performance in the fabrication of optical surfaces, design considerations for straylight rejection systems, evaluation of machined metal mirrors for highenergy laser applications, laser-radar backscatter signature programs, and a host of other applications requiring extensive scattering data. If the scattering mechanism were completely understood, surface preparation techniques or measurement programs in many of these areas could possibly be changed to obtain more favorable results.

\section{$\underline{\text { Background }}$}

If a propagating wave is incident upon a perfectly plane surface, the reflected wave is concentrated in the specular direction as determined by the well-known laws of reflection. Another idealized. surface is the perfectly diffuse reflector which scatters light according to Lambert's cosine law. A more physically realistic situation is shown in Fig. 1.1, which illustrates the optical scattering that occurs when light is reflected from a rough surface. If the surface is not too rough the reflected light consists of a specular component plus a 


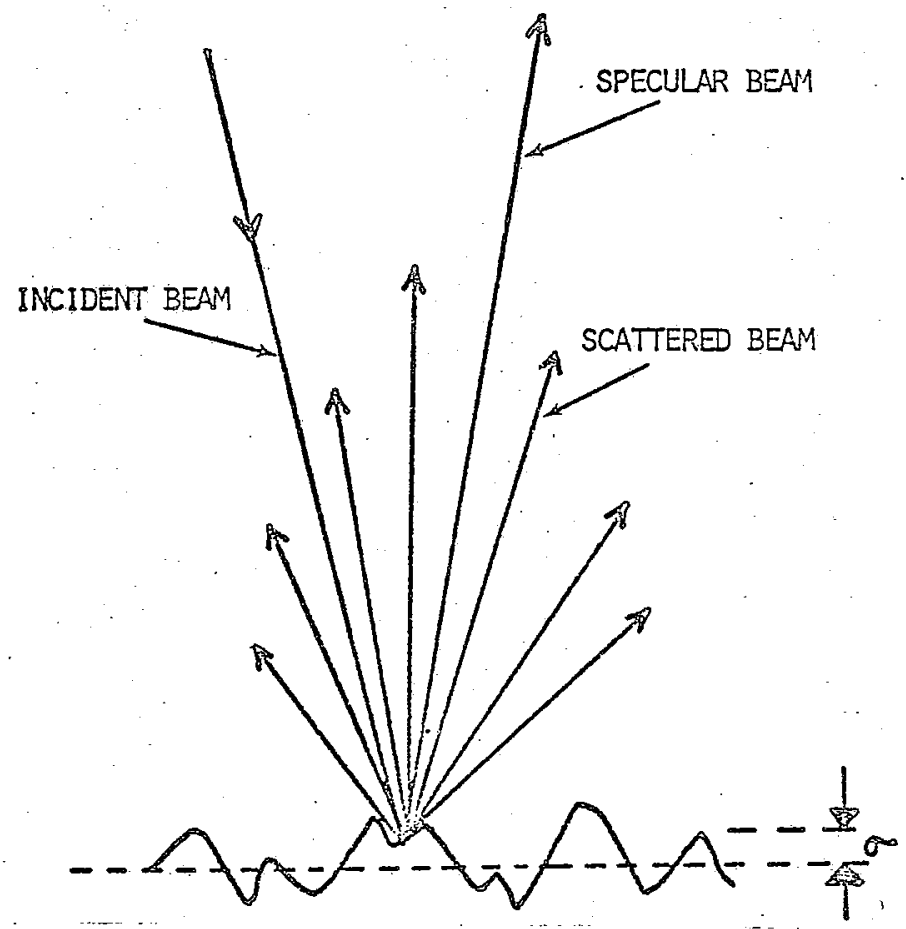

Fig. 1.1. Schematic Representation of Reflectance from a Rough Surface.

diffuse component which is scattered over a wide range of angles centered upon the specular beam.

One of the earliest investigators of scattering from a rough surface was Lord Rayleigh. In 1896 (Rayleigh, 1945) he was investigating the reflection of acoustic waves, and later (Rayleigh, 1901) he noted the effects of poorly polished surfaces on optical performance. He examined the effects of surface roughness, wavelength, and angle of incidence on the reflected beam. Chenmoganadam (1919) derived a theory of scattered 1ight based on the phase shift of the reflected beam due to the rough surface. 
However, it was not until the problem of background clutter in radar applications became apparent that a determined effort was made to solve the scattering problem for random surfaces. For example, scattering from the sea motivated the work of Davies (1954) as well as others (B1ake, 1950; Barrick, 1970; Bass, 1968; Beard, 1961; and Fuks, 1966). Considerable work has also been done in attempts to explain radar reflection from the moon (Danie1s, 1961; Evans and Pettengill, 1963; Fung and Moore, 1964; Fung, 1967; and Hagfors, 1964).

Random rough surfaces have been treated in two different ways. Rough surfaces made up of a random array of objects or shapes with known, scattering characteristics were investigated by Ament (1960), Twersky (1957), Spetner (1958), and Peake (1959). The other approach taken by Isakovich (1952), Ament (1953), Eckart (1953), Feinstein (1954), Davies (1954), and Beckmann (1957), treats the rough surface as a stochastic process.

Since optical surfaces clearly fall into the second classification of random surfaces, Bennett and Porteus (1961) expanded and experimentally investigated the scattering theory of Davies (1954). From this and subsequent. work (Bennett, 1963; and Porteus, 1963) the reflectance properties of samples with a measured surface roughness were directly compared to theory with good results. Interest in these measurements led to investigations at the Optical Sciences Center; University of Arizona, by Mott (1971), McKenney, Orme and Mott (1972), Orme (1972), DeBell and Harvey (1974), Shack and DeBel1 (1974), and Shack and Harvey (1975). 
The Bidirectional Reflectance Distribution Function (BRDF) was introduced by Nicodemus (1970) as a quantity which completely describes the reflectance (or scattering) properties of a given surface. Bidirectional reflectance data for spectral regions extending into the infrared have been collected in connection with heat-transfer analysis. Another area of interest involves the possibilities for spectrochemical analysis by reflected radiation, principally in connection with remote sensing of the earth and other planetary surfaces. Also BRDF measurements have been made on many proposed baffle materials for use in the Large Space Telescope program (Breault and Fannin, 1973). Recently there has been a great deal of activity in the area of BRDF measurements of machined metal mirrors to be used in high-energy laser applications (Young, 1975; Curcio, 1975; Decker, Bennett, and Bennett; 1975; Church and Zavada, 1975; and Stover, 1975).

\section{Dissertation Content}

In this dissertation the scattering of light from optical elements is considered to be solely a surface phenomenon. Lightscattering from optical surfaces is then treated as a diffraction process in which the pupil function has random phase variations in addition to any existing amplitude variations. A complete Fourier treatment of near-field scalar diffraction theory is therefore deve1oped in Chapter 2. This diffraction theory is generalized in Chapter 3 to include phase perturbations that lead to scattered radiation. Appropriate assumptions are then made concerning the statistical properties of optical surfaces and an analytical expression is obtained for the 
transfer function of a scattering surface. The existence of such a transfer function implies a shift-invariant scattering function which does not change shape with the angle of the incident beam. This result greatly reduces the quantity of data required to completely characterize the scattering properties of a surface. For a large class of we11behaved surfaces this transfer function is described in terms of only the rms surface roughness and the surface autocovariance function. It thus provides a straightforward solution to the inverse scattering problem (i.e., determining surface characteristics from scattered light measurements). Once the surface characteristics are known, the same theory provides an equally simple method of predicting the wavelength dependence of the scattered light distribution.

An extensive experimental program has accompanied this theoretical development. Chapter 4 describes in detail the apparatus and experimental procedures utilized in measuring the angular distribution of light scattered from a variety of optical surfaces for several different angles of incidence and wavelengths. The results of these experiments are reported in Chapter 5 and compared to theoretical predictions with generally good agreement.

Consistent with most research efforts, not all questions concerning this topic are completely answered in this dissertation. After a brief summary of results, Chapter 6 is therefore devoted to a few comments concerning new theoretical considerations and suggestions for future research. 


\section{CHAPTER 2}

\section{A FOURIER TREATMENT OF NEAR-FIELD \\ SCALAR DIFFRACTION THEORY}

The phenomenon of diffraction involves a wave field incident upon one or more objects or apertures with absorbing or conducting surfaces. The calculation of the wave field emerging from such a diffracting system is the goal of all diffraction theories.

It should be emphasized that both the Kirchhoff and RayleighSommerfeld theories, as well as the present discussion in this paper, treat light as a scalar phenomenon. (For a detailed treatment of the historical development of diffraction theory, see Goodman, 1968, pp. 30-56.) Such an approach entirely neglects the fact that the various components of the electric and magnetic field vectors are coupled through Maxwe11's equations and cannot be treated independently.

Microwave experiments have shown that scalar theory yields very accurate results provided that: (1) the diffracting aperture is large compared to a wavelength, and (2) the diffracted wave field is observed far from the aperture. It is significant that a1though the present treatment is limited by being a scalar theory, the above approximations are not imposed during the mathematical formulation as they are in the Kirchhoff theory. Furthermore, the following development provides much more insight than the conventional RayleighSommerfeld theory. 


\section{The Diffracted Wave Field \\ as a Superposition of Plane Waves}

The fundamental diffraction problem consists of two parts: (1) determining the effect of introducing the diffracting screen upon the field immediately behind the diffracting screen, and (2) determining how it affects the field downstream from the diffracting screen (i.e., what is the field immediately behind the diffracting screen and how does it propagate).

Consider first the propagation problem and let the complex amplitude distribution of the optical disturbance in plane $P_{0}$ be represented by the scalar function $U_{0}(\hat{x}, \hat{y} ; 0)$. This scalar disturbance in $P_{0}$ will be considered the only radiation contributing to the field $U(\hat{x}, \hat{y} ; \hat{z})$ in plane $P$ (see Fig. 2.1), $\hat{z}$ has a parametric relationship since it is a function of the observation plane. Note that a scaled coordinate system is utilized in which $\hat{x}=x / \lambda, \hat{y}=y / \lambda, \hat{z}=z / \lambda$.

\section{Initial Conditions.}

It will be assumed that the complex amplitude of any monochromatic optical disturbance propagating through free space must obey the time-independent wave equation (Helmholtz equation). We will also assume that the Fourier transform of the scalar field $U_{0}(\hat{x}, \hat{y} ; 0)$ exists. This is not a severe restriction, however, as Bracewell (1965) points 


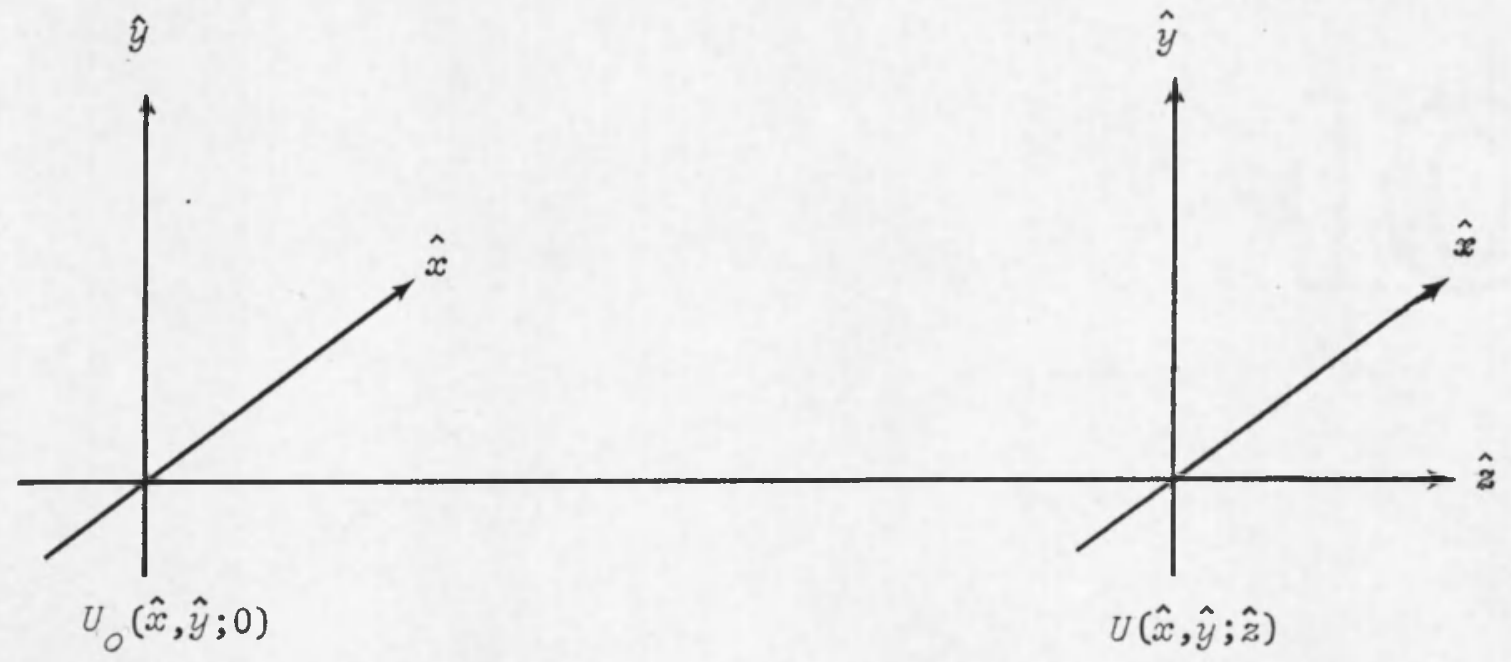

Fig. 2.1. Geometry of Planes $P_{0}$ and $P$. 
out that physical possibility is a valid sufficient condition for the existence of a Fourier transform.

The Direct Application of Fourier Transform Theory

We can thus define the following Fourier transform relationships that exist for planes $P_{0}$ and $P$.

$$
\begin{aligned}
& A_{0}(\alpha, \beta ; 0)=\iint_{-\infty}^{\infty} U_{0}(\hat{x}, \hat{y} ; 0) \mathrm{e}^{-i 2 \pi(\alpha \hat{x}+\beta \hat{y})} d \hat{x} d \hat{y} \\
& U_{0}(\hat{x}, \hat{y} ; 0)=\int_{-\infty}^{\infty} A_{0}(\alpha, \beta ; 0) \mathrm{e}^{i 2 \pi(\alpha \hat{x}+\beta \hat{y})} d \alpha d \beta \\
& A(\alpha, \beta ; \hat{z})=\int_{-\infty}^{\infty} U(\hat{x}, \hat{y} ; \hat{z}) \mathrm{e}^{-i 2 \pi(\alpha \hat{x}+\beta \hat{y})} d \hat{x} d \hat{y} \\
& U(\hat{x}, \hat{y} ; \hat{z})=\int_{-\infty}^{\infty} A(\alpha, \beta ; \hat{z}) \mathrm{e}^{i 2 \pi(\alpha \hat{x}+\beta \hat{y})} d \alpha d \beta .
\end{aligned}
$$

Equations (2) and (4) indicate that the monochromatic scalar wave field in planes $P_{0}$ and $P$ can be decomposed into plane wave components whose amplitudes are a function of the direction cosines of the propagation vector. The functions $A_{O}(\alpha, \beta ; 0)$ and $A(\alpha, \beta ; \hat{z})$ will be referred to as the direction cosine spectrum of plane waves contributing to the disturbance $U_{0}(\hat{x}, \hat{y} ; 0)$ and $U(\hat{x}, \hat{y} ; \hat{z})$ respectively. The direction 
cosine spectrum of plane waves is used here in lieu of the angular spectrum of plane waves discussed by Ratcliffe (1956) and others. This is consistent with a more general treatment which is not restricted to small angles.

In the scaled coordinate system $\hat{\nabla}^{2}=\lambda^{2} \nabla^{2}$, and $\hat{k}^{2}=\lambda^{2} k^{2}=$ $(2 \pi)^{2}$. Hence the Helmholtz equation becomes

$$
\left[\hat{\nabla}^{2}+(2 \pi)^{2}\right] U(\hat{x}, \hat{y} ; \hat{z})=0
$$

Now by applying Eq. (4) and requiring the individual plane wave components to satisfy the Helmholtz equation, we find

$$
A(\alpha, \beta ; \hat{z})=A_{O}(\alpha, \beta ; 0) \mathrm{e}^{i 2 \pi \gamma \hat{z}}
$$

where

$$
\gamma=\sqrt{1-\alpha^{2}-\beta^{2}} \text {. }
$$

The Transfer Function of Free Space

Since Eq. (6) relates the Fourier transforms of the scalar fields in planes $P_{0}$ and $P$ it can be rewritten in terms of a transfer function for free space, $H(\alpha, \beta ; \hat{z})$

$$
H(\alpha, \beta ; \hat{z}) \equiv \frac{A(\alpha, \beta ; \hat{z})}{A_{O}(\alpha, \beta ; 0)}=\mathrm{e}^{i 2 \pi \gamma \hat{z}} .
$$

We have thus far applied no restrictions on $\gamma$ and two regions of interest are apparent: that for real values of $\gamma$ and that for imaginary values, 


$$
\gamma=\sqrt{1-\left(\alpha^{2}+\beta^{2}\right)}\left\{\begin{array}{l}
\text { for }\left(\alpha^{2}+\beta^{2}\right) \leq 1 \quad \gamma \text { is real } \\
\text { for }\left(\alpha^{2}+\beta^{2}\right)>1 \quad \gamma \text { is imaginary. }
\end{array}\right.
$$

Consider now a unit circle in the $\alpha-\beta$ plane of direction cosine space as shown in Fig. 2.2. Inside this unit circle $\gamma$ is real and the corresponding part of the disturbance will propagate and contribute to the wave field in plane $P$. However, those components of the direction cosine spectrum which lie outside the unit circle have imaginary values of $\gamma$ and represent that part of the disturbance which experiences a rapid exponential decay. This is the part of the disturbance which is commonly referred to as the evanescent wave (Goodman, 1968).

Let $U_{0}(\hat{x}, \hat{y} ; 0)$ be the product of the complex amplitude transmittance of a diffracting screen and the complex amplitude distribution incident upon it. Figure 2.3(a) illustrates this quantity broken down into the part which propagates and the part which makes up the evanescent wave for the case of a unit amplitude plane wave normally incident upon a circular aperture. The direction cosine spectrum of plane waves associated with these respective optical disturbances are shown in Fig. $2.3(\mathrm{~b})$.

Note that the sharp corners on the original disturbance in Fig. 2.3(a) correspond to Kirchhoff's unnecessary boundary conditions. It is the propagating part only that accurately represents the 
12

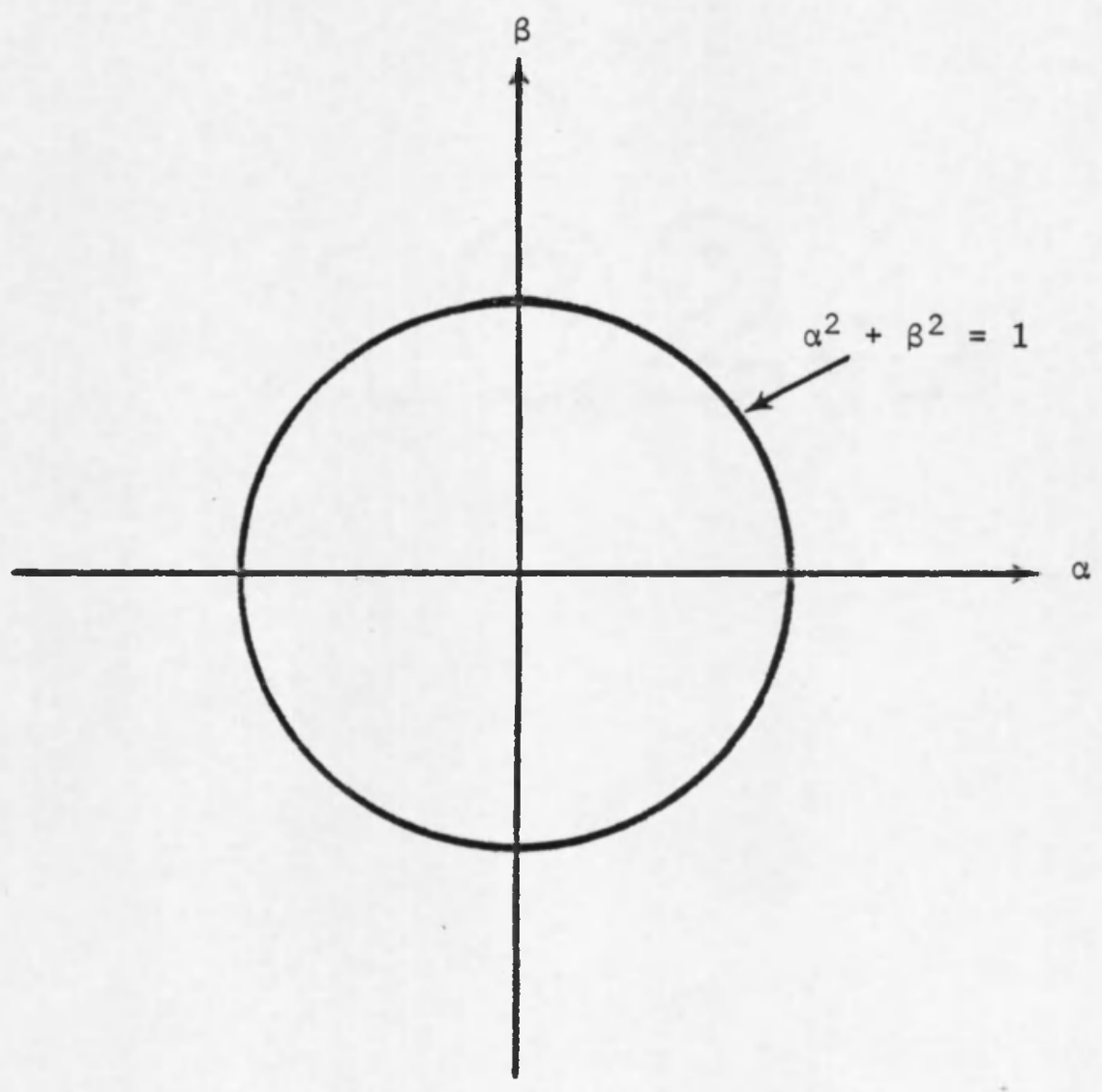

Fig. 2.2. Unit Circle in Direction Cosine Space.

The plane wave components inside this circle will propagate, and the plane wave components outside this circle contribute to the evanescent wave. 


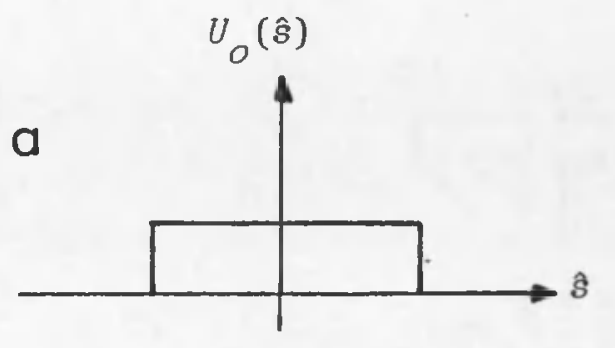

Original disturbance
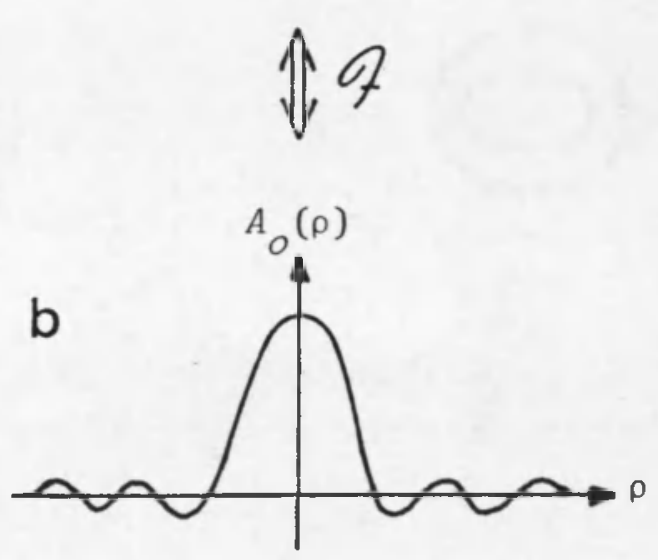

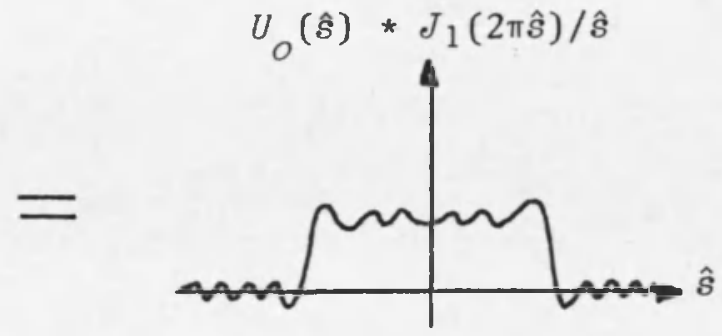

Propagating part
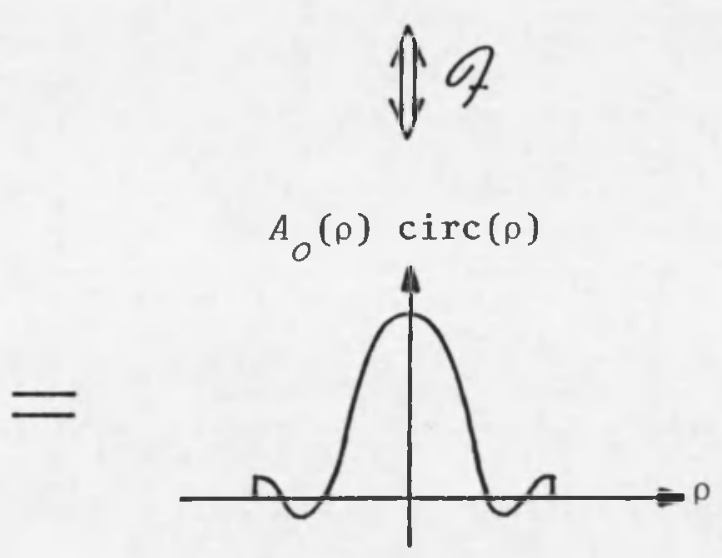

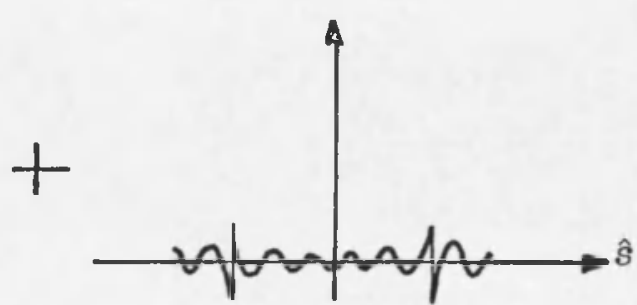

Evanescent part
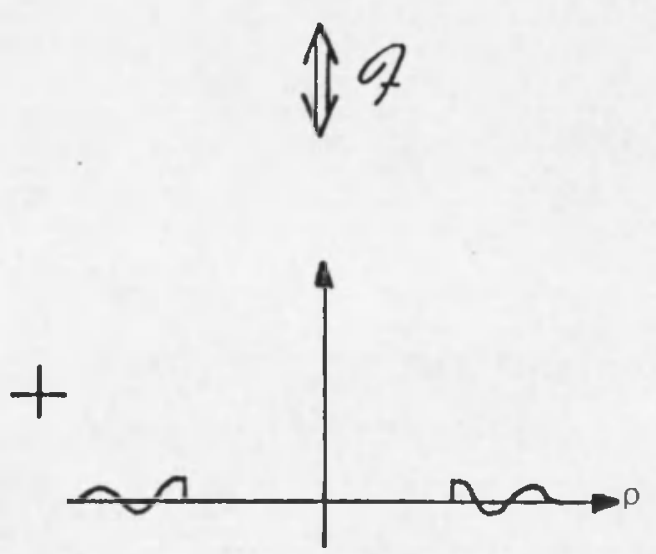

Fig. 2.3. Illustration of the Propagating and Evanescent Parts of the Diffracted Wave Field.

(a) Original disturbance separated into its propagating and evanescent parts.

(b) Direction cosine spectrum separated into its propagating and evanescent parts. 
disturbance immediately behind the diffracting aperture which will contribute to the disturbance downstream.

It is now clear that the complex amplitude distribution in plane $P$ can be determined by Fourier transforming the original disturbance $U_{0}(\hat{x}, \hat{y} ; 0)$, then multiplying the resulting direction cosine spectrum of plane waves $A_{0}(\alpha, \beta ; 0)$ by the transfer function of free space given in Eq. (7), and finally by applying the inverse Fourier transform integral of Eq. (4). However, the limits of integration on Eq. (4) must be changed such that the integration is performed only over the unit circle instead of over the entire $\alpha-\beta$ plane.

The above analysis, in which an optical disturbance is represented as a superposition of plane waves, corresponds to the transfer function approach in image formation and yields considerable insight into the behavior of these plane wave components during the phenomenon of diffraction.

The Diffracted Wave Field as a superposition of Spherical Waves

The convolution theorem (Bracewe11, 1965) of Fourier transform theory requires that a convolution operation exists in the domain of real space that is equivalent to Eq. (6).

The Point Spread Function

We thus have the alternative method of expressing the complex amplitude distribution in the observation plane by the convolution of 
the original disturbance with a point spread function. The point spread function is obtained by taking the inverse Fourier transform of the transfer function found in Eq. (7).

Starting with the well-known Weyl expansion formula (Weyl, 1919), Lalor (1968) obtained a result which, with straightforward modification, yields

$$
\int_{-\infty}^{\infty} \int^{i 2 \pi \gamma \hat{z}} e^{i 2 \pi(\alpha \hat{x}+\beta \hat{y})} d \alpha d \beta=-\frac{1}{2 \pi} \frac{\partial}{\partial \hat{z}}\left[\frac{e^{i 2 \pi \hat{r}}}{\hat{r}}\right],
$$

where

$$
\hat{r}^{2}=\hat{x}^{2}+\hat{y}^{2}+\hat{z}^{2} \text {. }
$$

The left side of Eq. (9) is the inverse Fourier transform of the transfer function of free space. The appropriate point spread function is thus given by

$$
h(\hat{x}, \hat{y} ; \hat{z})=\bar{F}^{-1}\left\{e^{i 2 \pi \gamma \hat{z}}\right\}=\left(\frac{1}{2 \pi \hat{r}}-i\right) \frac{\hat{z}}{\hat{x}} \frac{e^{i 2 \pi \hat{r}}}{\hat{r}} .
$$

Huygens' Principle

Recall now the assertion by Christiaan Huygens (Thompson, 1912) in 1678 that each element of a wavefront may be regarded as the center of a secondary disturbance which gives rise to spherical wavelets; and moreover that the position of the wavefront at any later time is the envelope of all such wavelets. These intuitive convictions, sometimes called Huygens' wavefront construction, are an excellent description of 
a convolution operation in which the initial disturbance is convolved with a Huygens' wavelet. It is therefore quite appropriate to think of the point spread function of a diffraction system as the intersection of a Huygens' wavelet with the observation plane.

Equation (10) is therefore an exact mathematical expression for a Huygens' wavelet which is valid right down to the initial disturbance itse1f. However, for $\hat{r} \gg 1$,

$$
h(\hat{x}, \hat{y} ; \hat{z})=-i(\hat{z} / \hat{r}) \frac{e^{i 2 \pi \hat{r}}}{\hat{r}}=(\hat{z} / \hat{r}) \frac{e^{i 2 \pi(\hat{r}-1 / 4)}}{\hat{r}},
$$

it reduces to the familiar expression for a spherical wave with cosine obliquity factor and a $\pi / 2$ phase delay.

General Rayleigh-Sommerfeld Diffraction Formula

If we write down the convolution integral for the disturbance in the observation plane, using the expression in Eq. (10) for $h(\hat{x}, \hat{y} ; \hat{z})$, we obtain the general Rayleigh-Sommerfeld diffraction formula

$$
U(\hat{x}, \hat{y} ; \hat{z})=\iint_{-\infty}^{\infty} U_{0}\left(\hat{x}^{\prime}, \hat{y}^{\prime} ; 0\right)\left(\frac{1}{2 \pi \hat{l}}-i\right) \hat{z} / \hat{l} \frac{e^{i 2 \pi \hat{l}}}{\hat{l}} d \hat{x}^{\prime} d \hat{y}^{\prime}
$$

where

$$
\hat{l}^{2}=\left(\hat{x}-\hat{x}^{\prime}\right)^{2}+\left(\hat{y}-\hat{y}^{\prime}\right)^{2}+\hat{z}^{2} \text {. }
$$

This is an exact expression for the diffracted wave field which is valid throughout the entire space in which the diffraction occurs--right down 
to the aperture. No approximations have been made in this scalar theory. Furthermore, the above equation expresses the disturbance on the observation plane as a superposition of spherical waves which corresponds to the spread function approach in image formation.

\section{Geometrical Configurations of the Observation Space}

In order to insure a space invariant point spread function our equations have been restricted to mapping an optical disturbance from an input plane to an output plane, where $\hat{z}$ has a parametric relationship since its value determines the location of the output plane. However, the summation of these Huygens' wavelets is valid over any surface. The above treatment thus gives us a far more powerful conceptual tool than provided by the equations themselves.

We will therefore investigate the properties of the diffracted wave field on two particular geometrical configurations of the observation surface.

The Diffracted Wave Field on a Plane

Equation (12) reduces to the more familiar but less general form of the Rayleigh-Sommerfeld diffraction formula when $\hat{z} \gg 1$,

$$
U(\hat{x}, \hat{y}, \hat{z})=-i \iint_{-\infty}^{\infty} U_{0}\left(\hat{x}^{\prime}, \hat{y}^{\prime} ; 0\right) \frac{\hat{z}}{\hat{\ell}} \frac{e^{i 2 \pi \hat{l}}}{\hat{l}} d \hat{x}^{\prime} d \hat{y}^{\prime} .
$$

The following algebraic substitutions

$$
\hat{\ell}=\hat{z}(1+\delta), \quad \delta=\frac{\hat{\ell}-\hat{z}}{\hat{z}}
$$


allow us to rewrite Eq. (14) as

$$
U(\hat{x}, \hat{y} ; \hat{z})=\frac{e^{i 2 \pi \hat{z}}}{i \hat{z}} \int_{-\infty}^{\infty} U_{0}\left(\hat{x}^{\prime}, \hat{y}^{\prime} ; 0\right) \frac{1}{(1+\delta)^{2}} e^{i 2 \pi(\hat{l}-\hat{z})} d \hat{x}^{\prime} d \hat{y}^{\prime} .
$$

Note that we have imposed no restriction upon the size of the aperture or the size of the observation space. The only limitation on the above equation is that the observation plane must be many wavelengths from the aperture.

The above diffraction formula is a rather unwieldy integral to solve explicitly for most problems of practical interest. The Fresnel and Fraunhofer diffraction formulas are obtained by retaining only the first two terms in the binomial expansion for the quantity

$$
l=\hat{z}\left(1+\left[\left(\hat{x}^{2}+\hat{y}^{2}\right) / \hat{z}^{2}+\left(\hat{x}^{\prime 2}+\hat{y}^{\prime 2}\right) / \hat{z}^{2}-2\left(\hat{x} \hat{x}^{\prime}+\hat{y} \hat{y}^{\prime}\right) / \hat{z}^{2}\right]\right)^{\frac{1}{2}} .
$$

However, severe restrictions are then imposed upon the size of the aperture and the region over which the calculations are valid in the observation plane. In order that we do not impose these restrictions, all terms from the binomial expansion must be retained. This can be accomplished by rewriting Eq. (15) as the following Fourier transform integral

$$
U(\hat{x}, \hat{y} ; \hat{z})=\frac{e^{i 2 \pi \hat{z}}}{i \hat{z}} \iint_{-\infty}^{\infty} \boldsymbol{U}_{0}\left(\hat{x}^{\prime}, \hat{y}^{\prime} ; \hat{x}, \hat{y}\right) \mathrm{e}^{-\frac{i 2 \pi}{\hat{z}}\left(\hat{x}^{\prime} \hat{x}+\hat{y}^{\prime} \hat{y}\right)} d \hat{x}^{\prime} d \hat{y}^{\prime},
$$

where the complex quantity 


$$
\mathscr{U}_{0}\left(\hat{x}^{\prime}, \hat{y}^{\prime} ; \hat{x}, \hat{y}\right)=T_{0}\left(\hat{x}^{\prime}, \hat{y}^{\prime} ; 0\right) \frac{1}{(1+\delta)^{2}} e^{i 2 \pi \hat{W}}
$$

can be regarded as a generalized pupil function. $T_{0}(\hat{x}, \hat{y} ; 0)$ is the complex amplitude transmittance of the diffracting aperture (or aperture function), and all of the terms from the binomial expansion for the quantity $(\hat{\ell}-\hat{z})$, except for the term which was extracted for use as the Fourier kernel, are lumped together in the quantity $\hat{W}$ along with any phase variations in the incident wavefront.

Equation (16) clearly reduces to the conventional Fresnel diffraction formula when a plane wave is incident upon the aperture and when $\hat{z}$ is sufficiently large such that $\hat{\ell}$ is adequately approximated by retaining only the first two terms of the binomial expansion.

The Diffracted Wave Field on a Hemisphere

Let us now examine the diffracted wave field on a hemisphere centered upon the diffracting aperture as illustrated in Fig. 2.4. The position of an arbitrary observation point will be specified by the direction cosines $\alpha$ and $\beta$ of its position vector, and the radius $\hat{p}$ of the hemisphere upon which it resides. Note that

$$
\alpha=\hat{x} / \hat{r}, \quad \beta=\hat{y} / \hat{r} \text {, and } \gamma=\hat{z} / \hat{r} \text {, }
$$

where

$$
\hat{p}^{2}=\hat{x}^{2}+\hat{y}^{2}+\hat{z}^{2} \text {. }
$$

The following algebraic substitutions

$$
\hat{l}=\hat{r}(1+\varepsilon) ; \quad \varepsilon=(\hat{l}-\hat{r}) / \hat{r}
$$

allow us to rewrite the general Rayleigh-Sommerfeld diffraction formula 


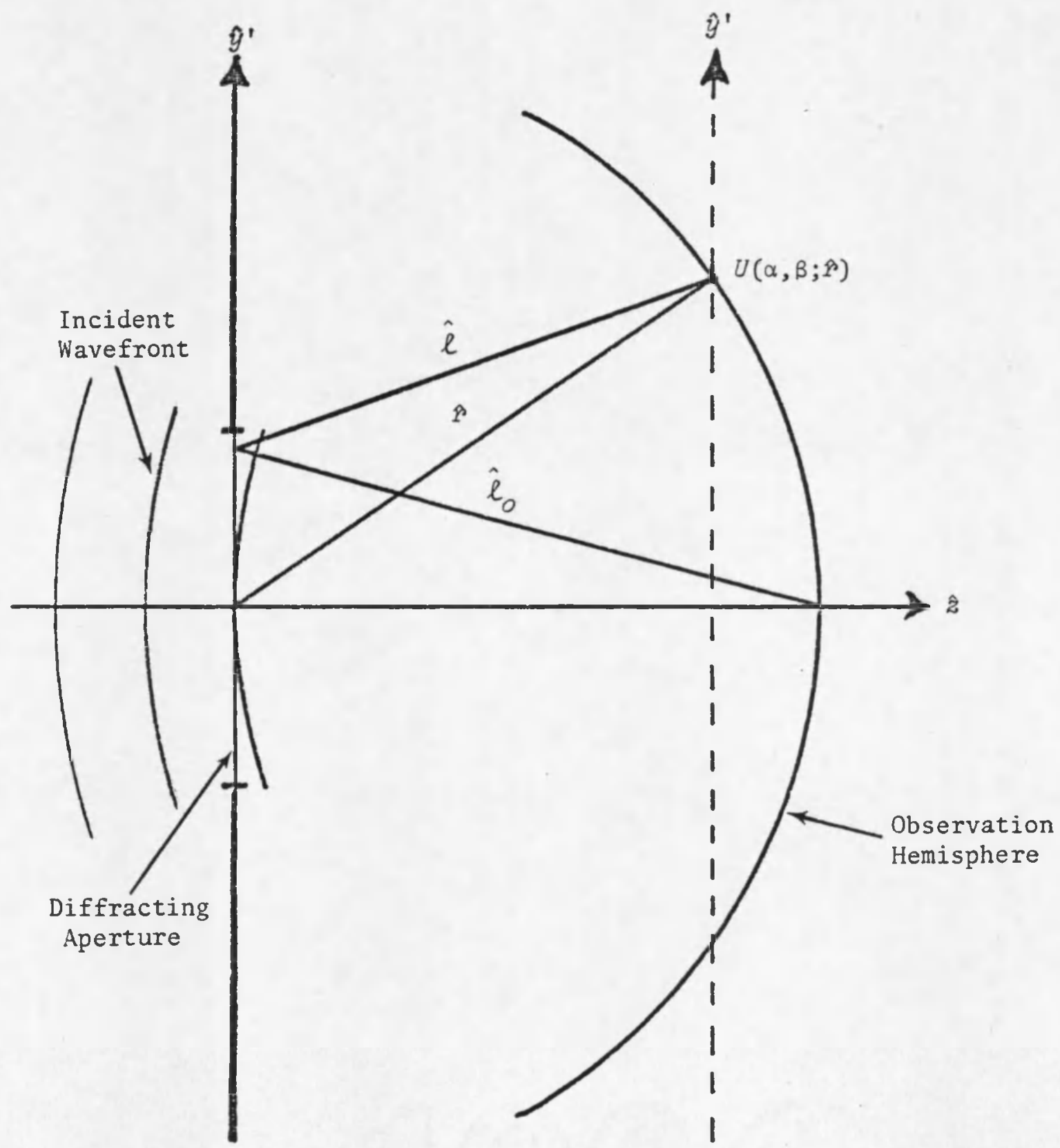

Fig. 2.4. Geometrical Relationship Between Incident Beam, Diffracting Aperture, and Observation Hemisphere. 
expressed in Eq. (12) as

$$
\begin{aligned}
U(\alpha, \beta ; \hat{r}) & =\gamma \frac{e^{i 2 \pi \hat{r}}}{\hat{r}} \int_{-\infty}^{\infty} U_{0}\left(\hat{x}^{\prime}, \hat{y}^{\prime} ; 0\right) \\
& \times\left[\frac{1}{2 \pi \hat{r}(1+\varepsilon)}-i\right] \frac{1}{(1+\varepsilon)^{2}} \mathrm{e}^{i 2 \pi(\hat{\ell}-\hat{r})} d \hat{x}^{\prime} d \hat{y}^{\prime} .
\end{aligned}
$$

We now have an exact expression for the diffracted wave field on an observation hemisphere which is valid throughout the entire half-space behind the plane of the diffracting aperture.

If we now require that $\hat{r} \gg 1$ and make the appropriate binomial expansion for the quantity $(\hat{\ell}-\hat{r})$, we again obtain a Fourier transform integral

$$
U(\alpha, \beta ; \hat{r})=\gamma \frac{e^{i 2 \pi \hat{r}}}{i \hat{r}} \int_{-\infty}^{\infty} \int_{0}\left(\hat{x}^{\prime}, \hat{y}^{\prime} ; \alpha, \beta\right) \mathrm{e}^{-i 2 \pi\left(\alpha x^{\prime}+\beta y^{\prime}\right)} d \hat{x}^{\prime} d \hat{y}^{\prime},
$$

where the generalized pupil function is given by

$$
\mathscr{U}_{0}\left(\hat{x}^{\prime}, \hat{y}^{\prime} ; \alpha, \beta\right)=T_{0}\left(\hat{x}^{\prime}, \hat{y}^{\prime} ; 0\right) \frac{1}{(1+\varepsilon)^{2}} e^{i 2 \pi \hat{W}} .
$$

Once again, all of the terms from the binomial expansion for the quantity $(\hat{\ell}-\hat{s})$, except for the term which was extracted for use as the Fourier kernel, are lumped together in the quantity $\hat{W}$ along with any phase variations in the incident wavefront. 


\section{Aberrations of Diffracting Systems}

The quantity $\hat{W}$ in Eqs. (17) and (23) represent phase variations in the diffracted wavefront emerging from the aperture. Therefore, $\hat{W}$ can be interpreted as a conventional wavefront aberration function (Hopkins, 1950) which is conveniently expressed as a general power series expansion of the pupil coordinates and the appropriate field parameters .

For the case of a rotationally-symmetric diffracting aperture we can, without loss of generality, choose the observation point to lie on the $\hat{y}$-axis $(\hat{x}=0)$. The wavefront aberration function can then be written as

$$
\begin{aligned}
& \hat{W}=\hat{W}_{200 \rho^{2}}+\hat{W}_{020} \hat{a}^{2}+\hat{W}_{111 \rho} \hat{a} \cos \phi \\
& +\hat{W}_{400 \rho^{4}}+\hat{W}_{040} \hat{a}^{4}+\hat{W}_{131} \rho \hat{a}^{3} \cos \phi+\hat{W}_{222} \rho^{2} \hat{a}^{2} \cos ^{2} \phi \\
& +\hat{W}_{220 \rho^{2}} \hat{a}^{2}+\hat{W}_{311 \rho^{3} \hat{a} \cos \phi} \\
& \text { + higher-order terms, }
\end{aligned}
$$

where $\rho$ is a normalized field position of the observation point and $\hat{a}$ is a normalized pupil height.

By equating coefficients of the corresponding terms in the appropriate binomial expansions and the above wavefront aberration function, we obtain expressions for the aberration coefficients in terms of the aperture diameter, the observation distance, and the appropriate field parameter. These aberrations, which are inherently associated with the diffraction process, are precisely the effects ignored when making the usual Fresnel and Fraunhofer approximations. Furthermore, these 
aberrations have the same functional form as the familiar aberrations caused by the refraction or reflection process in imperfect imaging systems.

The expressions for these aberration coefficients are derived in Appendix A and tabulated in Table 2.1 to enable easy comparison of several different geometrical configurations of the incident wavefront and the observation space.

Consider first a plane wave illuminating the diffracting aperture and a plane observation space. : We see from the first column of Table 2.1 that al1 aberrations are present except for lateral magnification error $\left(\hat{W}_{111}\right)$ which is absent for all geometrical configurations because this term of the binomial expansion for $\hat{l}$ is extracted for use as the kernel of the Fourier transform integral. It is clear that very large observation distances are required to reduce defocus $\left(\hat{W}_{020}\right)$ to a negligible value. Also, distortion $\left(\hat{W}_{311}\right)$ imposes severe restraints upon the field angle over which the diffracted wave field is accurately described by the Fourier transform of the aperture function. These restrictions are the same as those usually imposed during the development of the Kirchhoff theory and in most applications of the RayleighSommerfeld diffraction theory.

The effect of illuminating the aperture with a spherical wave converging to the observation plane is to eliminate defocus $\left(\hat{W}_{020}\right)$ and a11 orders of spherical aberration $\left(\hat{W}_{040}\right)$. This removes the requirement for an extremely: large observation distance, but the Fourier 
Table 2.1. Tabulation of Expressions for the Aberration Coefficients for Several Different Geometrical Configurations.

(a) Incident wavefront.

(b) Observation space.

\begin{tabular}{|c|c|c|c|c|}
\hline & $\begin{array}{l}\text { a. Plane } \\
\text { b. Plane }\end{array}$ & $\begin{array}{l}\text { a. Sphere } \\
\text { b. Plane }\end{array}$ & $\begin{array}{l}\text { a. Plane } \\
\text { b. Hemisphere }\end{array}$ & $\begin{array}{l}\text { a. Sphere } \\
\text { b. Hemisphere }\end{array}$ \\
\hline $\begin{array}{l}\text { Piston Error } \\
\hat{W}_{200}\end{array}$ & $\frac{\hat{z}}{2}\left(\frac{\hat{y}_{\max }}{\hat{z}}\right)^{2}$ & $\frac{\hat{z}}{2}\left(\frac{\hat{y}_{\max }}{\hat{z}}\right)$ & 0 & 0 \\
\hline $\begin{array}{l}\text { Defocus } \\
\hat{W}_{020}\end{array}$ & $\frac{\hat{z}}{2}\left(\frac{\hat{d}}{2 \hat{z}}\right)^{2}$ & 0 & $\frac{\hat{r}}{2}\left(\frac{\hat{d}}{2 \hat{r}}\right)^{2}$ & 0 \\
\hline $\begin{array}{l}\text { Lateral Magni- } \\
\text { fication Error } \\
\hat{W}_{111}\end{array}$ & 0 & 0 & 0 & 0 \\
\hline $\begin{array}{l}\text { 3rd-Order } \\
\text { Piston Error } \\
\hat{W}_{400}\end{array}$ & $-\frac{\hat{z}}{8}\left(\frac{\hat{y}_{\max }}{\hat{z}}\right)^{4}$ & $-\frac{\hat{z}}{8}\left(\frac{\hat{y}_{\max }}{\hat{z}}\right)$ & 0 & 0 \\
\hline $\begin{array}{l}\text { Spherical } \\
\text { Aberration } \\
\hat{W}_{040}\end{array}$ & $-\frac{\hat{z}}{8}\left(\frac{\hat{d}}{2 \hat{z}}\right)^{4}$ & 0 & $-\frac{\hat{r}}{8}\left(\frac{\hat{d}}{2 \hat{r}}\right)^{4}$ & 0. \\
\hline $\begin{array}{l}\text { Coma } \\
\hat{W}_{131}\end{array}$ & $\frac{\hat{z}}{2} \frac{\hat{y}_{\max }}{\hat{z}}\left(\frac{\hat{d}}{2 \hat{z}}\right)^{3}$ & $\frac{\hat{z}}{2} \frac{\hat{y}_{\max }}{\hat{z}}\left(\frac{\hat{z}}{2 \hat{z}}\right)^{3}$ & $\frac{\hat{r}}{2} B_{\max }\left(\frac{\hat{d}}{2 \hat{r}}\right)^{3}$ & $\frac{\hat{r}}{2} B_{\max }\left(\frac{\hat{a}}{2 \dot{i}}\right)^{3}$ \\
\hline $\begin{array}{l}\text { Astigmatism } \\
\hat{W}_{222}\end{array}$ & $-\frac{\hat{z}}{2}\left(\frac{\hat{y}_{\max }}{\hat{z}}\right)^{2}\left(\frac{\hat{d}}{2 \hat{z}}\right)^{2}$ & $-\frac{\hat{z}}{2}\left(\frac{\hat{y}_{\max }}{\hat{z}}\right)^{2}\left(\frac{\hat{d}}{2 \hat{z}}\right)^{2}$ & $-\frac{\hat{r}}{2} \beta_{\max }{ }^{2}\left(\frac{\hat{d}}{2 \hat{r}}\right)^{2}$ & $-\frac{\dot{\hat{r}}}{2} \beta_{\max }{ }^{2}\left(\frac{\hat{d}}{2 \hat{r}}\right)^{2}$ \\
\hline $\begin{array}{l}\text { Field } \\
\text { Curvature } \\
\hat{W}_{220}\end{array}$ & $-\frac{\hat{z}}{4}\left(\frac{\hat{y}_{\max }}{\hat{z}}\right)^{2}\left(\frac{\hat{d}}{2 \hat{z}}\right)^{2}$ & $-\frac{\hat{z}}{4}\left(\frac{\hat{y}_{\max }}{\hat{z}}\right)^{2}\left(\frac{\hat{z}}{2 \hat{z}}\right)^{2}$ & 0 & 0 \\
\hline $\begin{array}{l}\text { Distortion } \\
\hat{\mathrm{W}}_{311}\end{array}$ & $\frac{\hat{z}}{2}\left(\frac{\hat{y}_{\max }}{\hat{z}}\right)^{3} \frac{\hat{d}}{2 \hat{z}}$ & $\frac{\hat{z}}{2}\left(\frac{\hat{y}_{\max }}{\hat{z}}\right)^{3} \frac{\hat{d}}{2 \hat{z}}$ & 0 & 0 \\
\hline
\end{tabular}


transform of the aperture function is still valid only over a small region about the optical axis in the observation plane.

Choosing the observation space to be a hemisphere centered upon the diffracting aperture eliminates field curvature $\left(\hat{W}_{220}\right)$, distortion $\left(\hat{W}_{311}\right)$, and all orders of piston error $\left(\hat{W}_{200}\right.$ and $\left.\hat{W}_{400}\right)$.

Hence for the case of a spherical incident wave converging to the intersection of the optical axis and an observation hemisphere, only coma $\left(\hat{W}_{131}\right)$ and astigmatism $\left(\hat{W}_{222}\right)$ are present. And the values of the aberration coefficients can be calculated from the relationships provided in the last column of Table 2.1.

Thus, for a system with an aperture diameter of $1 \mathrm{~mm}$ and an observation hemisphere with a radius of $1 \mathrm{~m}$, we have for $\lambda=0.5 \mu \mathrm{m}$ and $\beta_{\max }=1$

$$
\begin{aligned}
& \hat{W}_{131}=1.25 \times 10^{-4} \\
& \hat{W}_{222}=-2.50 \times 10^{-1} .
\end{aligned}
$$

Hence there is only $\lambda / 4$ of astigmatism at the edge of the field (i.e., $90^{\circ}$ field angle).

Similarly for an $f / 10$ system $(\hat{d} / \hat{r}=0.1)$ with an aperture diameter of $5 \mathrm{~cm}$ and a maximum field angle of $0.5^{\circ}\left(\beta_{\max }=0.00872\right)$, we have for $\lambda=0.5 \mu \mathrm{m}$,

$$
\begin{aligned}
& \hat{W}_{131}=0.545 \\
& \hat{W}_{222}=-0.095 .
\end{aligned}
$$

Hence coma dominates at the edge of the field with a value of approximately $\lambda / 2$. 
Fina11y, for an $f / 10$ system with an aperture diameter of $1 \mathrm{~cm}$ and a field size equal to the size of the aperture (i.e., a pair of f/10 relay lenses $1 \mathrm{~cm}$ in diameter), we have for $\lambda=0.5 \mu \mathrm{m}$,

$$
\begin{aligned}
& \hat{W}_{1.31}=0.625 \\
& \hat{W}_{2.22}=-0.625 .
\end{aligned}
$$

We find slightly more than $\lambda / 2$ of coma and astigmatism at the edge of the field.

It should be pointed out that in each of the above cases the radius of the Airy disc in direction cosine space is approximately equal to $0.001 \beta_{\max }$. Hence the off-axis aberrations are of little more than academic interest unless there is some structure in the aperture with high spatial frequency content which will diffract light at large angles from the direction of the incident beam.

However, the above analysis of the aberrations associated with the diffraction process can be readily applied to holographic systems or systems containing diffraction gratings.

For example, an f/6 system with a 10-1ine-per-mm Ronchi ruling placed in a $40-\mathrm{mm}$ diameter aperture produces the diffraction pattern shown in Fig. 2.5. The diffracted order at three different field positions was photographed through a microscope with the following results: at $\beta=0$ no aberrations were apparent; at $\beta=0.04$ coma was predominant with a value of approximately $5 \lambda$; and at $\beta=0.10$ coma and astigmatism both have values of approximately 15 $\lambda$. These values were estimated by visual inspection of the aberrated diffracted orders. 


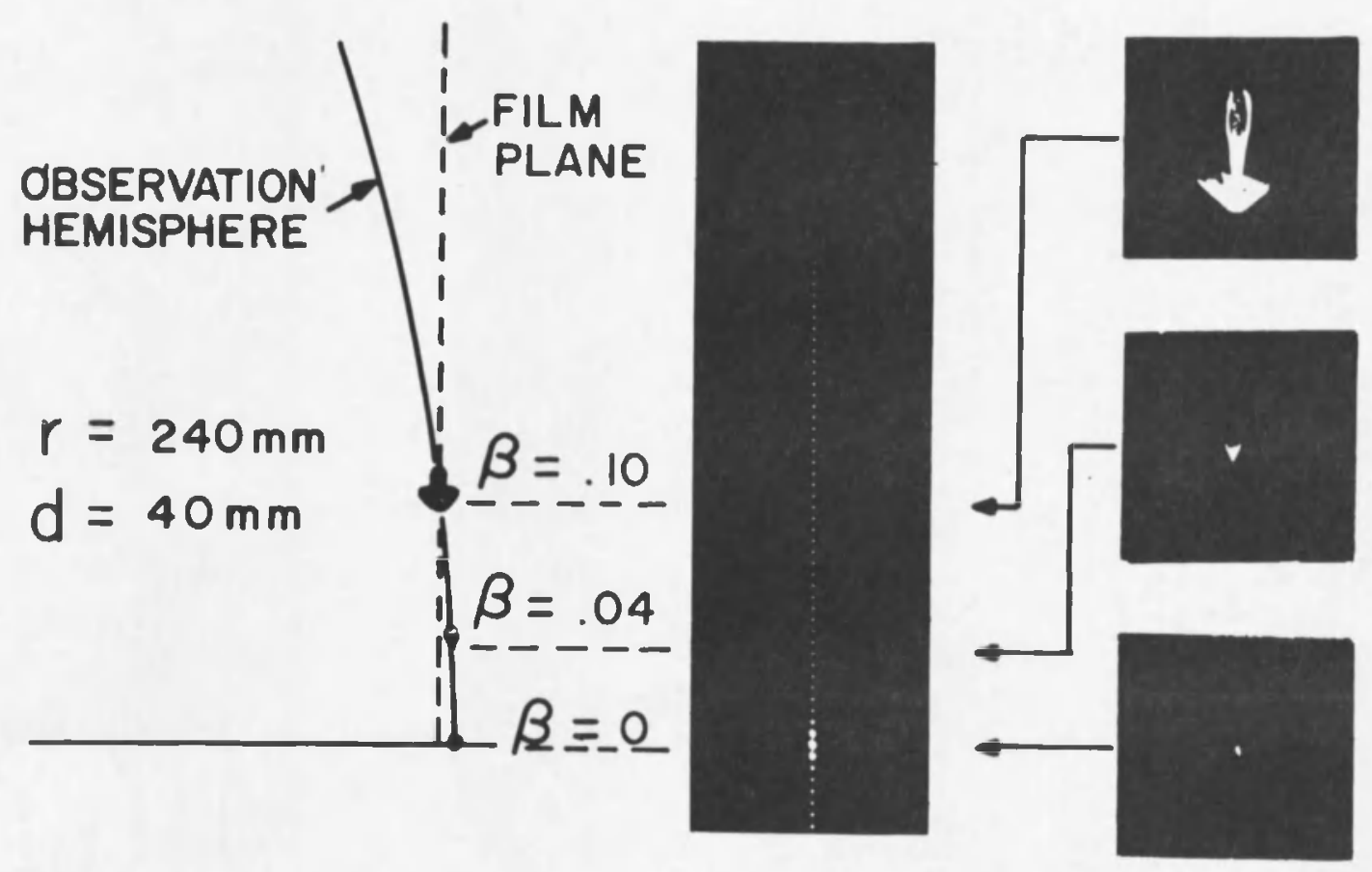

Fig. 2.5. Diffraction Pattern of a 10-Line per mm Ronchi Ruling Placed in an $\mathrm{f} / 6$ Cone of Light with a $40-\mathrm{mm}$ Diameter.

Magnified images of diffracted orders at various field positions indicate that coma is predominant for small field angles with astigmatism also becoming significant at larger field angles. 
By stopping the aperture down to a diameter of $12 \mathrm{~mm}(\mathrm{f} / 20$ system) and observing the diffracted orders at larger field angles, astigmatism becomes the predominant aberration as shown in Fig. 2.6. The sagittal focus lies on the observation hemisphere of radius $r$ and the medial and tangential surfaces have smaller radii as indicated. The diffracted order at $\beta=0.020$ exhibits about $6 \lambda$ of astigmatism. This order was observed through focus with the microscope and the magnified images are displayed.

In both of the above examples the observed aberrations are in good agreement with those predicted from the coefficients presented in Table 2.1.

\section{Shift Invariance of the Diffracted Wave Field}

We have shown that any departures of the actual diffracted wave field from that predicted by the simple Fourier transform of the aperture function take the form of conventional wavefront aberrations.

If we neglect these aberrations, Eq. (22) reduces to

$$
U(\alpha, \beta ; \hat{x})=\gamma \frac{e^{i 2 \pi \hat{x}}}{i \hat{x}} \int_{-\infty}^{\infty} \int_{0}\left(\hat{x}^{\prime}, \hat{y}^{\prime} ; 0\right) \mathrm{e}^{-i 2 \pi\left(\alpha \hat{x}^{\prime}+\beta \hat{y}^{\prime}\right)} d \hat{x}^{\prime} d \hat{y}^{\prime} .
$$

But this is merely the Fourier transform of the aperture function multiplied by a spherical Huygens' wavelet.

$$
U(\alpha, \beta ; \hat{r})=\gamma \frac{e^{i 2 \pi \hat{r}}}{i \hat{r}}\left\{T_{0}(\hat{x}, \hat{y} ; 0)\right\} .
$$



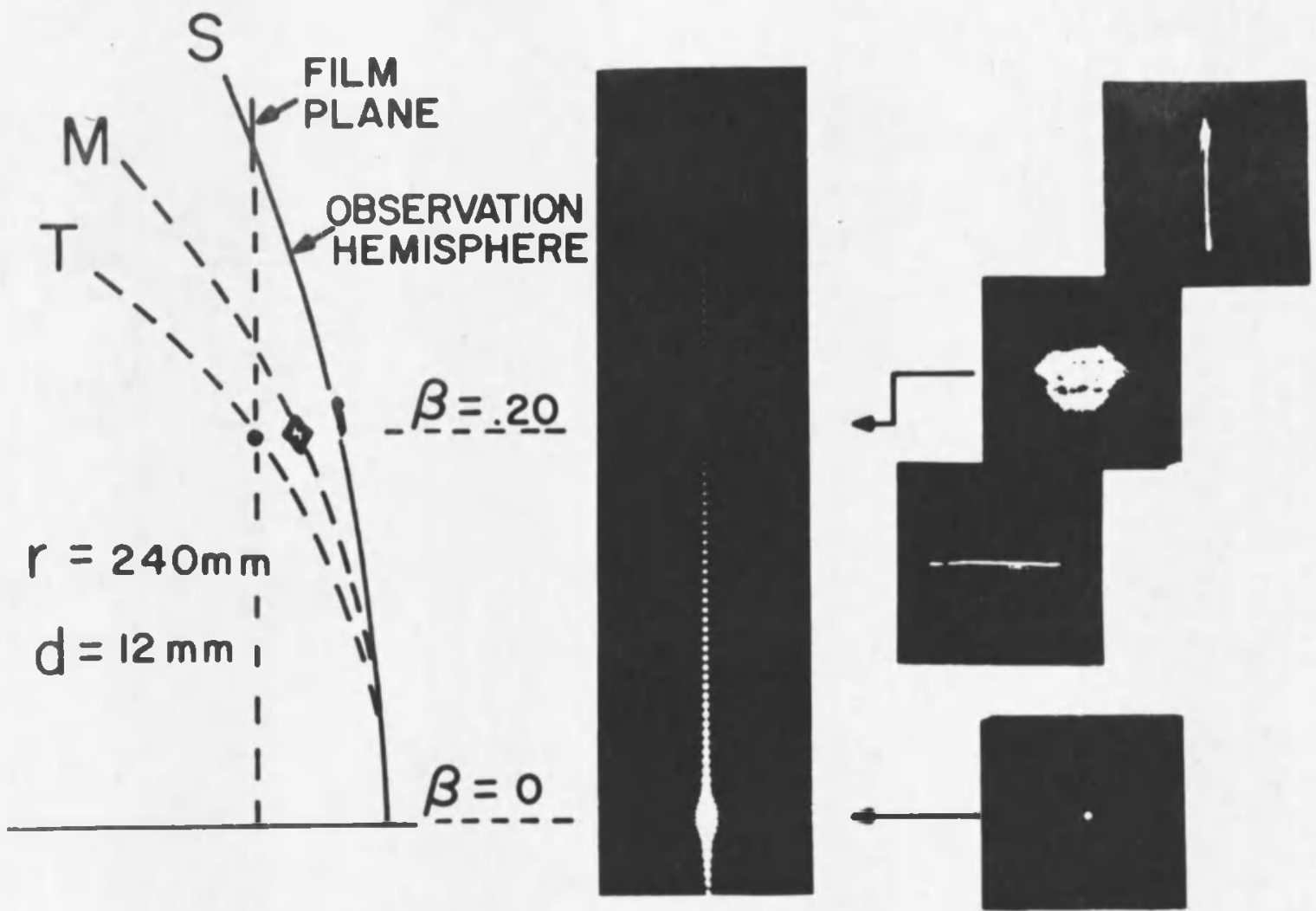

Fig. 2.6. Diffraction Pattern of a 10-Line per mm Ronchi Ruling Placed in an $f / 20$ Cone of Light with a 12-mm Diameter.

Magnified images of a diffracted order at different focal positions indicate that astigmatism is predominant. The relationship of the sagittal, medial, and tangential surface to the observation hemisphere is also shown. 
This relationship is valid provided that the observation space is a hemisphere centered on the diffracting aperture and if the incident radiation is a unit amplitude spherical wave whose center of curvature lies on the intersection of the observation hemisphere with the z-axis. Furthermore, if $r$ is large compared to the size of the diffracting aperture, the Fourier transform relationship is accurate, not merely over a small region about the $z$-axis, but instead over the entire hemisphere.

Now consider the situation where the incident radiation strikes the diffracting aperture at an angle $\theta_{0}$ as illustrated in Fig. 2.7. This is equivalent to introducing a linear phase variation across the aperture. By applying the shift theorem (Bracewel1, 1965) of Fourier transform theory to Eq. (29) we find that the complex amplitude distri-. bution on the hemisphere is a function of $\left(\beta-\beta_{\beta}\right)$,

$$
U\left(\alpha, \beta-\beta_{O} ; \hat{x}\right)=\gamma \frac{e^{i 2 \pi \hat{r}}}{i \hat{x}}\left\{\left(T(\hat{x}, \hat{y} ; 0) \exp \left[i 2 \pi \beta_{O} \hat{y}\right]\right\},\right.
$$

where $B$ is the direction cosine of the position vector of the observation point, and $\beta_{O}$ is the direction cosine of the position vector of the undiffracted beam. Note that these direction cosines are obtained by merely projecting the respective points on the hemisphere back on to the plane of the aperture and normalizing to a unit radius. The complex amplitude distribution at an arbitrary point on the hemisphere can now be said to be a function of the distance of the observation point from the undiffracted beam in direction cosine space. 


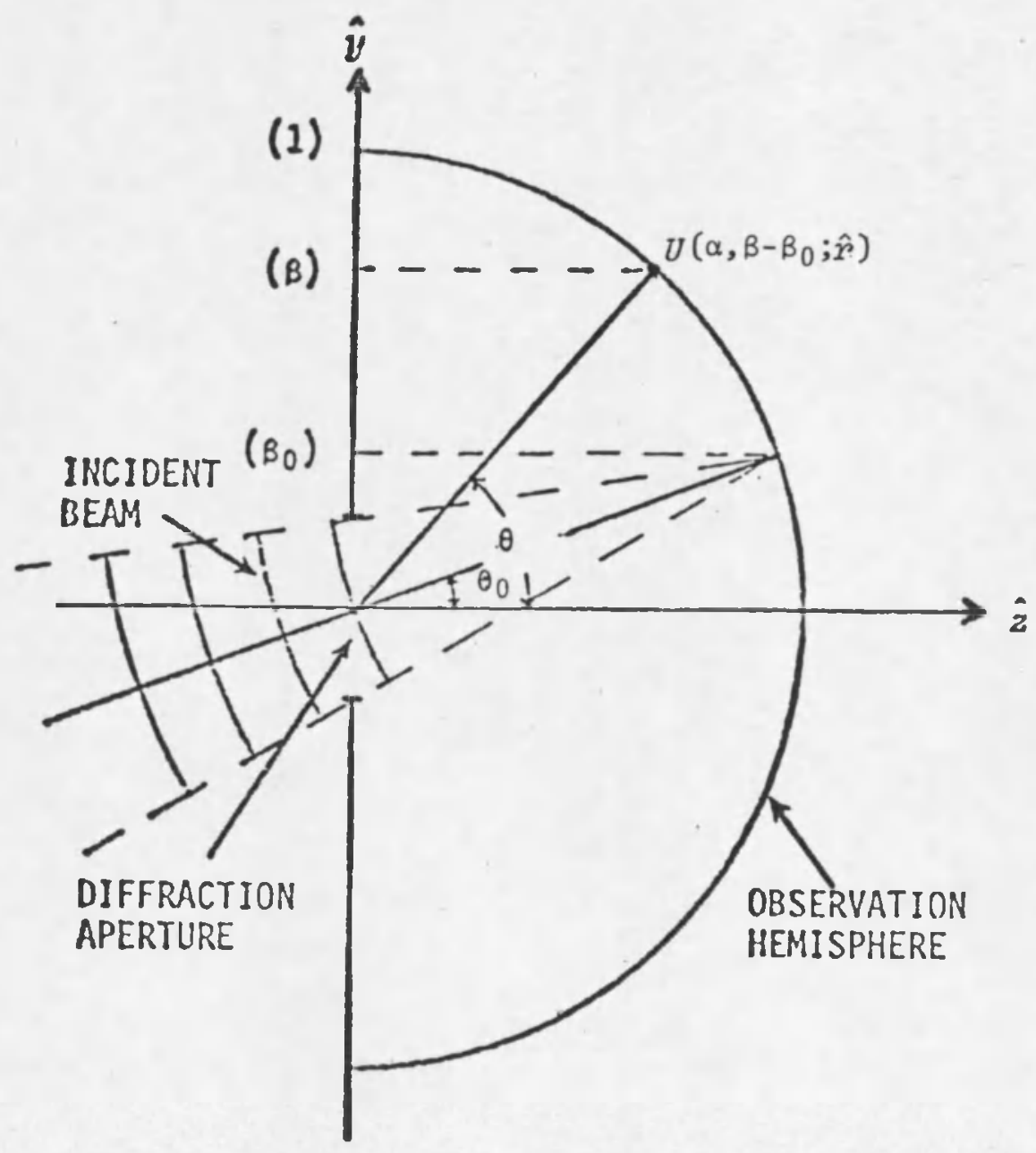

Fig. 2.7. Geometrical Configuration when the Incident Beam Strikes the Diffraction Aperture at an Arbitrary Angle. 
As a specific example, suppose we have incident light striking a diffraction grating at an angle $\theta_{O}$. The diffracted orders will strike the observation hemisphere in a cross section which is not a great circle but instead a latitude slice as shown in Fig. 2.8. Thus for large angles of incidence the various orders appear to 1 ie in a straight line only if they are projected down onto the $\alpha-\beta$ plane in direction cosine space. It is therefore clear that varying the angle of incidence merely shifts the diffracted wave field in direction cosine space without changing its functional form. This has been verified experimentally by mounting a diffraction grating at the center of a transparent hemisphere, placing graph paper on the plane of the diffraction grating ( $\alpha-\beta$ plane), and scribing appropriate latitude lines on the hemisphere upon which the diffracted orders fall when illuminated with a sma11 laser beam.

\section{Summary}

We have developed a very useful treatment of near-field scalar diffraction theory that yields much more insight than the conventional Rayleigh-Sommerfeld theory.

By describing the diffraction process in terms of the direction cosines of the propagating light we have obtained the extremely powerful result that the diffracted wave field on an observation hemisphere is given directly by the Fourier transform of the aperture function, This allows us to apply the well-known techniques of linear systems theory that have proven so useful in the area of image formation. 


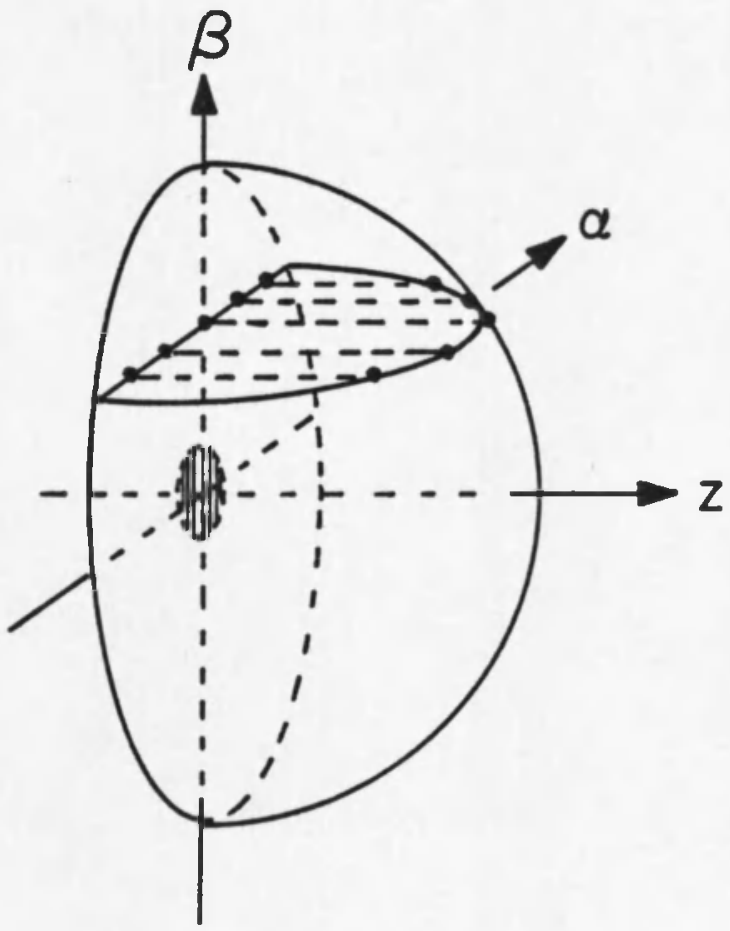

Fig. 2.8. Illustration of the Position of the Diffracted Orders in Real Space and Direction Cosine Space. 
Furthermore, we have shown that any departures of the actual diffracted wave field from those predicted by the Fourier transform. relationship take the form of conventional aberrations whose behavior is well understood in terms of the dimensions of the diffraction aperture, the radius of the observation hemisphere, and the appropriate field parameters. 
CHAPTER 3

\section{SURFACE SCATTER THEORY}

In the following treatment the scattering of light from optical elements is considered to be solely a surface phenomenon. It is recognized that bulk scattering mechanisms, such as photon-phonon interactions (Bloembergen and Shen, 1965) and scattering from free electrons (Vachaspati, 1964), can exist if the substrate material is not perfectly conducting. The excitation of surface plasmons has also been suggested by several investigators as contributing to short wavelength scattering from polished metal surfaces (Beaglehole, 1970; Beaglehole and Hunderi, 1970; Crowell and Ritchie, 1970; Elson and Ritchie, 1971; and Daudé, Savary, and Robin, 1972). However, the above effects are believed to be small for most visible and infrared radiation scattered from metal surfaces.

\section{Surface Scatter Phenomena as a Diffraction Process}

In Chapter 2 it was shown that, under the proper circumstances, the diffracted wave field on a hemisphere is given directly by the Fourier transform of the complex amplitude transmittance of the diffracting aperture. Usually a diffracting aperture consists of a "hole" in some opaque surface. This is a binary amplitude diffracting aperture. Clearly, a continuous amplitude diffracting aperture (a piece of photographic film, for example) can also exist. A more general situation is the complex diffracting aperture which exhibits both amplitude and 
phase variations. It is these phase perturbations that lead to scattered radiation. Surface scatter phenomena can thus be described as a diffraction process in which the pupil function has random phase variations in addition to any existing amplitude variations. The diffraction theory of the previous chapter can therefore be applied directiy to the problem of predicting the complex amplitude distribution on an observation hemisphere of radius $r$ resulting from an incident beam of light being reflected from a rough surface.

The System Pupil Function

A simple treatment of surface scatter theory can be formulated by considering the effect of the scattering surface to be a spacedependent modifier, or random component, of the effective pupil function of the system. The disturbance emerging from the scattering surface is then given by

$$
U_{O}(\hat{x}, \hat{y} ; 0)=a_{O} p(\hat{x}, \hat{y} ; 0)=a_{O} p_{L}(\hat{x}, \hat{y}: 0) P_{R}(\hat{x}, \hat{y} ; 0) .
$$

Here the pupil function of the system producing the incident beam is given by

$$
p_{L}(\hat{x}, \hat{y} ; 0)=a_{L}(\hat{x}, \hat{y} ; 0) e^{i 2 \pi \hat{W}_{L}(\hat{x}, \hat{y} ; 0)},
$$

where $\alpha_{L}$ describes the amplitude variations across the exit pupil of the system, and $W_{L}$ describes any phase variations or aberrations in the wavefront of the incident beam.

The random component of the pupil function due to the scattering surface similarly has an amplitude and phase component 


$$
p_{R}(\hat{x}, \hat{y} ; 0)=\sqrt{R(\hat{x}, \hat{y} ; 0)} \mathrm{e}^{i 4 \pi \hat{W}_{R}(\hat{x}, \hat{y} ; 0)} .
$$

Here $R$ is the reflectance of the scattering surface and $\hat{W}_{R}$ is the surface height. Note that the phase variations on the reflected wavefront are twice as large as the actual variations on the reflecting surface.

Figure 3.1 illustrates the surface height variations, $\hat{W}_{R}$, as a function of distance along the surface. This surface profile has associated with it an autocovariance function and a surface height distribution function as indicated.

Surface

height Surface

distribution
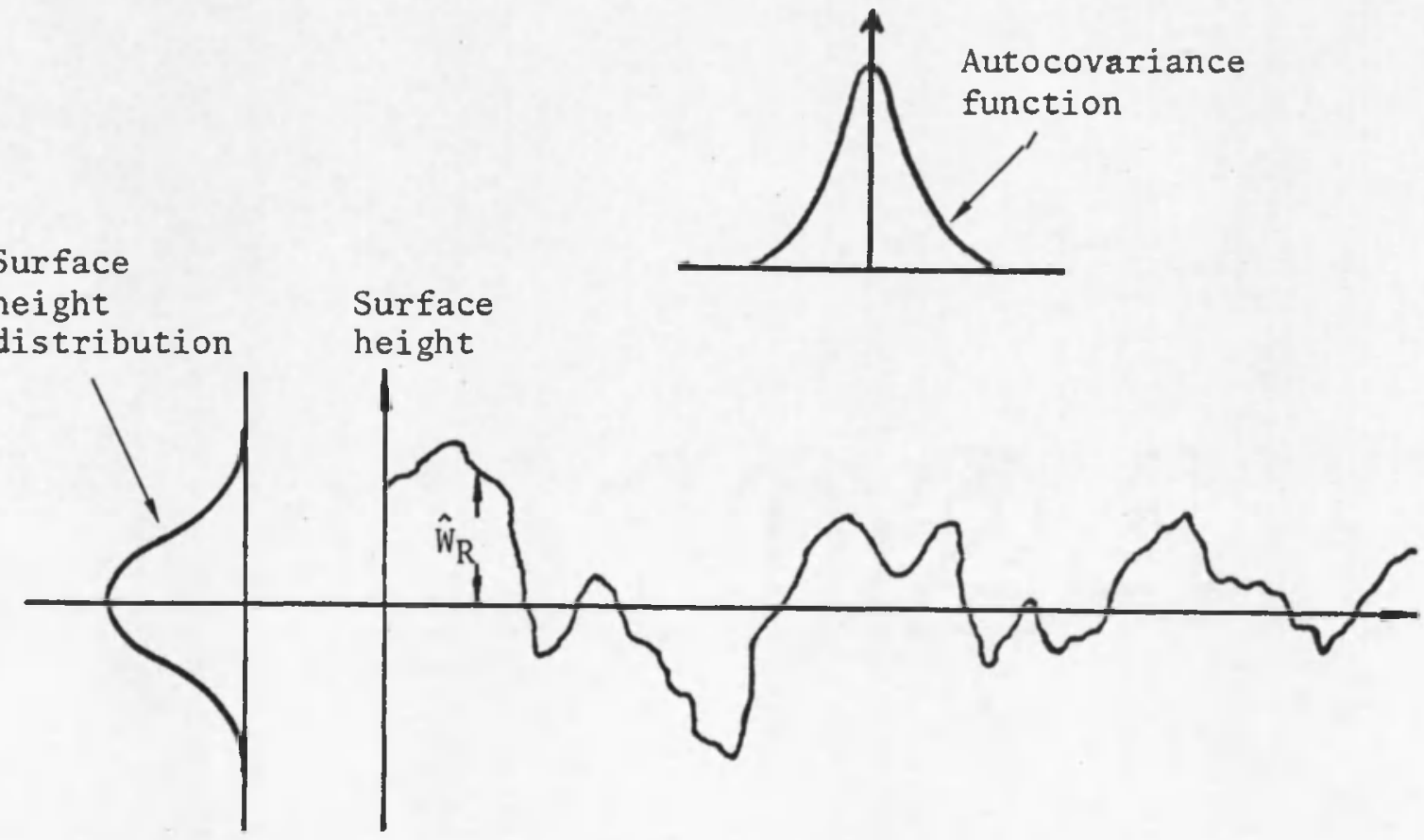

Fig. 3.1. Illustration of Surface Height Variations and Associated Statistical Parameters. 
Intensity Distribution of a Scattering System

The results of the previous chapter, [Eq. (29)], indicate that the complex amplitude distribution on the observation hemisphere is given directly by the Fourier transform of $U_{0}(\hat{x}, \hat{y} ; 0)$

$$
U(\alpha, \beta ; \hat{r})=\gamma \frac{e^{i 2 \pi \hat{r}}}{i \hat{r}} \mathscr{F}\left\{U_{O}(\hat{x}, \hat{y} ; 0)\right\} .
$$

The total reflected flux $\Phi$ is obtained by applying Rayleigh's theorem from Fourier transform theory

$$
\Phi=\int_{-\infty}^{\infty} \int_{r^{2}} \frac{\hat{r}^{2}}{r^{2}}|U(\alpha, \beta ; \hat{x})|^{2} d \alpha d \beta=a_{0}^{2} \iint_{-\infty}^{\infty}\left|p\left(\hat{x}^{\prime}, \hat{y}^{\prime} ; 0\right)\right|^{2} d \hat{x}^{\prime} d \hat{y}^{\prime}
$$

Noting that $d w=d a d \beta / \gamma$, the radiant intensity of the scattering system can be written as

$$
I(\alpha, \beta)=\frac{d \Phi}{d \omega}=\frac{\hat{r}^{2}}{\gamma}|U(\alpha, \beta ; \hat{r})|^{2}=a_{0}{ }^{2} \gamma|S\{p(\hat{x}, \hat{y} ; 0)\}|^{2} .
$$

Utilizing the autocorrelation theorem of Fourier transform theory, this is equivalent to

$$
I(\alpha, \beta)=a_{0}^{2} \gamma F\left\{\iint_{-\infty}^{\infty} p\left(\hat{x}^{\prime}, \hat{y}^{\prime} ; 0\right) p^{*}\left(\hat{x}^{\prime}-\hat{x}, \hat{y}^{\prime}-\hat{y} ; 0\right) d \hat{x}^{\prime} d \hat{y}^{\prime}\right\}
$$

For the special case of a Lambertian surface, the autocorrelation function approaches a Dirac $\delta$-function; hence, its Fourier transform is constant and we obtain

$$
I_{L}(\alpha, \beta)=\gamma K, \quad K=\text { constant }
$$

which is consistent with Lambert's cosine law. 
The System Spread Function

Following the standard procedure used in image evaluation, the effective transfer function of the scattering system is defined as the normalized autocorrelation of the pupil function

$$
\mathscr{H}(\hat{x}, \hat{y} ; 0)=\frac{\iint_{-\infty}^{\infty} p\left(\hat{x}^{\prime}, \hat{y}^{\prime} ; 0\right) p^{*}\left(\hat{x}^{\prime}-\hat{x}, \hat{y}^{\prime}-\hat{y} ; 0\right) d \hat{x}^{\prime} d \hat{y}^{\prime}}{\iint_{-\infty}^{\infty}\left|p\left(\hat{x}^{\prime}, \hat{y}^{\prime} ; 0\right)\right|^{2} d \hat{x}^{\prime} d \hat{y}^{\prime}} .
$$

The effective spread function of the scattering system is now defined in the usual way as the Fourier transform of the transfer function

$$
\boldsymbol{\delta}(\alpha, \beta)=\mathscr{F}\{\mathscr{H}(\hat{x}, \hat{y} ; 0)\}=\frac{\mathscr{F}\left\{\iint_{-\infty}^{\infty} p\left(\hat{x}^{\prime}, \hat{y}^{\prime} ; 0\right) p^{*}\left(\hat{x}^{\prime}-\hat{x}, \hat{y}^{\prime}-\hat{y} ; 0\right) d \hat{x}^{\prime} d \hat{y}^{\prime}\right\}}{\iiint_{-\infty}^{\infty}\left|p\left(\hat{x}^{\prime}, \hat{y}^{\prime} ; 0\right)\right|^{2} d \hat{x}^{\prime} d \hat{y}^{\prime} \quad \text { (40) }}
$$

Direct substitution from Eq. (35) and Eq. (38) results in the following expression for the effective spread function in terms of the radiant intensity of the scattering system

$$
\delta(\alpha, \beta)=\frac{1}{\gamma \Phi} I(\alpha, \beta)=\frac{I(\alpha, \beta)}{\Phi\left(1-\alpha^{2}-\beta^{2}\right)^{\frac{1}{2}}} .
$$

\section{Scattering from Optical Surfaces}

Let the height variations $\hat{W}_{R}$ of a given illuminated area on a scattering surface be a two-dimensional sample function $\hat{W}_{R i}(\hat{x}, \hat{y} ; 0)$. A 
random process, $\hat{W}_{R}(\hat{x}, \hat{y} ; 0)$, is made up of an ensemble of such functions as shown in Fig. 3.2. For fixed spatial coordinates, $\hat{W}_{R}\left(\hat{x}_{I}, \hat{y}_{1} ; 0\right)$ is a random variable. And, for a specific sample function with fixed spatial coordinates, $\hat{W}_{R i}\left(\hat{x}_{1}, \hat{y}_{1} ; 0\right)$ is a single number.

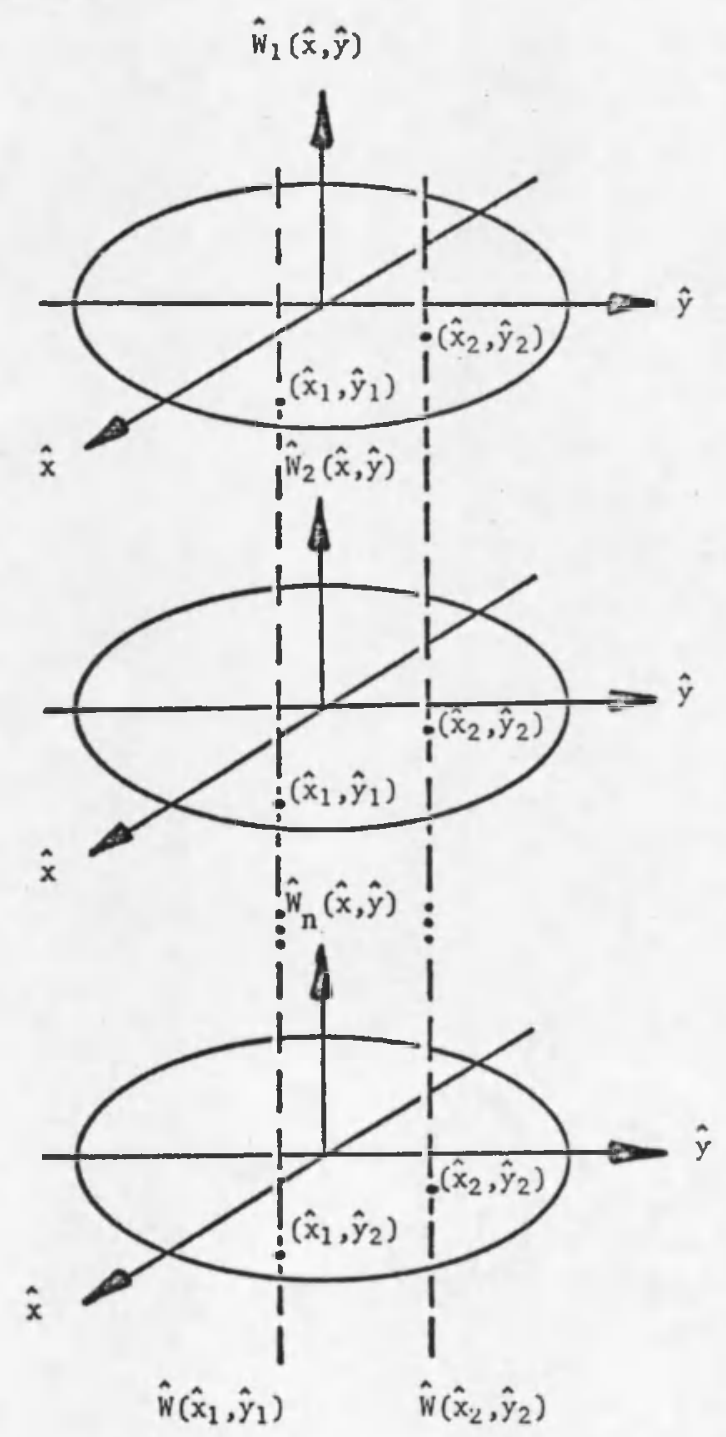

Fig. 3.2. An Ensemble of Two-dimensional Sample Functions $\hat{W}_{i}(\hat{x}, \hat{y})$ Representing Surface Height Variations Constitutes a Random Process $\hat{W}(\hat{x}, \hat{y})$.

Two random variables, $\hat{W}\left(\hat{x}_{1}, \hat{y}_{1}\right)$ and $\hat{W}\left(\hat{x}_{2}, \hat{y}_{2}\right)$ with fixed spatial coordinates are also shown. 
The Statistical Properties of an Optical Surface

We are primarily interested in the scattering behavior of optical surfaces. The following assumptions are made concerning the statistical properties of an optical surface prepared by conventional fabrication techniques on ordinary optical materials:

1. The reflectance $R$ is constant over the entire surface. This assumption is not essential but it is reasonable and furthermore it has been shown by Shack (1967) that phase fluctuations will dominate over amplitude fluctuations in their combined effect on the spread function.

2. $\hat{W}_{R}(\hat{x}, \hat{y} ; 0)$ is a single-valued Gaussian random process.

3. $\hat{W}_{R}(\hat{x}, \hat{y} ; 0)$ is at least locally stationary in the statistical sense (i.e., surface is homogeneous and isotropic).

4. The random variables $\hat{W}_{R}\left(\hat{x}_{1}, \hat{y}_{1} ; 0\right)$ and $\hat{W}_{R}\left(\hat{x}_{2}, \hat{y}_{2} ; 0\right)$, produced by any two fixed pairs of spatial coordinates, are jointly normal.

5. $\hat{w}_{R}(\hat{x}, \hat{y} ; 0)$ is weakly ergodic (i.e., the mean and autocorrelation function determined by space averages using a single sample function $\hat{W}_{R i}(\hat{x}, \hat{y} ; 0)$ are the same as those determined by ensemble averages). 
The Transfer Function of a Scattering Surface

Substituting Eq. (31) into Eq. (39) we obtain

$$
\mathscr{H}(\hat{x}, \hat{y} ; 0)=\frac{\iint_{L 1}^{\infty} a_{L 2} e^{i 2 \pi\left[\hat{W}_{L 1}-\hat{W}_{L 2}\right]} e^{i 4 \pi\left[\hat{W}_{R 1}-\hat{W}_{R 2}\right]} d \hat{x}^{\prime} d \hat{y}^{\prime}}{\iint_{-\infty}^{\infty}\left|a_{L 1}\right|^{2} d \hat{x}^{\prime} d \hat{y}^{\prime}}
$$

where

$$
\begin{aligned}
& a_{L 1}=a_{L}\left(\hat{x}^{\prime}, \hat{y}^{\prime} ; 0\right) \\
& a_{L 2}=a_{L}\left(\hat{x}^{\prime}-\hat{x}, \hat{y}^{\prime}-\hat{y} ; 0\right) \\
& \hat{W}_{L 1}=\hat{W}_{L}\left(\hat{x}^{\prime}, \hat{y}^{\prime} ; 0\right) \\
& \hat{W}_{L 2}=\hat{W}_{L}\left(\hat{x}^{\prime}-\hat{x}, \hat{y}^{\prime}-\hat{y} ; 0\right) \\
& \hat{W}_{R I}=\hat{W}_{R}\left(\hat{x}^{\prime}, \hat{y}^{\prime} ; 0\right) \\
& \hat{W}_{R 2}=\hat{W}_{R}\left(\hat{x}^{\prime}-\hat{x}, \hat{y}^{\prime}-\hat{y} ; 0\right) .
\end{aligned}
$$

The above expression for the transfer function contains the random variables $W_{R 1}$ and $W_{R 2}$; therefore, taking the expected value we have

$$
E\{\mathscr{H}(x, y ; 0)\}=\frac{\iint_{L 1}^{\infty} a_{L 2} \mathrm{e}^{i 2 \pi\left[\hat{W}_{L I}-\hat{\hat{W}}_{L 2]}\right]} E\left\{\mathrm{e}^{i 4 \pi\left[\hat{W}_{R 1}-\hat{W}_{R 2}\right]}\right\} d \hat{x}^{\prime} d \hat{y}^{\prime}}{\iint_{-\infty}^{\infty}\left|a_{L}\right|^{2} d \hat{x}^{\prime} d \hat{y}^{\prime}}
$$


Since the random variables involved are assumed to be stationary, the expected value under the integral is independent of $\hat{x}^{\prime}$ and $\hat{y}^{\prime}$ and can be taken outside the integral

$$
E\{\mathscr{H}(\hat{x}, \hat{y} ; 0)\}=E\left\{e^{i 4 \pi\left[\hat{W}_{R 1}-\hat{W}_{R 2}\right]}\right\} \frac{\iint_{-\infty}^{\infty} a_{L 1} a_{L 2} e^{i 2 \pi\left[\hat{W}_{L 1}-\hat{W}_{L 2}\right]} d \hat{x}^{\prime} d \hat{y}^{\prime}}{\iint_{-\infty}^{\infty}\left|a_{L}\right|^{2} d \hat{x}^{\prime} d \hat{y}^{\prime}} .
$$

We now recognize the normalized autocorrelation function in the above equation to be the transfer function of the optical system producing the incident beam

$$
\mathscr{H}_{L}(\hat{x}, \hat{y} ; 0)=\frac{\iint_{-\infty}^{\infty} a_{L 1} a_{L 2} e^{i 2 \pi\left[\hat{W}_{L 1}-\hat{W}_{L 2}\right]} d \hat{x}^{\prime} d \hat{y}^{\prime}}{\iint_{-\infty}^{\infty}\left|a_{L}\right|^{2} d \hat{x}^{\prime} d \hat{y}^{\prime}} .
$$

The average quantity in Eq. (45) is therefore the equivalent transfer function of the scattering surface

$$
\mathscr{X}_{R}(\hat{x}, \hat{y} ; 0)=E\left\{e^{i 4 \pi\left[\hat{W}_{R 1}-\hat{W}_{R 2}\right]}\right\} .
$$

But this is merely the joint characteristic function (Papoulis, 1965 , p. 225) of the two random variables $\hat{W}_{R 2}$ and $\hat{W}_{R 2}$. Since $\hat{W}_{R 1}$ and $\hat{W}_{R 2}$ are jointly normal randon variables, it can be shown 
that (see Papoulis, 1965, p. 226)

$$
E\left\{\mathrm{e}^{i 4 \pi\left[\hat{W}_{R \perp-}-\hat{W}_{R 2}\right]}\right\}=\mathrm{e}^{i 4 \pi\left(\eta_{1}-\eta_{2}\right)} \mathrm{e}^{-8 \pi^{2}\left(\sigma_{1}{ }^{2}-2 C_{12}+\sigma_{2}{ }^{2}\right)}
$$

where

$$
C_{12} \equiv E\left\{\left(\hat{W}_{R 1}-n_{1}\right)\left(\hat{W}_{R 2}-n_{2}\right)\right\}
$$

is the covariance function of the random variables $\hat{W}_{R 1}$ and $\hat{W}_{R 2}$. But $\hat{W}_{R I}$ and $\hat{W}_{R 2}$ are identical functions merely displaced from one another; hence

$$
\sigma_{1}=\sigma_{2}=\sigma_{\hat{W}^{\prime}} \quad \eta_{1}=n_{2}
$$

and

$$
C_{12} \equiv C_{\hat{W}}(\hat{x}, \hat{y}) \equiv \text { autocovariance of } \hat{W}_{R} \text {. }
$$

The equivalent transfer function of the scattering surface is thus given by

$$
\mathscr{H}_{R}(\hat{x}, \hat{y} ; 0)=e^{-(4 \pi \sigma \hat{W})^{2}}\left[1-\frac{C_{\hat{W}}(\hat{x}, \hat{y})}{\sigma_{\hat{W}}{ }^{2}}\right]
$$

where $\sigma_{\hat{W}}^{2}$ is the variance of the surface height distribution function and $C_{\hat{W}}(\hat{x}, \hat{y})$ is the two-dimensional autocovariance function of the surface.

Considerable insight into the scattering process can now be obtained by considering the nature of this transfer function. The autocovariance function approaches the value $\sigma \hat{W}^{2}$ as the displacement 
approaches zero. The equivalent transfer function thus approaches unity as expected. As the displacement approaches infinity the autocovariance function approaches zero and the equivalent transfer function approaches a plateau of height $\exp \left[-(2 \pi \sigma \hat{W})^{2}\right]$.

The equivalent transfer function of the scattering surface can thus be regarded as the sum of a constant component and a bell-shaped component as shown in Fig. 3.3(a). Equation (52) can therefore be rewritten as

$$
\mathscr{X}_{R}(\hat{x}, \hat{y} ; 0)=A+B Q(\hat{x}, \hat{y} ; 0) \text {, }
$$

where

$$
\begin{aligned}
A & =e^{-(4 \pi \sigma \hat{W})^{2}} \\
B & =1-e^{-(4 \pi \sigma \hat{W})^{2}} \\
Q(\hat{x}, \hat{y} ; 0) & =\frac{e^{(4 \pi \sigma \hat{W})^{2} \frac{C_{\hat{W}}(\hat{x}, \hat{y})}{\sigma_{\hat{W}}^{2}}}-1}{e^{(4 \pi \sigma \hat{W})^{2}}-1} .
\end{aligned}
$$

The Spread Function of a Scattering Surface

The significance of this interpretation of the equivalent transfer function of the scattering surface is dramatically shown by the inferred properties of the corresponding spread function. Since the 


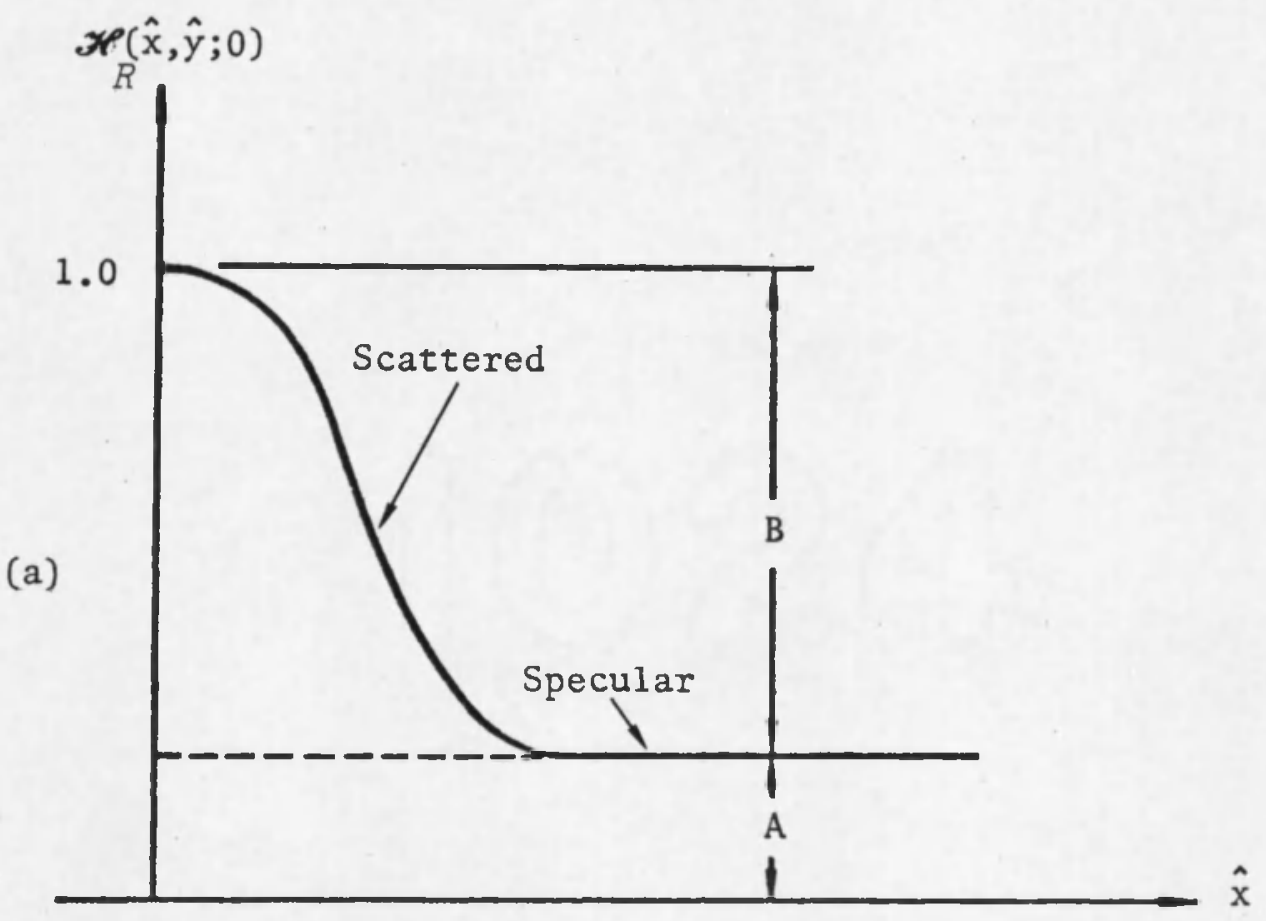

(b)

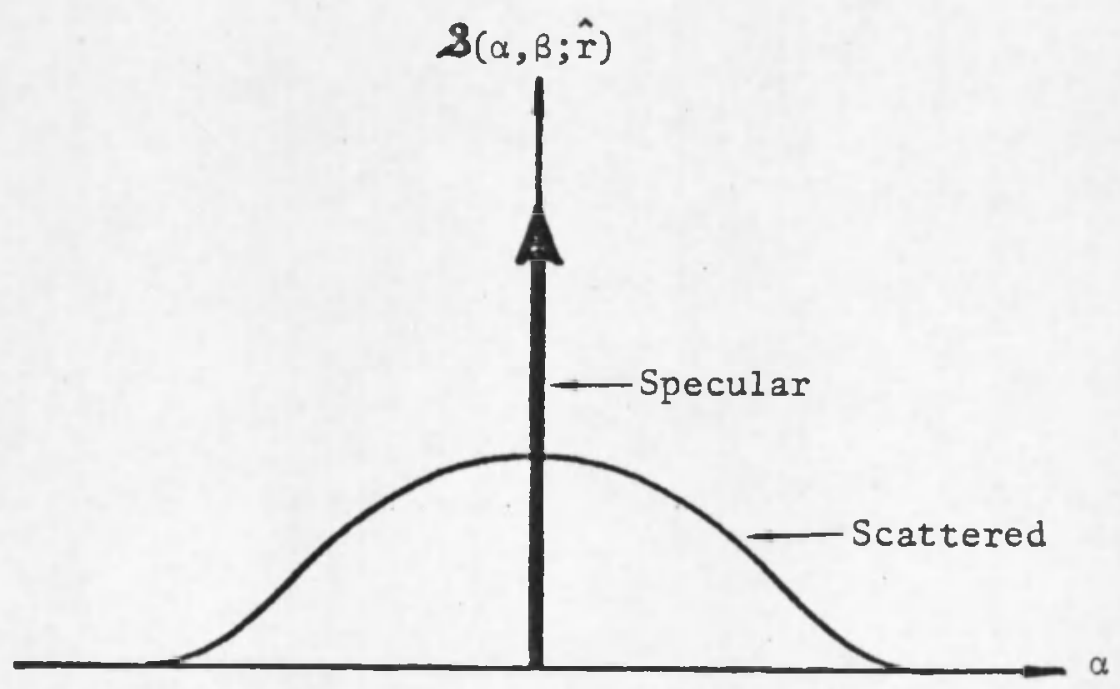

Fig. 3.3. Illustration of the Effective Transfer Function and Spread Function of a Scattering Surface.

(a) Transfer function.

(b) Spread function. 
transfer function is the sum of two separate components, the equivalent spread function of the scattering surface is the sum of the inverse Fourier transforms of the two component functions,

$$
\mathscr{B}\left(\alpha, \beta ; \hat{x^{\prime}}\right)=\mathscr{F}^{-1}\left\{\mathscr{H}_{R}(\hat{x}, \hat{y} ; 0)\right\}=A \delta(\alpha, \beta ; \hat{r})+S(\alpha, \beta ; \hat{r})
$$

where

$$
S(\alpha, \beta ; \hat{r})=B \bar{F}-1\{Q(\hat{x}, \hat{y} ; 0)\} .
$$

The constant component transforms into a delta function, and the be11shaped component transforms into a bell-shaped scattering function as shown in Fig. 3.3(b). Hence the scattering surface reflects an incident beam of light as a specularly-reflected beam of diminished intensity surrounded by a halo of scattered light. Furthermore, the relative power distribution between the specular component and the scattered component of the effective spread function are given by the quantities $A$ and $B$ respectively.

Note that as more and more light is scattered, energy is transferred from the specular component of the spread function into the scattered component of the spread function. For a perfectly Lambertian reflector the specular component disappears completely from the spread function.

A Shift-invariant Scattering Function

In general, the scattered light distribution on an observation hemisphere will change with the angle of the incident light just as the point spread function of an optical imaging system will, in 
genera1, vary with the field position of the point source. However, the analysis of imaging systems is greatly simplified by assuming an isoplanatic system in which the point spread function does not change with field position (and this is a reasonable assumption for many practical imaging systems). Similarly, the analysis of light scattering systems will be greatly simplified if they can be shown to be shift-invariant (i.e., if the shape of the scattering function does not change with the angle of incidence).

From the discussion in the previous section it is clear that the scattered light distribution on an observation hemisphere will appear to consist of the sum of two components, a core which is the delta function convolved with the spread function of the optical system producing the incident beam, and a scattering function which is the bell-shaped halo convolved with the spread function of the optical system.

In Fig. 3.4 we have merely replaced the diffracting aperture of Fig. 2.7, page 31, with a scattering surface and the geometry of the measurements has been folded about the reflecting plane. Hence, we have the incident beam striking the scattered surface at some angle of incidence, a specularly-reflected beam striking the observation hemisphere, and the scattered light distribution being sampled at an arbitrary point with direction cosine coordinates $\alpha$ and $\beta$. The scattered light distribution on the hemisphere wi.1, in general, change shape drastically with angle of incidence--becoming quite skewed and asymmetrical at large angles of incidence. However, our theory 


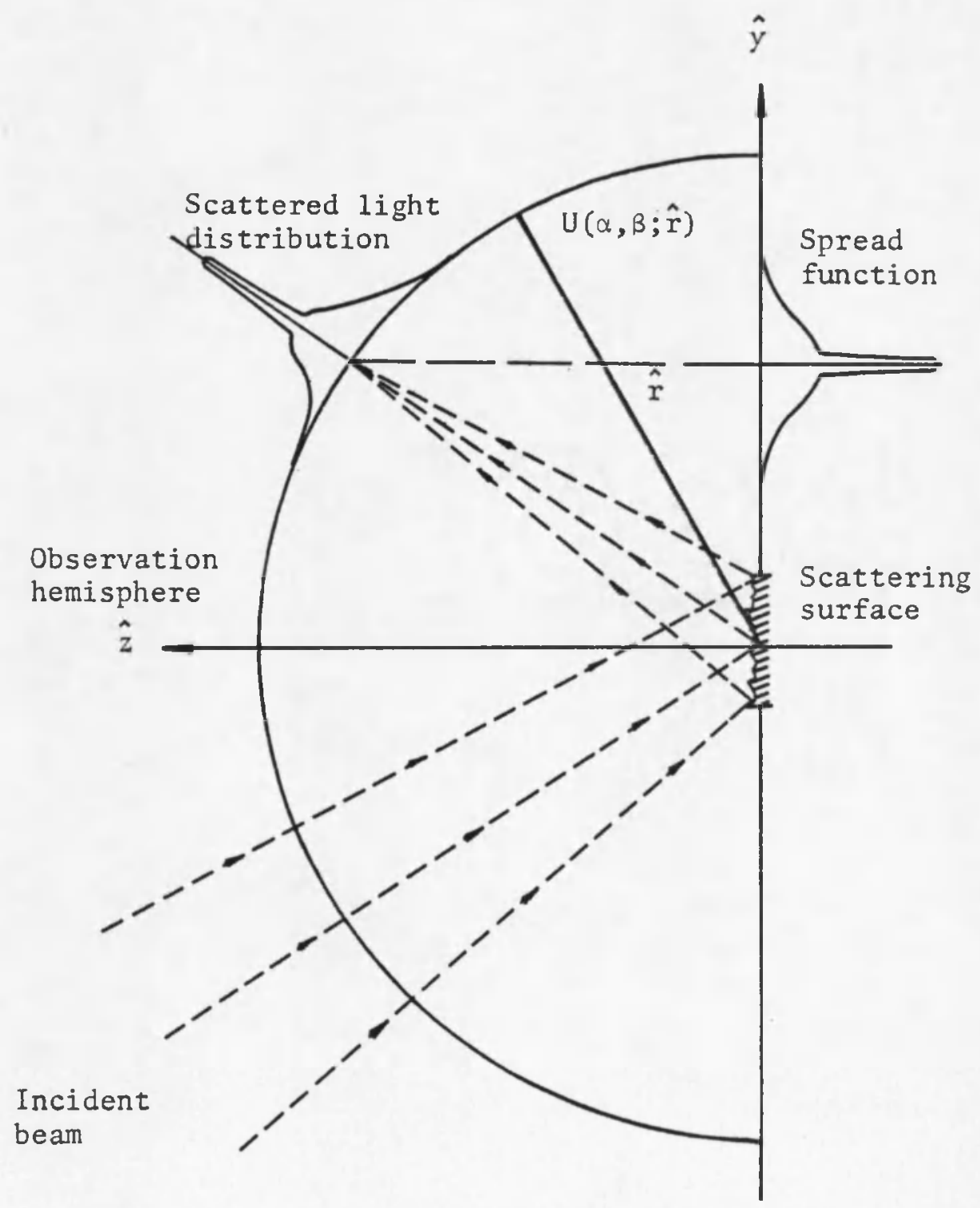

Fig. 3.4. Geometrical Relationship Between Incident Beam, Scattering Surface, the Measured Scattered Light Distribution, and the Resulting Spread Function. 
indicates that the data collected on the hemisphere should be plotted as a function of the direction cosines of the position vector of the observation point. For certain surfaces with well-behaved statistics, this new scattering function will not change shape but will merely be shifted in direction cosine space with changes in angle of incidence. The four-dimensional Bidirectional Reflectance Distribution Function (BRDF), which is the basic quantity that completely characterizes the scattering properties of a surface (see Appendix B), will therefore degenerate into a single two-dimensional spread function. This is a rather significant development which has profound implications regarding the quantity of data required to completely characterize a scattering surface. However, it remains to be experimentally verified that scattering surfaces of practical interest obey these predictions.

The Inverse Scattering Problem

The problem of determining surface characteristics from scat-tered light measurements is frequently referred to as the inverse scattering problem. A general treatment of electromagnetic inverse scattering has been discussed by Bojarski (1971). Several attempts have been made (Daniels, 1961; Fung and Moore, 1964; and Barrick, 1965) to determine properties of the lunar surface by applying inverse scattering techniques to radar returns from the moon. Bennett and Bennett (1967) were able to obtain the rms roughness and autocovariance length of the surface structure of mirrors by assuming a Gaussian shape for both the surface height distribution function and the surface autocovariance function. More recently Scheele (1973) met with little 
success in attempting to ascertain under what conditions the exact autocovariance function can be obtained from scattered light data.

The treatment presented in this chapter describes surface scatter phenomena as a linear, shift-invariant process which is completely characterized by the effective transfer function of the scattering surface. Furthermore, for a large class of well-behaved surfaces this transfer function is described only in terms of the rms surface roughness and the surface autocovariance function and hence provides an elegant solution to the inverse scattering problem.

The surface autocovariance function is thus obtained from scattered light data by rewriting Eq. (52) as

$$
C_{W}(x, y)=\left(\frac{\lambda}{4 \pi}\right)^{2} \ln \left(\mathscr{H}_{R}\left(\frac{x}{\lambda}, \frac{y}{\lambda}\right)\right)+\sigma_{W}^{2}
$$

where

$$
\mathscr{H}_{R}\left(\frac{x}{\lambda}, \frac{y}{\lambda}\right)=\{\&(\alpha, \beta)\} \begin{aligned}
& \left.\right|_{\hat{x}=x / \lambda} \\
& \hat{y}=y / \lambda .
\end{aligned} .
$$

The rms surface roughness is given by

$$
\sigma_{W}=\frac{\lambda}{4 \pi} \sqrt{\ln \frac{1}{1-B}}
$$

where $B$ (the total integrated scatter expressed as a fraction of the total reflected light) is obtained by applying numerical integration techniques upon the measured data describing the scattered light distribution. Note that although we are limited by a scalar theory, we 
have made no explicit approximations regarding the size of the surface variations. If we make a smooth surface approximation $\left(\sigma_{W}<<\lambda\right)$, then the total integrated scatter is small $(B<<1)$ and we obtain the usual expression for the rms surface roughness

$$
\sigma_{W}=\frac{\lambda}{4 \pi} \sqrt{B}
$$

Wavelength Dependence of the Scattering Function

A successful theoretical model of surface scatter phenomena must provide a method of determining the wavelength dependence of the scattered light characteristics. This would allow one to infer the scattering behavior of a surface for any desired wavelength from a limited amount of data obtained at a given wavelength. Since the scattering mechanism is a diffraction process, it is clear that light of a particular wavelength scattered in a given direction corresponds to surface structure of a given spatial frequency. This spatial frequency component of the surface structure will scatter light of some other wavelength into a different direction. An analytical expression describing the wavelength dependence of the scattering function must therefore involve a change in the scatter angle with wavelength as we11 as the expected change in scattered intensity. Thus, if we wish to determine how the relative intensity at a fixed scatter angle varies with wavelength, the surface characteristics corresponding to the appropriate spatial frequency components of the surface structure must be known for each wavelength of interest. 
The above discussion perhaps explains the failure of various experimental investigators to agree in their attempts to establish the wavelength scaling behavior from empirical observations of scattered light data. Shack and DeBel1 (1974) made scattered light measurements on mirror surfaces at two visible wavelengths. Their data indicated a $1 / \lambda^{2}$ wavelength scaling 1 aw. Leinert and Klüpelberg (1974) also made mirror scatter measurements at two wavelengths in the visible. They found a $1 / \lambda$ wavelength dependence. Perkin-Elmex (1975) mirror scatter measurements were made at a visible wavelength and at $\lambda=10 \mu \mathrm{m}$. This data showed a $1 / \lambda^{\frac{1}{2}}$ wavelength scaling behavior.

The transfer function characterization of scattered surfaces developed in the previous section offers a simple means of determining this wavelength dependence. Once the surface characteristics are known [whether from direct measurement or calculated from scattered light data by means of Eq: (58) and Eq. (60)], the same theory provides an equally simple method of predicting the scattering function at any desired wavelength.

Since this technique involves numerical computations on sampled data, an analytical expression for the wavelength scaling law is not required. However, in order to gain more insight into the nature of surface scatter phenomena, we will proceed to derive the wavelength scaling law for the special case of a normally incident beam upon a smooth surface $\left(\sigma_{\left.W^{<}<\lambda\right)}\right.$. Under this condition the surface transfer function expressed in terms of real pupil coordinates $x$ and $y$ is given by 


$$
H_{R}(x, y)=A+\dot{B Q}(x, y)
$$

where

$$
\begin{aligned}
& A=1-\left(\frac{4 \pi \sigma_{W}}{\lambda}\right)^{2} \\
& B=\left(\frac{4 \pi \sigma_{W}}{\lambda}\right)^{2}
\end{aligned}
$$

and

$$
Q(x, y)=\frac{C_{W}(x, y)}{\sigma_{W}^{2}}
$$

From Eq. (57), we see that the scattering function for a particular wavelength is given by

$$
S(\alpha, \beta ; \lambda)=\left(\frac{4 \pi}{\lambda}\right)^{2} \mathscr{F}^{-1}\left\{C_{W}(\lambda \hat{x}, \lambda \hat{y})\right\}
$$

Applying the similarity theorem of Fourier transform theory we obtain

$$
S(\alpha, \beta ; \lambda)=\left(\frac{4 \pi}{\lambda}\right)^{2} \frac{1}{\lambda^{2}} \mathscr{C}_{W}\left(\frac{\alpha}{\lambda}, \frac{\beta}{\lambda}\right)
$$

where

$$
\mathscr{C}_{W}(\alpha, \beta)=\mathscr{F}^{-1} C_{W}(\hat{x}, \hat{y})
$$

If we now scale the wavelength by a factor $a$, we obtain

$$
S(\alpha, \beta ; \alpha \lambda)=\left(\frac{4 \pi}{a \lambda}\right)^{2} \frac{1}{(\alpha \lambda)^{2}} \mathscr{C}_{W}\left(\frac{\alpha}{a \lambda}, \frac{\beta}{a \lambda}\right)=\frac{1}{a^{4}} S\left(\frac{\alpha}{\alpha}, \frac{\beta}{\alpha} ; \lambda\right) .
$$

Therefore, the appropriate wavelength scaling law for smooth surfaces is given by

$$
S(\alpha, \beta ; \alpha \lambda)=\frac{1}{a^{4}} S\left(\frac{\alpha}{a}, \frac{\beta}{a} ; \lambda\right) .
$$

Note that, in addition to the $1 / a^{4}$ change in magnitude, the width of the scattering function in direction cosine space is scaled by the factor $a$. 
CHAPTER 4

\section{SURFACE SCATTER MEASUREMENTS}

\section{Apparatus}

An instrument has been designed and built at the Optical Sciences Center for making scattered light measurements on a hemisphere as described in the previous chapter. A schematic diagram of this apparatus is shown in Fig. 4.1. The incident light passes through.a chopper so that synchronous detection with a PAR lock-in amplifier can be made. The mechanical apparatus shown in Fig. 4.2 is located in a smal1 photometric darkroom in which the experiment is conducted. A movable arm with folding mirrors can be positioned to direct the incident beam onto the sample at any desired angle. A lens positioned on this arm focuses the incident radiation onto the hemisphere mapped out by the detector; hence, the geometrical configuration is consistent with that illustrated in Fig. 3.4 of the previous chapter. Two separate driving mechanisms allow us to measure the scattered light distribution over the entire hemisphere bounded by the plane of the sample.

The Light Source

The light source employed is a Spectra-Physics Mode1 165 Argon Ion Laser. The laser is operated with a light-regulated, singlefrequency output which assures intensity regulation to within 


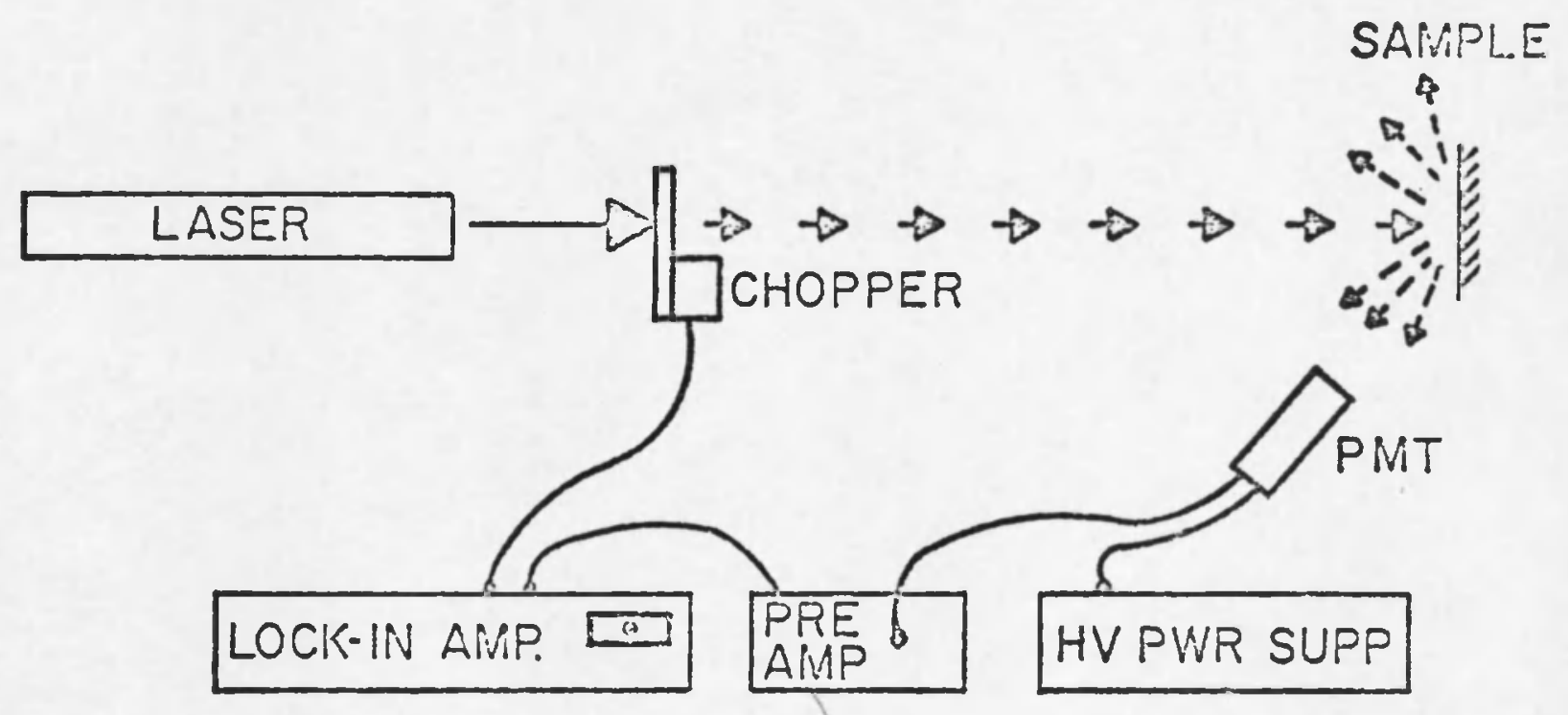

Fig. 4.1. Schematic Diagram of Scatter Measurement Apparatus. 


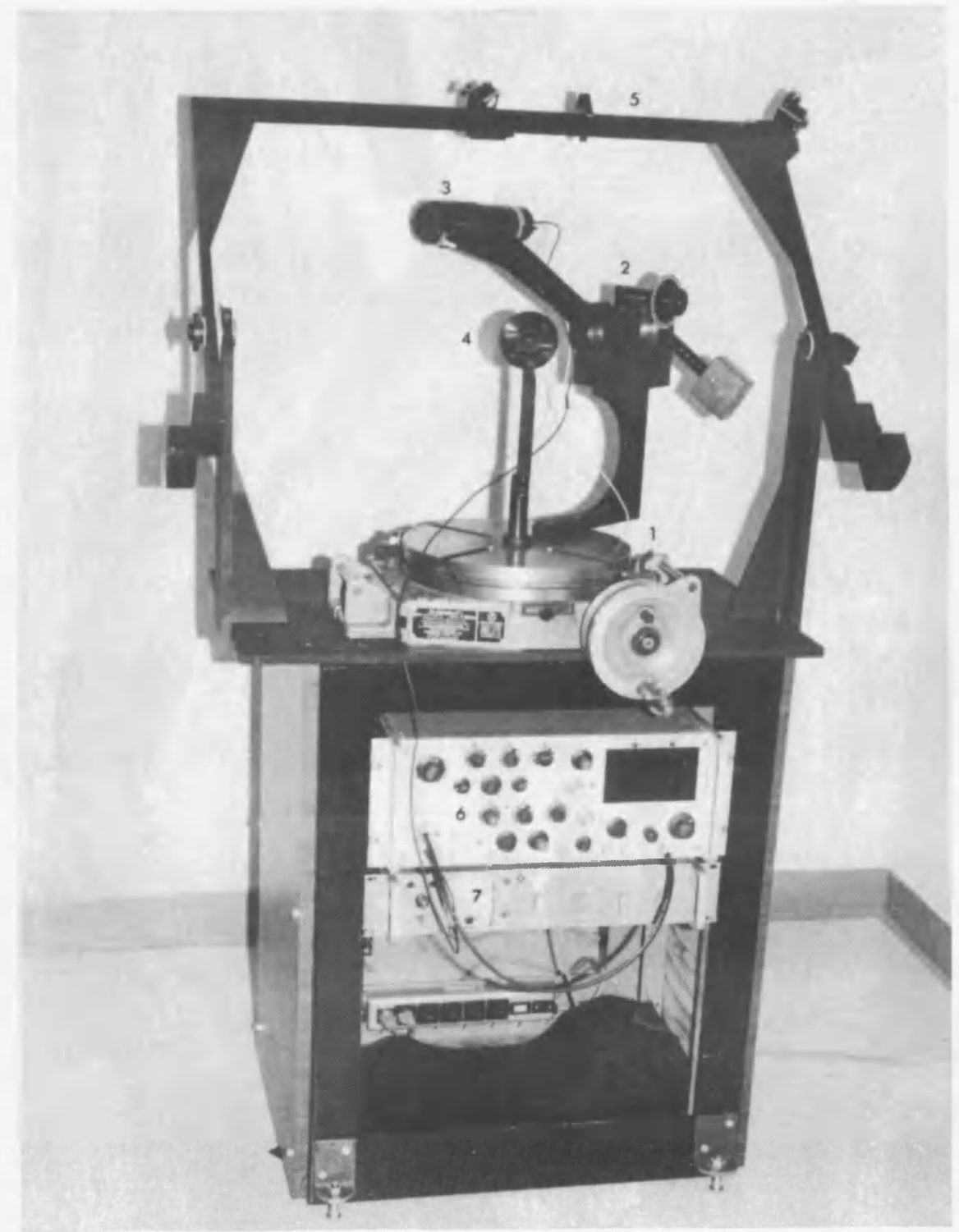

Fig. 4.2. Apparatus for Measuring Scattered Light Distribution from Optical Surfaces.

(1) Precision rotary table, (2) worm gear drive for arm supporting detector, (3) photomultiplier tube and fiberoptic probe, (4) sample holder, (5) movable arm with folding mirrors and lens for directing and focusing incident beam, (6) P.A.R. lock-in amplifier, (7) high voltage power supply for PMT. 
one percent. The measurements were made with approximately $20 \mathrm{mw}$ of power in the incident beam at wavelengths of $0.5145 \mu \mathrm{m}$ and $0.4579 \mu \mathrm{m}$. An He-Ne laser was also available for making measurements at a wavelength of $0.6328 \mu \mathrm{m}$.

The Detector Unit

The detector is a Philips one-inch, end-on photomultiplier tube (PMT) having an S-20 photocathode. Light reaches the photomultiplier by way of a rigid fiber-optic probe. Such a probe offers several distinct advantages in light sampling. In addition to allowing increased angular resolution throughout the sampling space, and enabling us to sample within 'one degree of the incident or specularly reflected beams, it provides the ability to control the field of view of the detector for the purpose of stray 1 ight rejection.

The original configuration consisted of a rigid fiber-optic bundle bent such that one end was pointed toward the illuminated spot on the sample. This end of the bundle thus acted as the collecting aperture for the detection system. The other end of the fiber-optic bundle protruded into the photomultiplier tube housing followed by a series of baffles to limit the field of view of the detector as shown in Fig. $4.3(\mathrm{a})$. This resulted in a detector response which had a Gaussian dependence upon field angle.

However, by introducing a small collecting lens and a field stop in front of the fiber-optic bundle as shown in Fig. 4.3(b), the baffles can be eliminated and a well-defined field of view of any desired size can be obtained by properly choosing the size of the 
(a)
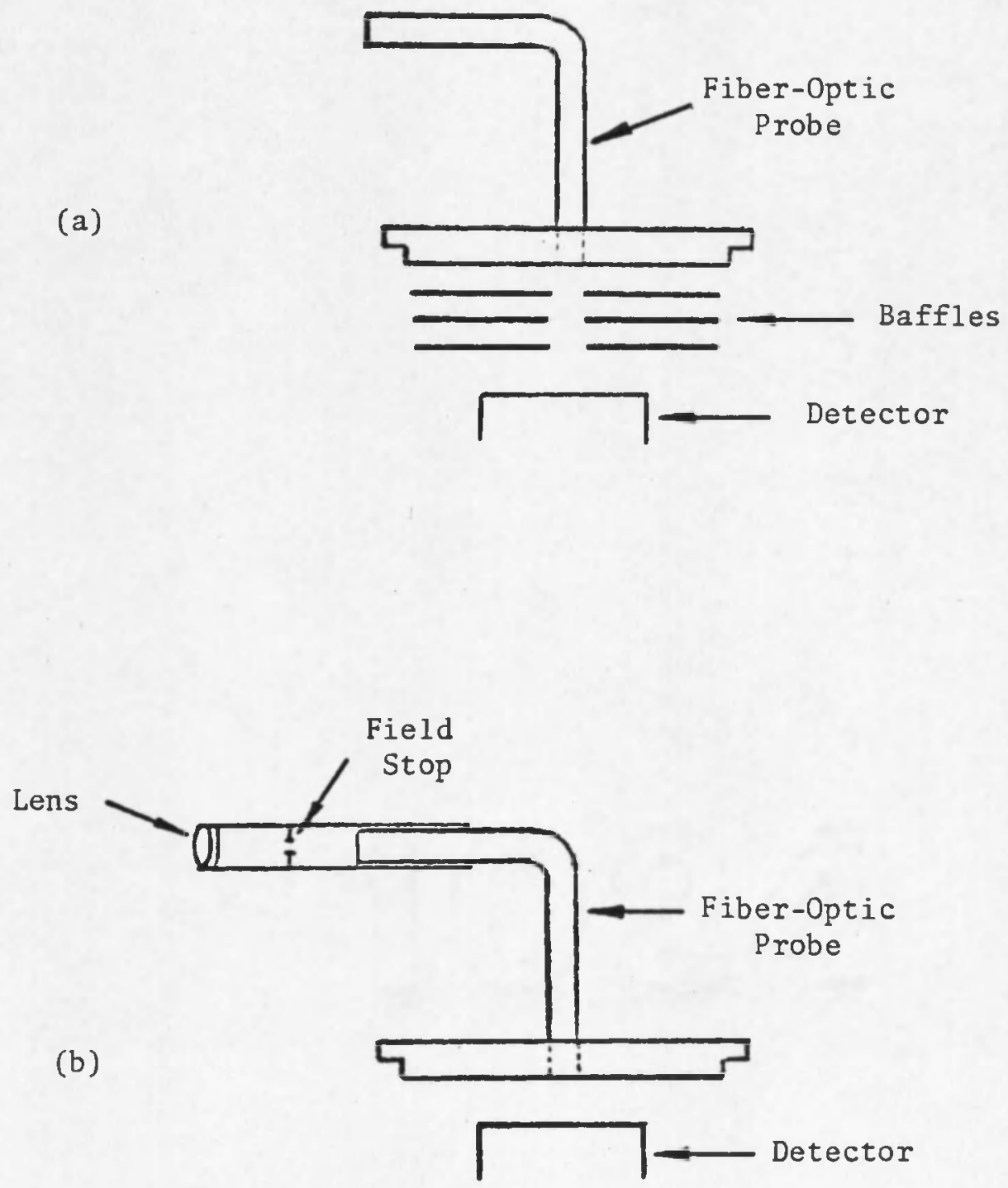

Fig. 4.3. Illustration of Detector Probe Unit.

(a) Previous Configuration.

(b) New Configuration. 
field stop. This is more clearly illustrated in Fig. 4.4. A coated doublet with a 10-mm focal length was edged down and mounted in a black anodized brass tube 3 -mm in diameter. A field stop allowing a 5-degree field of view was fabricated and inserted into the tube at the rear focal plane of the lens. This assembly was then positioned onto the end of the fiber-optic bundle. The detector response from a small (point source) light source was then recorded as a function of field angle for both detector probe configurations. The results are displayed for comparison in Fig. 4.5. Both the flat response and the sharp cutoff obtained with the modified unit are highly desirable features. The flat response promises to eliminate signal variations due to slight misalignment or wobble in the mechanical instrument while scanning over the hemisphere. The ability to keep the field of view small with a very sharp cutoff is essential for stray light rejection.

The scattered light flux from a polished surface varies by several orders of magnitude over the angular range to be measured. Hence the linearity of the PMT was measured using a calibrated neutral density wedge and several known neutral density filters to vary the incident flux. The resulting linearity curve is shown in Fig. 4.6 and indicates a deviation of less than $1 \%$ over a range of five orders of magnitude of the incident flux. 


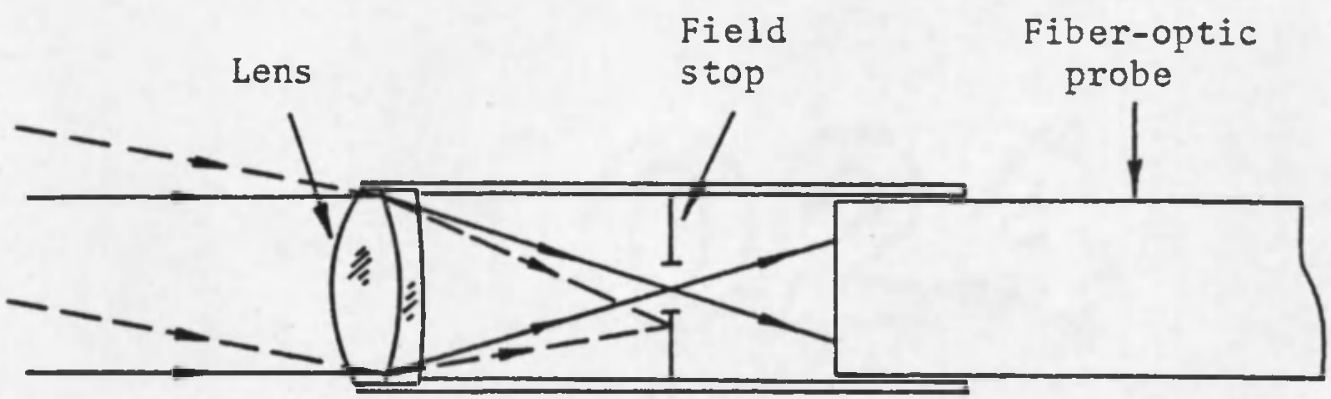

Fig. 4.4. Detailed Illustration of New Fiber-Optic Probe. 


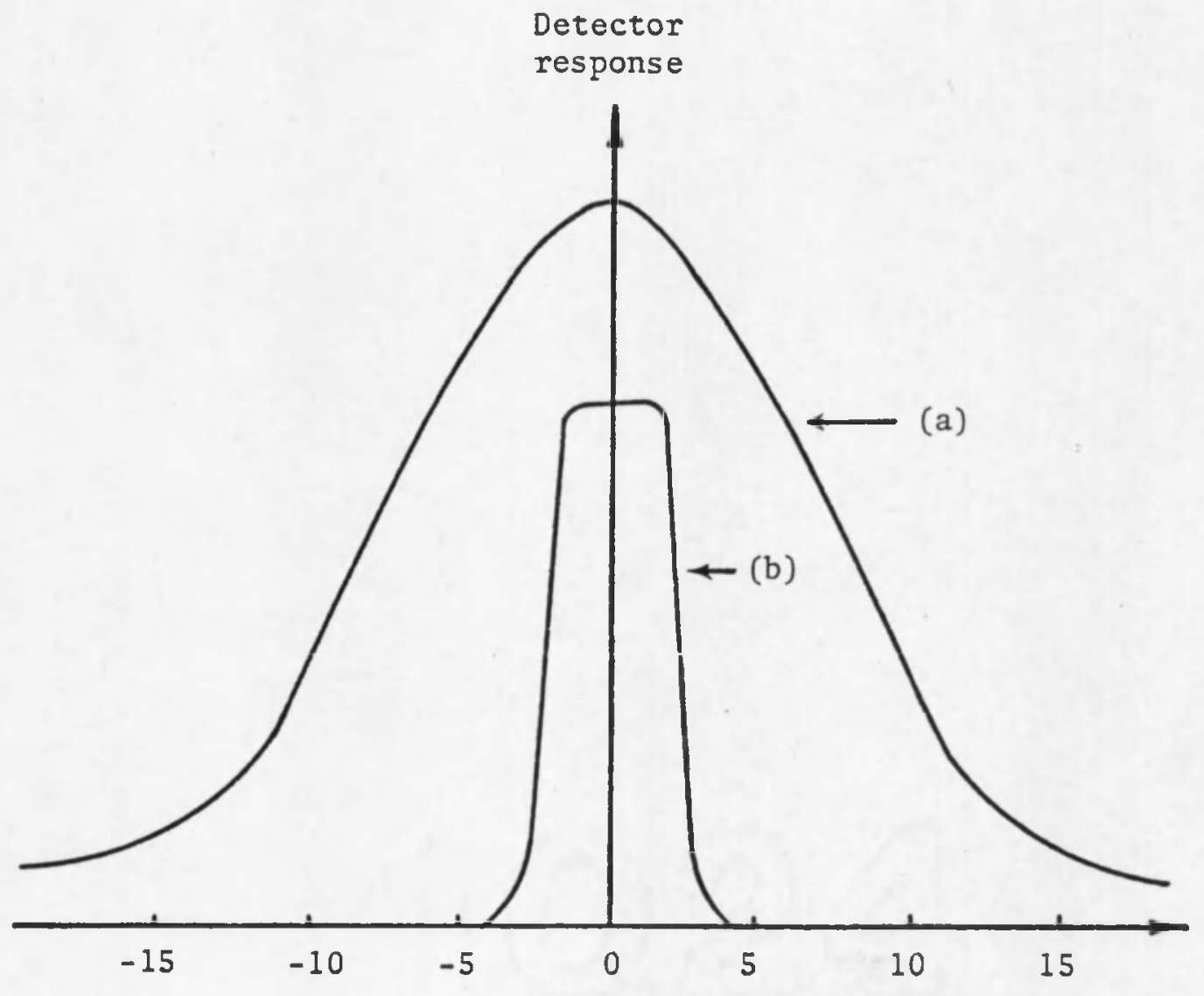

Field angle (degrees)

Fig. 4.5. Detector Response as a Function of Field Angle for Detector Configurations Shown in Fig. 4.3.

(a) Detector configuration shown in Fig. 4.3(a).

(b) Detector configuration shown in Fig. 4.3 (b). 


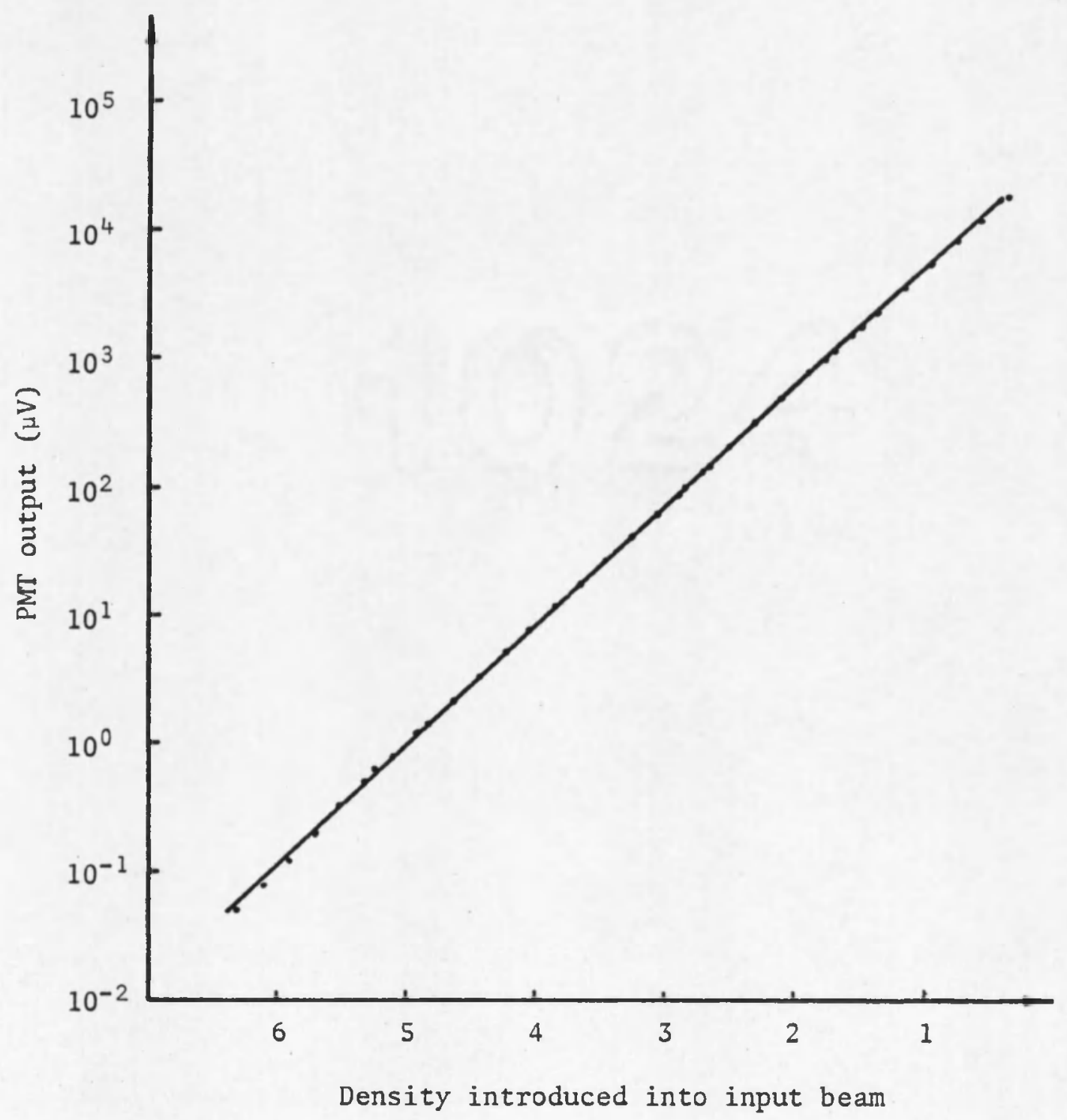

Fig. 4.6. Photomultiplier Tube Linearity Curve. 
The Scanning Mechanism

The mechanical apparatus for measuring the angular distribution of light scattered from optical surfaces was shown in Fig. 4.2. The detector probe unit is mounted on a rigid arm that can be rotated in either of two orthogonal directions. Rotation about a vertical axis is accomplished by means of a massive precision rotary table. The rigid arm is attached to the rotary table by means of a worm gear arrangement that allows rotation about the horizontal axis. These two separate driving mechanisms allow us to measure the scattered light distribution over the entire hemisphere bounded by the plane of the sample. However, in order to limit the quantity of data to be collected, the scattered radiation field is sampled in two principal directions. These include the entire plane of incidence and a plane perpendicular to both the plane of incidence and the plane of the sample which also passes through the intersection of the specular beam with the observation hemisphere (see Fig. 4.7). This particular sampling procedure was chosen because each sampling direction then involves one fixed coordinate in direction cosine space. Furthermore, the apparatus was designed such that each of the two independent drive mechanisms corresponds directly to a given coordinate in direction cosine space. Hence for a given observation point determined by the angles $\theta$ and $\phi$ displayed on the apparatus, the corresponding coordinates in direction cosine space are given by

$$
\begin{aligned}
& \alpha=\cos \theta \sin \phi \\
& \beta=\sin \theta .
\end{aligned}
$$




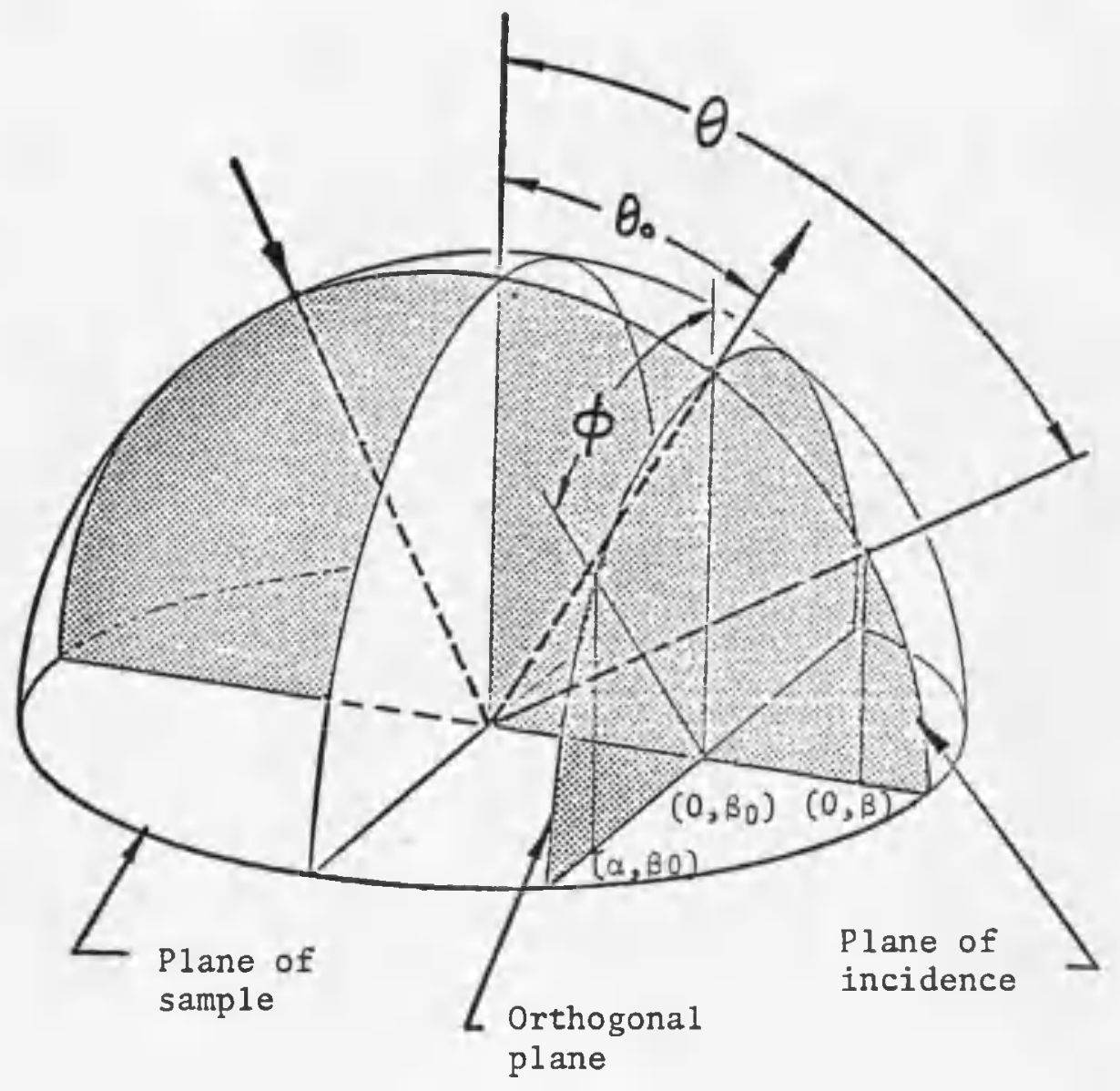

Fig. 4.7. Geometrical Configuration of Two Principal Planes in which the Scattered Light Field was Sampled. 
The Incident Beam

A large movable arm with appropriate folding mirrors is used to direct the incident beam onto the sample at any desired angle of incidence. The original configuration included a single lens positioned on this arm to focus the incident radiation onto the hemisphere mapped out by the detector as shown in Fig. 4.8. This configuration allowed scattered light from the folding mirror M3 to reach the observation hemisphere after being reflected from the test sample. The scattered 1ight leve1 from M3 often exceeded that from the test sample, thus rendering the scattered light data at small observation angles completely useless: (Shack and DeBel1, 1974).

An improved configuration is shown in Fig. 4.9. Immediately following the last folding mirror the beam. is focused onto a pinhole which acts as a spatial filter. This spatial filter assembly eliminates from the beam incident upon the sample any light scattered from the folding mirrors as well as any diffraction effects from the chopper blade. Lens L2 then forms an image of the pinhole upon the hemisphere mapped out by the collecting aperture of the scanning fiber-optic probe. The dramatic improvement obtained with this configuration is illustrated by comparing Fig. 4.10(a) with Fig. 4.10(b). These photographs were obtained by placing a piece of photographic film in the observation space at position B in Fig. 4.9 along with a small obstruction to block the specular beam. The six bright spots in Fig. 4.10(b) were the weld marks on the back side of the pinhole which were illuminated by the light reflected from L2 then imaged by L2 onto the observation space. 


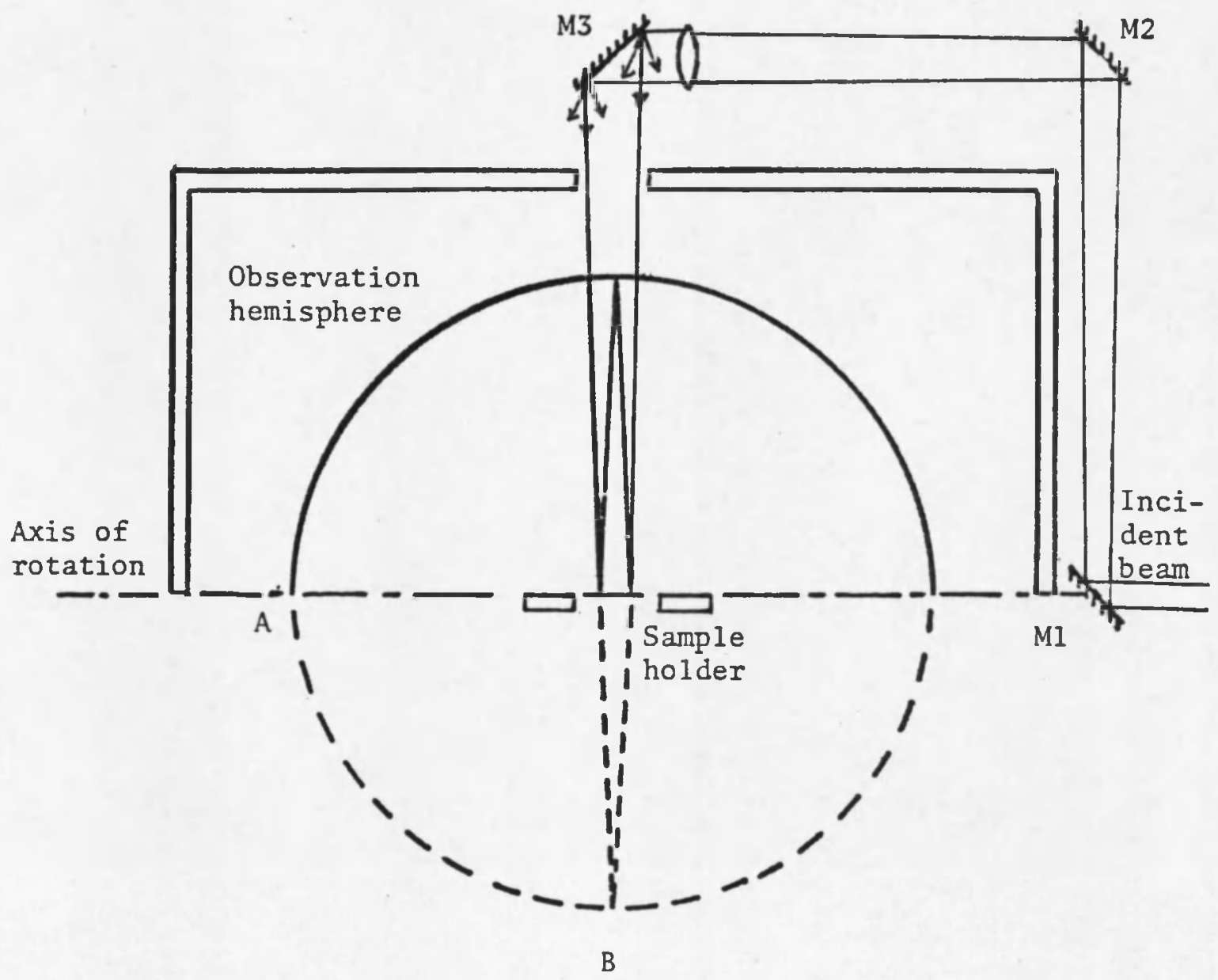

Fig. 4.8. Original Configuration of Beam-forming Optics. 


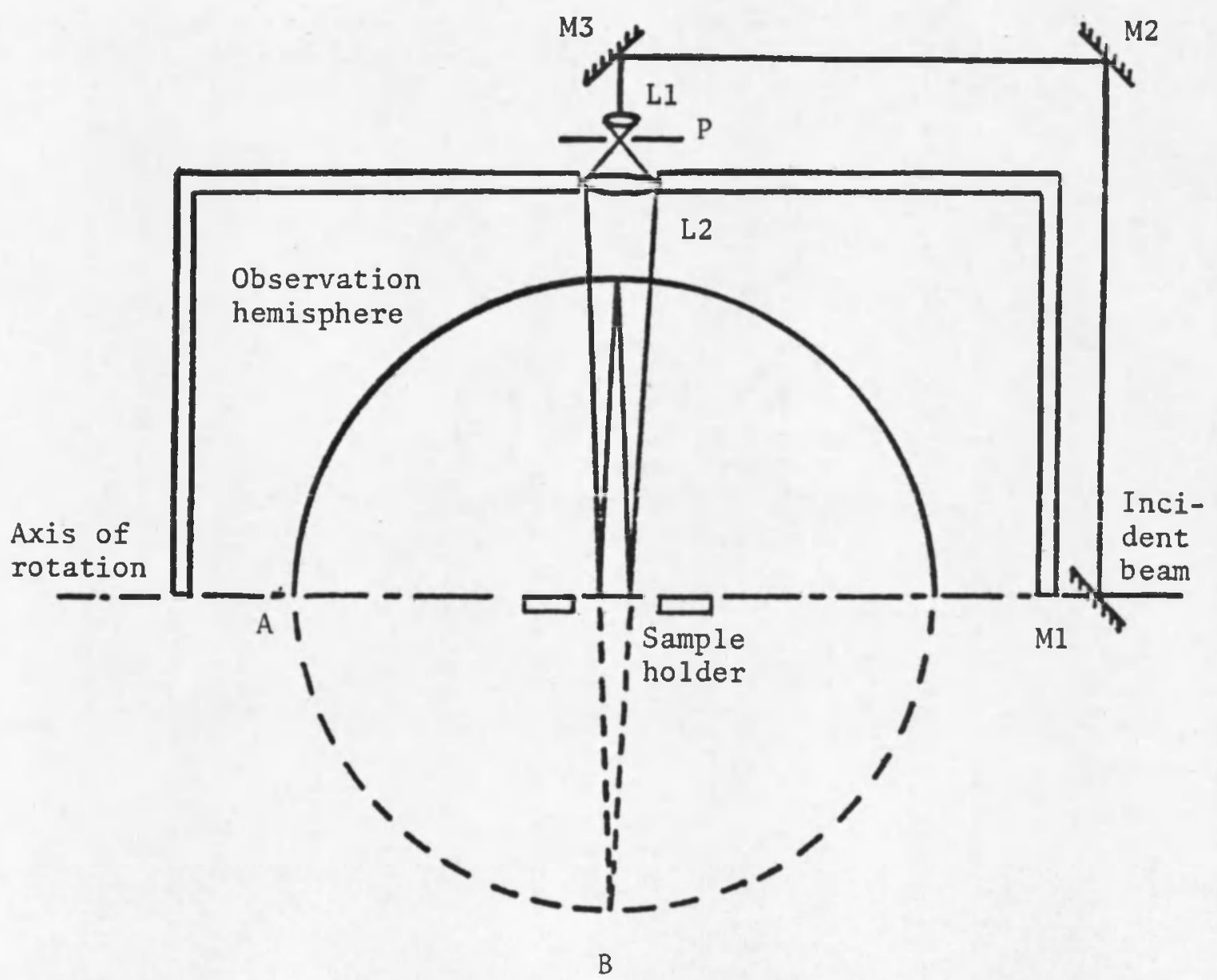

Fig. 4.9. Improved Configuration of Beam-forming Optics. 

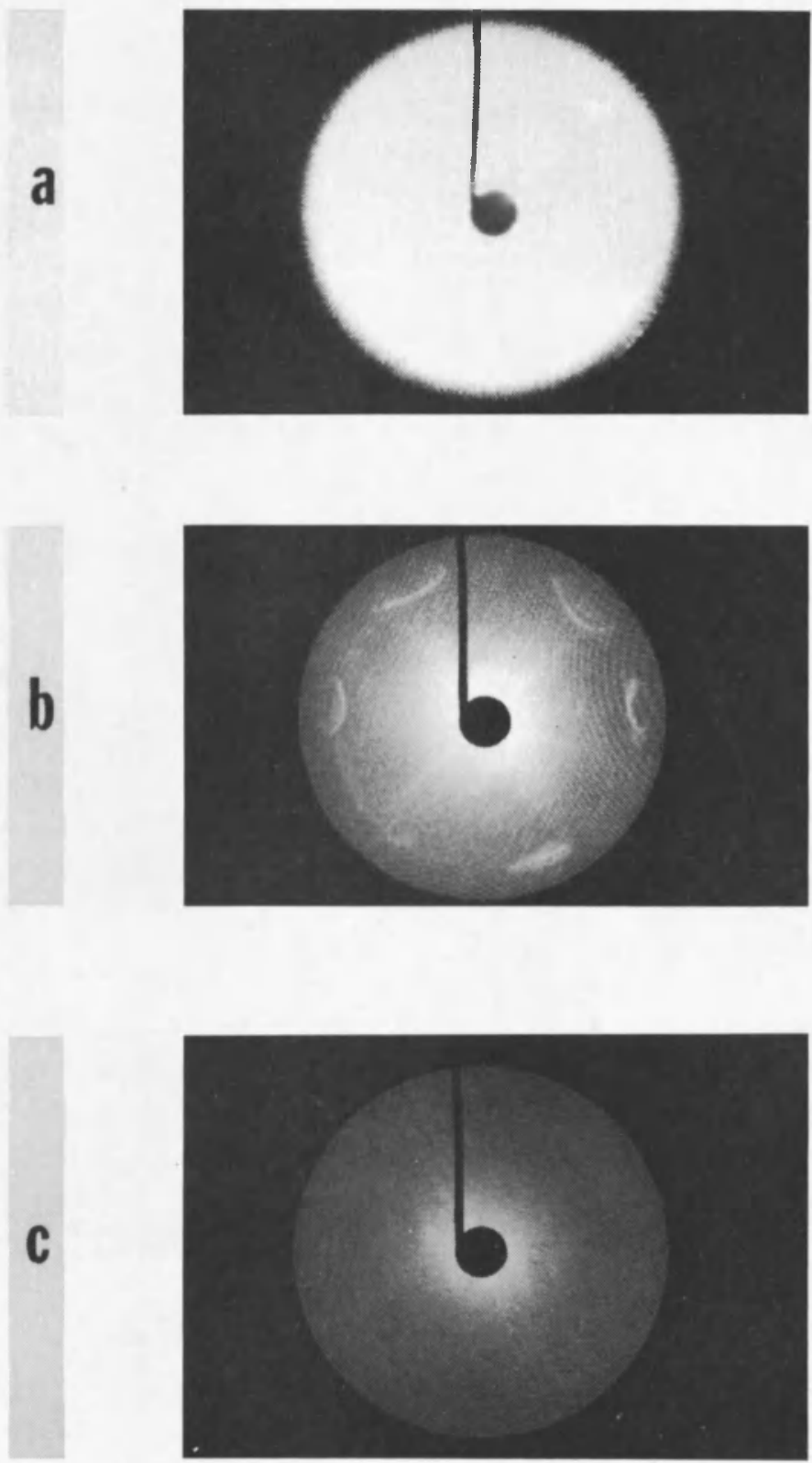

Fig. 4.10. Photograph of Stray Light Reaching the Observation Space for:

(a) Original configuration of beam-forming optics.

(b) Improved configuration of beam-forming optics.

(c) Improved configuration with black pinhole. 
The acquisition of a pinhole in a black substrate with no visible weld joints resulted in the photograph displayed in Fig. 4.10(c). Based upon measurements made with the instrument with no sample in place, the stray light in the observation space has thus been reduced by almost two orders of magnitude.

The aberrations associated with the scattered light distribution on the observation hemisphere were considered in determining the geometrical parameters of the incident beam and the detector scanning mechanism. The aberration coefficients presented in the last column of Table 2.1 yield the following values for coma and astigmatism at the edge of the field $\left(\theta=90^{\circ}\right)$ when a spot $4 \mathrm{~mm}$ in diameter is illuminated on the sample and the observation distance is $250 \mathrm{~mm}$,

$$
\begin{aligned}
& \hat{W}_{131}=0.128 \\
& \hat{W}_{222}=16 .
\end{aligned}
$$

Although a substantial amount of astigmatism exists at the edge of the field, our tolerance is quite loose as we have a $3 \mathrm{~mm}$ diameter collecting aperture on the fiber-optic probe.

\section{Experimental Procedures}

The goal of the research reported here was to examine the scattering properties of samples whose surface characteristics span those typically produced with optical fabrication techniques. 
Sample Preparation

Surface preparation techniques used to produce a set of samples are outlined in Table 4.1. All samples were finished to be nominally flat.

The prepared samples were cleaned prior to coating with aluminum. Cleaning consisted of careful washing with Liquinox, a mild detergent, under very warm, filtered tap water. Samples were then mounted in a sample holder while held in distilled water. Once in a holder, samples were moved to an ultrasonic cleaner filled with distilled water for rinsing. Once rinsed the samples were set to dry in a dust-free "atmosphere. Dry samples were removed from the sample holders and placed in individual boxes and supported by the edge of their backside. Mott (1971) used a similar cleaning technique, which he describes more completely.

Cleaned dry samples were then placed in a high vacuum chamber and coated to near opacity with pure aluminum. The coating technique varied from standard procedures only in that excessive care was taken to allow the chamber to reach a pressure below $2 \times 10^{-6}$ torr prior to coating. The samples were allowed to cool to room temperature prior to removal from the chamber. Each coating run contained ten different samples. Once coated, samples were returned to their individual storage boxes. After al1 samples were coated, the best samples of each type were selected for measurement. This selection was made on the basis of individual inspection of each sample while held under a microscope illuminator in an otherwise dark room. Samples that had coating nonuniformities, sleeks or pinholes were rejected as were those with 
Table 4.1. Surface Preparation Techniques.

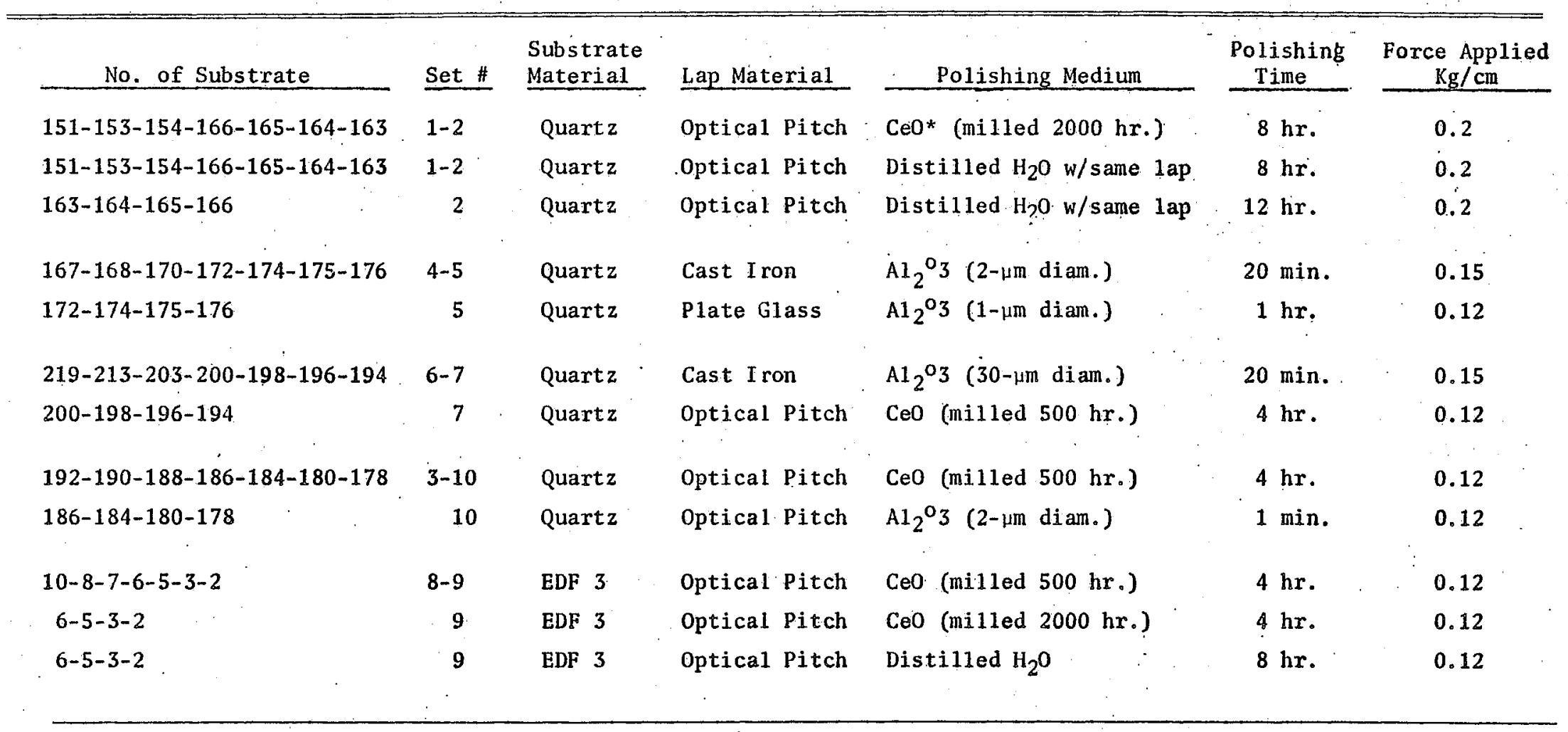

*Closed circulation system. 
waterspots, large scratches, or otherwise questionable appearance. Prior to each set of scatter measurements, samples were again individually inspected for flaws. Dust was removed using a commercially available pressurized air can. After each sample was measured for scatter, it was returned to its individual box.

System Alignment

Before any meaningful scatter measurements could be made it was necessary to systematically align the entire system.

The incident laser beam was first adjusted to 1ie in a horizontal plane. Then the mechanical apparatus was positioned such that the axis of rotation of the movable arm supporting the folding mirrors (see Fig. 4.9) was colinear with the incident beam. This was accomplished by means of four massive leveling screws at the base of the stand supporting the entire apparatus.

With mirror M1 removed the laser beam passed through the smal1 hole in the center of the bearings upon which the movable arm rotates. This assured that the incident beam was indeed colinear with the axis of rotation and furthermore allowed the sample holder, which was mounted on a shaft passing through the axis of the precision rotary tablè, to be accurately positioned with the center of the scattering surface lying at the intersection of these two perpendicular axes. The precision rotary table was then positioned such that the beam was accurately centered on the detector at position $A$, then rotated precisely $90^{\circ}$, leaving the detector at position $B$. 
Mirror M1 was then put into place and adjusted until the laser beam was centered upon mirror M2. Similarly, M2 was adjusted until the beam was centered upon $\mathrm{M} 3$.

With the lenses $\mathrm{L} 1$ and $\mathrm{L} 2$ and the pinhole $\mathrm{P}$ removed from the system, mirror M3 and mirror M2 were systematically adjusted until the beam was centered upon both the sample holder and the detector at position $B$.

The incident beam was thus accurately positioned perpendicular to the axis of rotation of the movable arm. A polished sample was then placed in the holder and adjusted until the specularly-reflected beam returned precisely along the incident beam. The sample holder was thus accurately positioned perpendicular to the incident beam and locked into this position...

Lenses L1 and L2 were then placed in the beam and properly centered. And finally, the pinhole $P$ was accurately positioned at the back focal position of lens L1.

With the system properly aligned, the movable arm could be rotated to direct the incident beam at any desired angle without requiring other adjustments to keep the beam centered upon the sample.

An additional requirement was that the PMT with its associated fiber-optic probe be positioned and aligned such that the field of view of the PMT remain accurately centered on the illuminated portion of the sample throughout the entire range of its scanning motion. Provisions were therefore made to allow three degrees of freedom (one translation and two rotation) in adjusting the position and orientation of the PMT 
housing. Removing the PMT from its housing and illuminating the fiberoptic probe from the back side greatly facilitated this alignment procedure as it allowed one to directly observe the field of view on the sample holder while making the necessary adjustments.

Measurement Technique

The sample to be measured was placed in the holder and the movable arm positioned to achieve the desired angle of incidence. A calibrated attenuator was then placed in the incident beam and the detector centered on the specularly-reflected beam. The collecting aperture of the fiber-optic probe was large enough to collect the entire specular beam; hence, the output signal, $V_{S}$, of the PMT in this position was proportional to the total flux in the specular beam.

The detector was then moved a known angular distance (approximately one degree) from the specular beam and the attenuator removed. A profile of the scattered light distribution was then measured by scanning the observation hemisphere with the fiber-optic probe. Approximately 30 separate readings were taken at different angular positions between the specular beam and the plane of the sample. These readings constitute the raw data.

The sample was then removed and the incident beam allowed to pass unobstructed through the sample holder and into a black absorbing Rayleigh horn. Background measurements were then made along the same profile as above and subtracted from the raw data. These background measurements were found to be completely negligible in most instances. 
The data now represents the spread function of the scattering system, which is made up of the spread function of the scattering surface convolved with the spread function of the optical system producing the incident beam. These are shown in Fig. 4:11.

The spread function of the incident beam is then measured by again placing the calibrated attenuator in the incident beam and centering the detector on the direct beam passing through the empty sample holder. Since the collecting aperture of the fiber-optic probe is large enough to collect the entire incident beam, the output signal, $V_{o}$, of the PMT in this position is proportional to the total flux in the incident beam. The detector is then moved a known angular distance (approximately one degree) from the direct beam and the attenuator removed. A profile of the incident beam is then measured. These readings rapidly diminish to zero within five degrees of the peak value. Since the spread function of the incident beam [Fig. 4.11(a)] is narrow compared to the scattering function of the surface [Fig. 4.11(b)], the scattered portion of the surface spread function is virtually unchanged by the convolution operation while the delta function component merely replicates the beam spread function. The desired scattering function can thus be obtained by subtracting the beam spread function readings from the raw data.

\section{Presentation of the Data}

It is customary to present scattered light data from diffusely reflecting surfaces in a polar format. Three experimental curves and one ideal reference curve are illustrated in Fig. 4.12. Note that we 

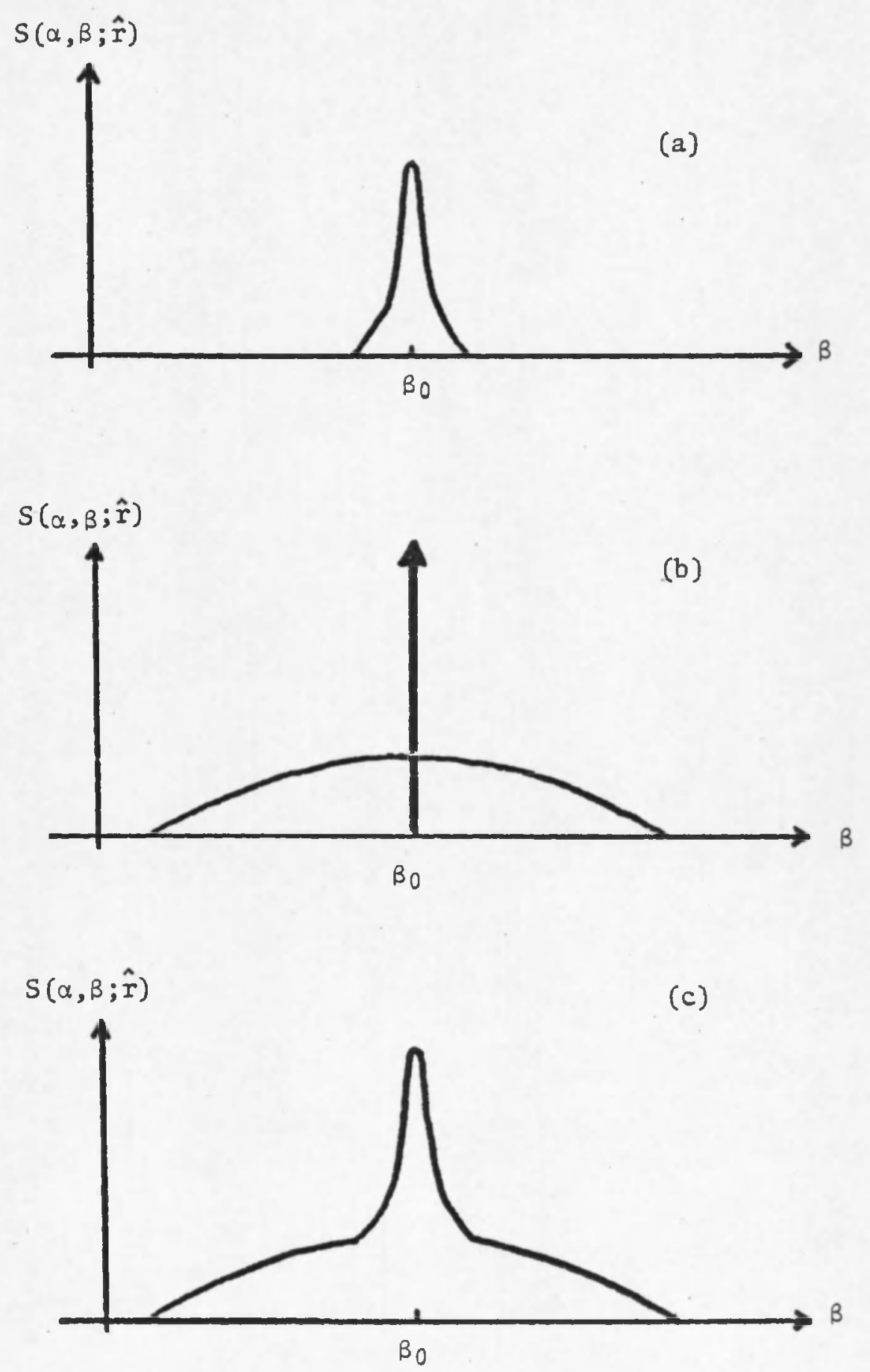

Fig. 4.11. I1lustration of Components Comprising System Spread Function.

(a) Spread Function of Incident Beam.

(b) Spread Function of Scattering Surface.

(c) Spread Function of Scattering System. 


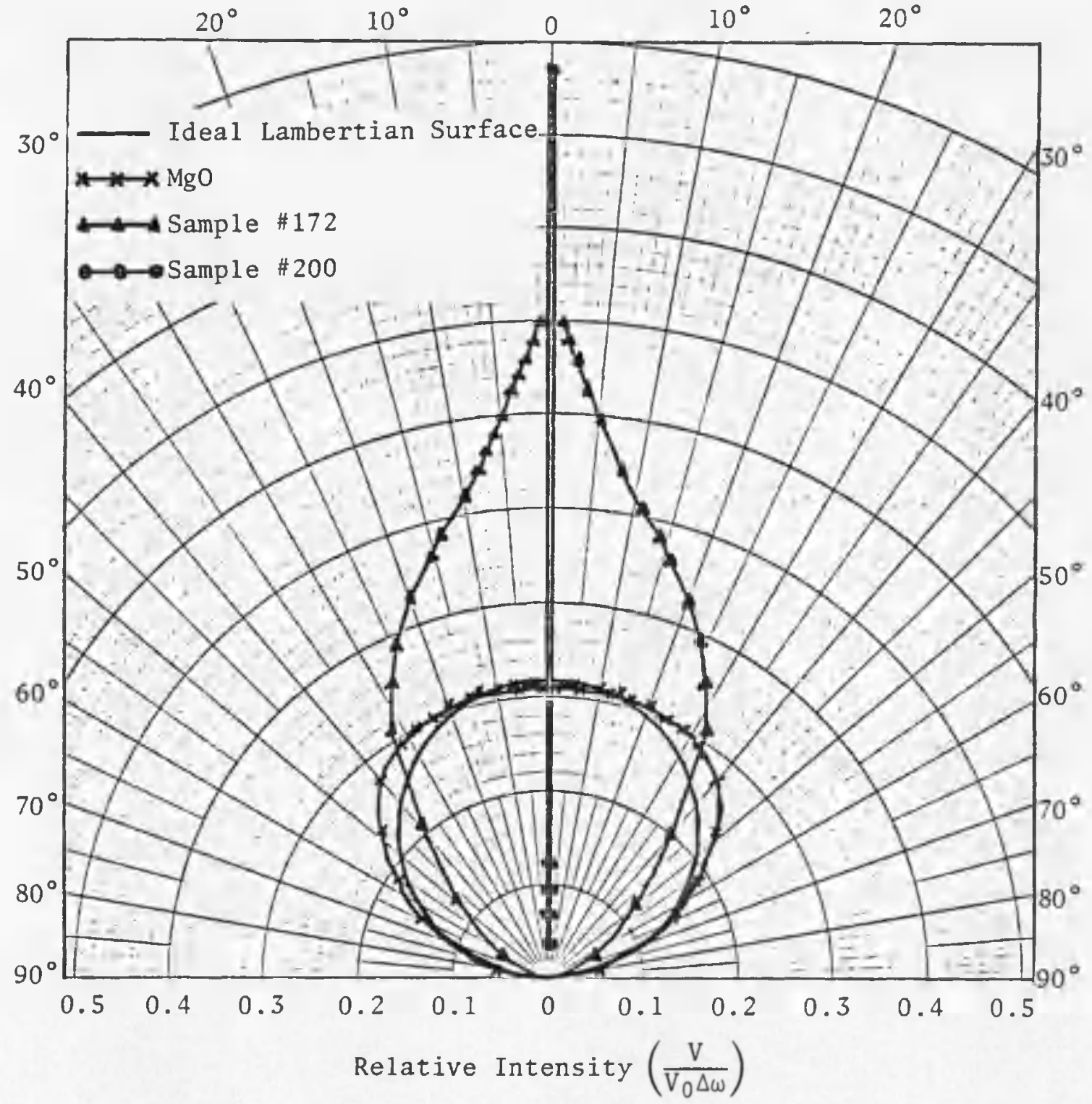

Fig. 4.12. Polar Plot of Relative Intensity as a Function of Scattering Angle. 
have plotted the quantity $\frac{V}{V_{0} \Delta \omega}$ along the radial coordinates where $\Delta \omega$ is the solid angle subtended by the fiber-optic probe. Since $V$ is proportional to the power collected by the fiber-optic probe and $V_{0}$ is proportional to the total power in the incident beam, this quantity is the relative intensity of the scattered light distribution (i.e., scattered intensity normalized by the incident power). For smooth mirror surfaces this same quantity is usually plotted in a cartesian format as a function of the scattering angle as shown in Fig. 4.13. Dividing this data by the cosine of the scattering angle $(\gamma=\cos \theta)$, we obtain

$$
\frac{V}{V_{0} \Delta \omega \gamma}=\frac{P /(A \gamma \Delta \omega)}{P_{0} / A}=\frac{L_{r}}{E_{i}}
$$

where $A$ is the illuminated area on the sample. This quantity is equivalent to the reflected radiance in the sampled direction divided by the incident irradiance, which is precisely the manner in which the BRDF is defined. The resulting scattering curve, exhibited in Fig. 4.14, is therefore a one-dimensional profile of the four-dimensional BRDF. Also, in accordance with our theory we are plotting this function versus the quantity $\beta-\beta_{0}$, which is the distance of the observation point from the specular beam in direction cosine space.

It can be readily shown that the BRDF is merely an infinite family of two-dimensional spread functions which are scaled by the total reflectance of the surface, 


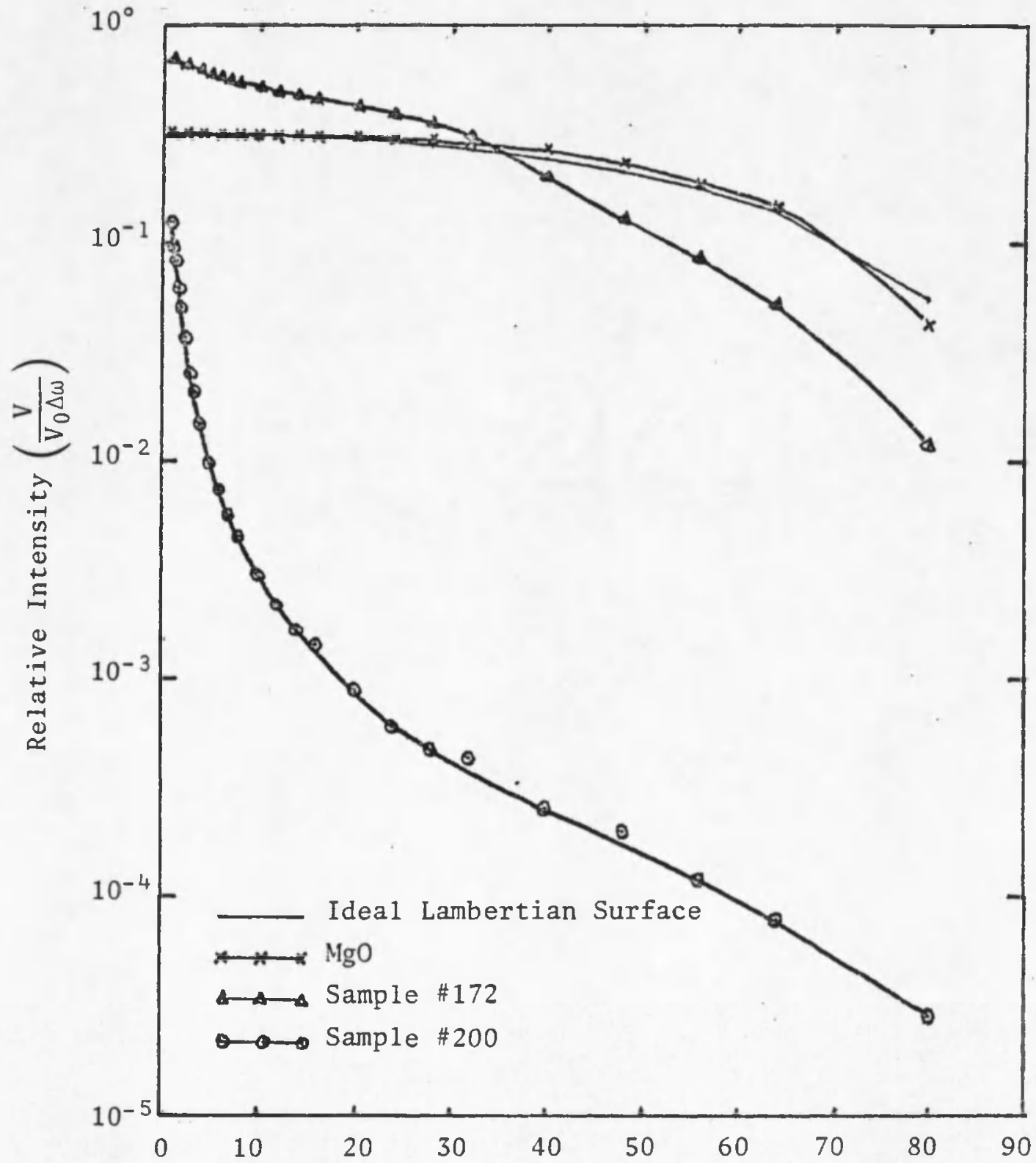

Fig. 4.13. Illustration of Relative Intensity Plotted as a Function of Scattering Angle. 


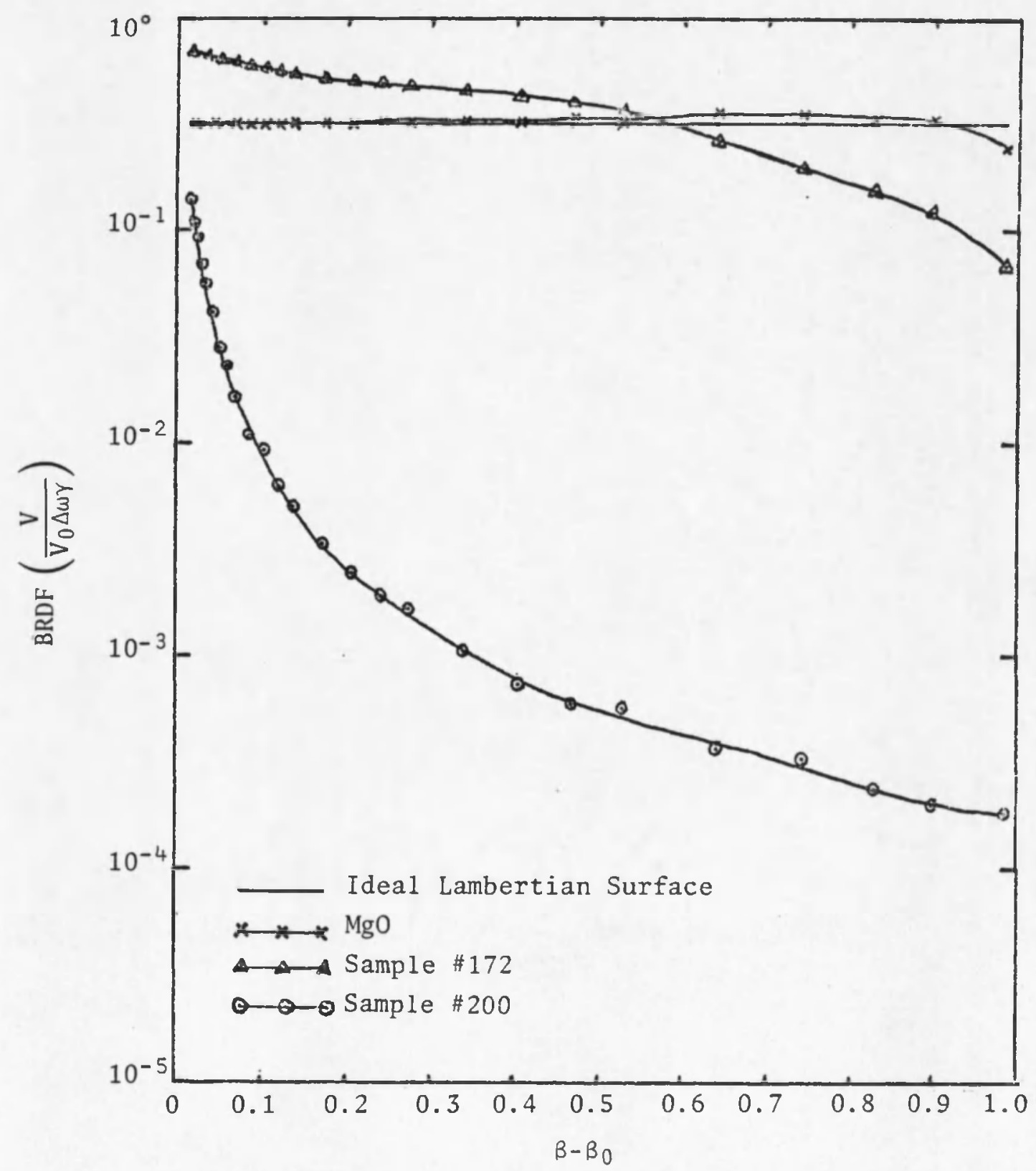

Fig. 4.14. Illustration of BRDF Profiles Plotted as a Function of $\beta-\beta_{0}$. 


$$
\mathrm{BRDF}=\sum_{\theta_{i \phi_{i}}} R \mathscr{Q}(\alpha, \beta)
$$

Since we measure only the scattered component of the spread function (See Eq. 57) and are primarily concerned with the scattered light behavior rather than the total reflectance, the scattering function. can be written as

$$
S(\alpha, \beta)=\frac{V(\alpha, \beta)}{R V_{O} \Delta \omega \gamma} .
$$

A11 measurements are thus normalized by the reflectance of the surface so it does not appear to have better scattering characteristics due to its lower reflectance.

The MgO surface, which is a fairly good diffuse reflectance standard, yields a straight horizontal line as a Lambertian reflector should. Since the radiance of a Lambertian source is given by

$$
L=\frac{M}{\pi}
$$

where $M$ is the total emittance into a hemisphere, the value of the MgO scattering function can be shown to be $\frac{1}{\pi}$. A Lambertian surface of known reflectance, $R_{L}$, thus makes a convenient reference sample and the scattering function of a test sample of reflectance, $R$, is given by

$$
S(\alpha, \beta)=\frac{V(\alpha, \beta) R_{\ddot{L}}}{R V_{L} \pi \gamma}
$$

where $V_{L}$ is the PMT voltage signal from the Lambertian reference surface at $\alpha=\beta=0$. A freshly coated MgO sample with a tota1. - 
hemispherical reflectance $R_{L}=0.98$ was routinely used as a reference sample for the remainder of the measurements. Unless stated otherwise, the scattering function of Eq. (77) will be used for presenting the data in this dissertation. Furthermore, in accordance with the theory presented in Chapter 2, this scattering function will be plotted as a function of distance from the specular beam in direction cosine space $\left(\beta-\beta_{0}\right)$ as shown in Fig. 4.15. Note that by plotting both ordinate and abscissa on a $\log$ scale the scattering function for the polished sample takes the form of a straight line. 


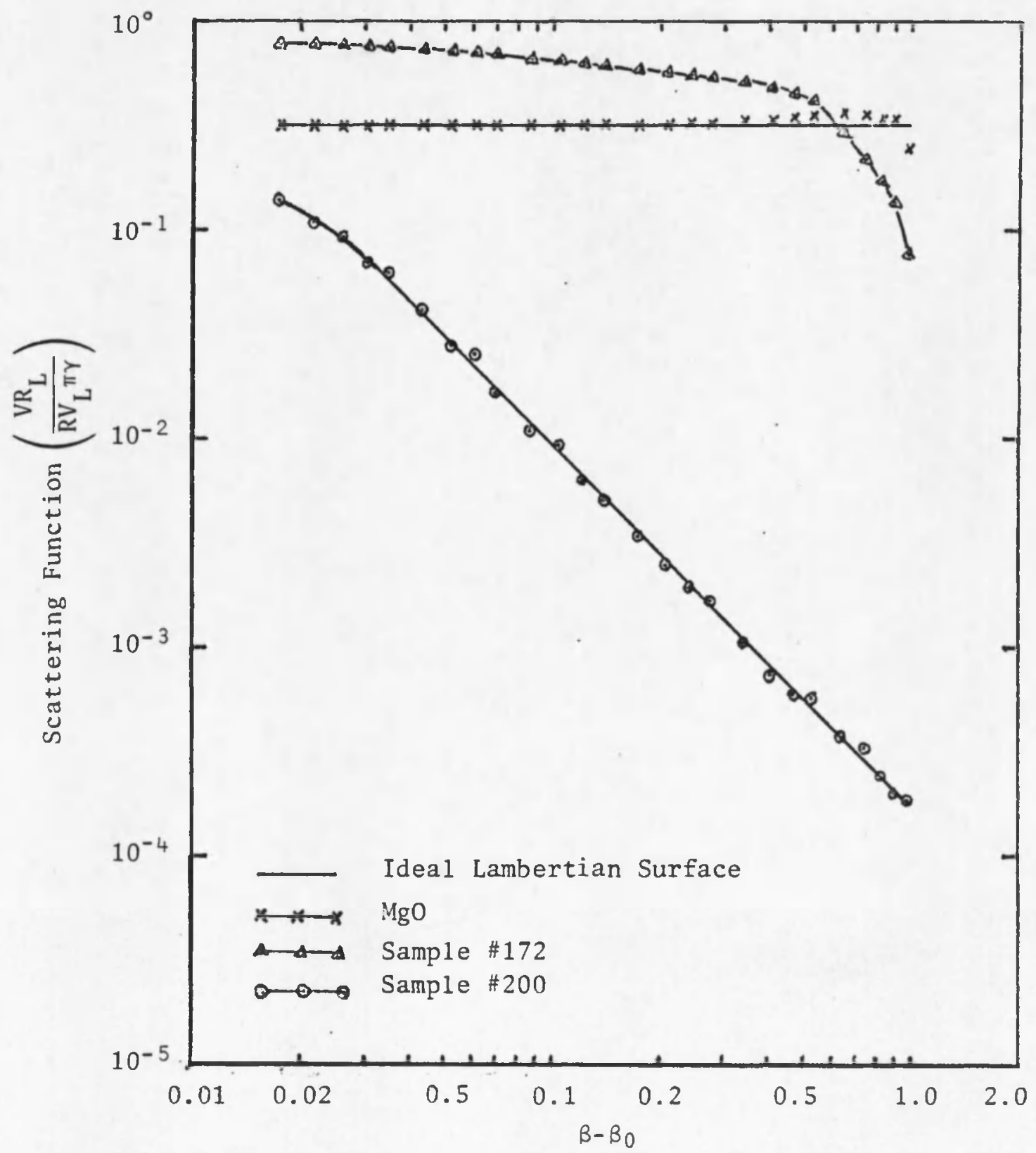

Fig. 4.15. Illustration of Scattering Function Plotted as a Function of $\beta-\beta_{0}$. 
CHAPTER 5

DATA AND RESULTS

The angular distribution of scattered light measured from a variety of surfaces, angles of incidence, and wavelengths are reported in this chapter. The results obtained are then compared with theoretical predictions.

\section{Surface Roughness Effects}

The scattered light profile for a normally incident beam on a variety of samples with a wide range of rms surface roughness values are exhibited in Fig. 5.1. Note that the polished samples are characterized by a straight line curve with a slope between $-3 / 2$ and -2 . The ground glass samples yield curves which are flat for a substantial angular range before falling off rapidly at the larger angles. We were unable to produce samples that satisfactorily bridged the obvious gap in the data between the ground and polished samples. A separate study of the grinding and polishing process on fused silica (Shevlin, 1974) utilizing electron microscopic examination of the surface also indicates a very rapid change in the surface character between the fine grind and the polishing operation. This is dramatically illustrated in Fig. 5.2. 


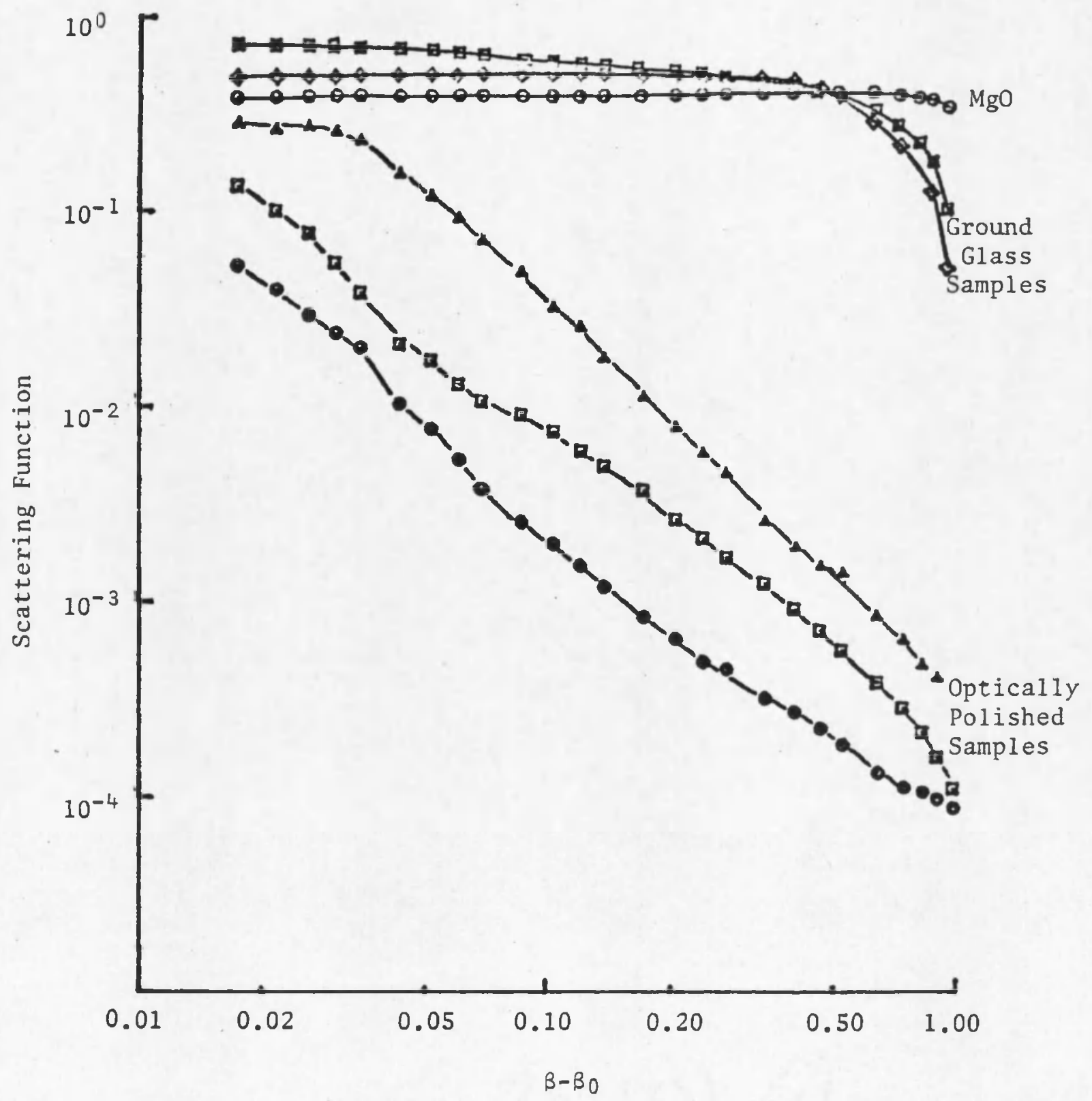

Fig. 5.1. Comparison of Scatter Profiles for Samples with a Wide Range of Surface Roughnesses for a Normally Incident Beam. 


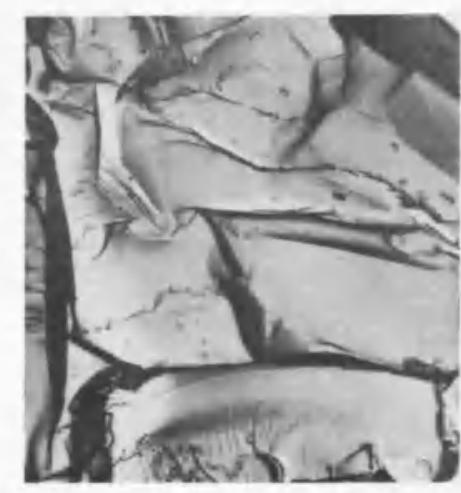

220 Micron SiC Grind $\mathrm{X} 4000$

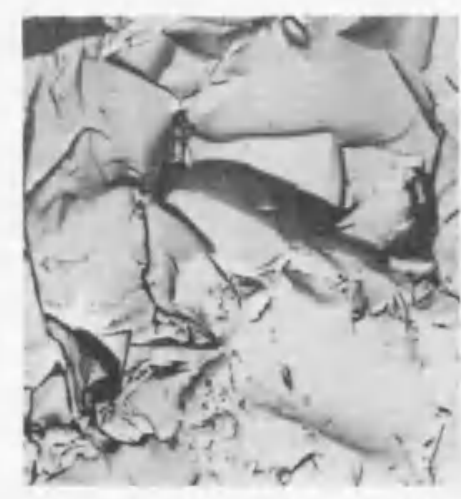

30 Micron SiC Grind

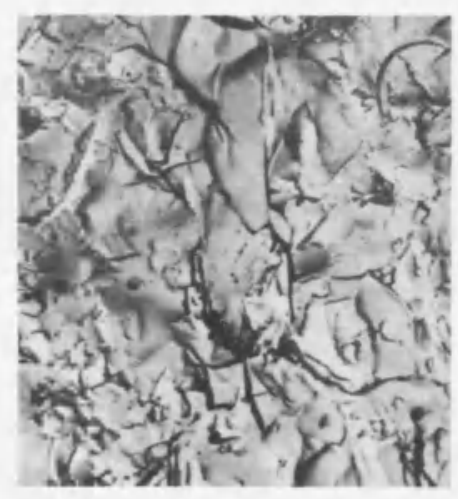

12 Micron SiC Grind

$\times 4000$

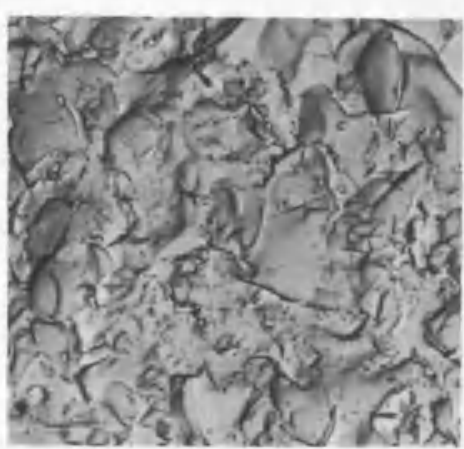

3 Micron SiC Grind $\quad \times 4000$

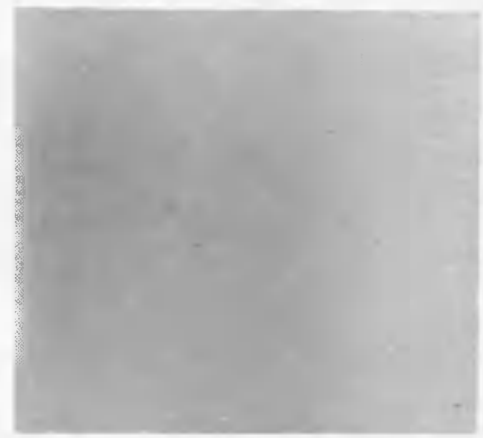

Final Polish - Milled CeO X20,000

Fig. 5.2. Electron Micrographic Display Illustrating the Surface Structure of Fused Silica at Various Stages of the Grinding and Polishing Process. 
It is also instructive to compare the scattering curves resulting from special materials or unusual fabrication techniques with those of more conventional optical surfaces. For example, the results of scattering measurements on a polished beryllium sample and an ionically-polished fused quartz sample are shown in Fig. 5.3 along with some curves from conventional optical surfaces.

\section{Incident Angle Effects}

Rather extensive scatter measurements have been made on two representative surfaces. One is a ground glass surface (Sample \#172) which is a very diffuse reflector. The other is an optically-polished surface (Sample \#200) which is a nice specular reflector. Both samples were coated with aluminum prior to making the measurements. Four separate scattering profiles from the specular bean to the plane of the sample (see Fig. 4.7) were measured at several angles of incidence.

The backscattering profile of the scattered light distribution for these two samples is shown in Fig. 5.4 for several different angles of incidence. For the polished sample, the various curves coincide almost perfectly for angles of incidence between zero and $60^{\circ}$. Hence, it is apparent that the scattering function does not appreciably change with the angle of incidence. The corresponding curves for the rough sample coincide for a substantial range of angles then begin to depart somewhat at the large angles.

The four separate profiles of the seattered light distribution from the same two samples with the incident beam at $45^{\circ}$ are shown in 


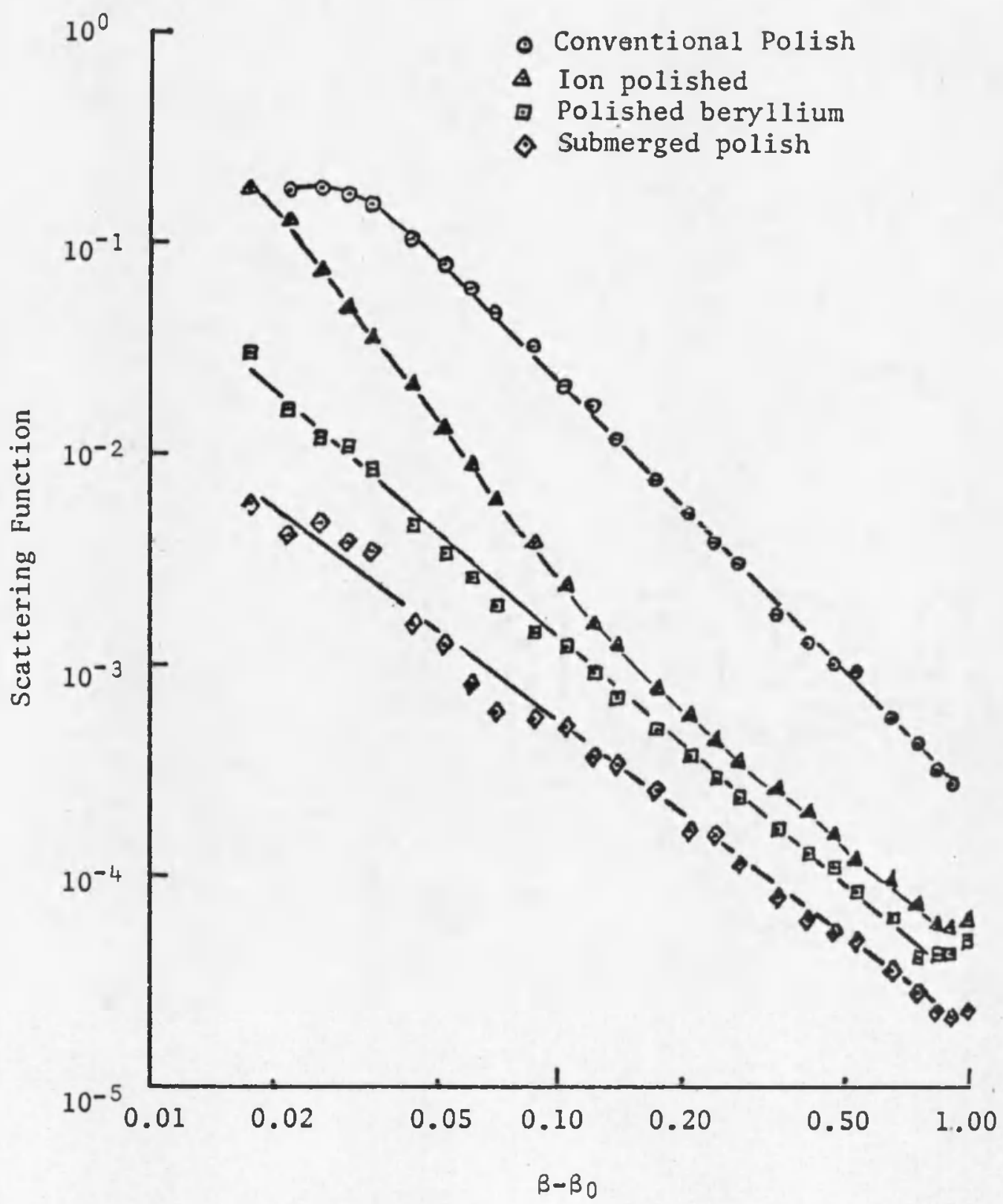

Fig. 5.3. Comparison of Scatter Profiles from Special Materials and Unusual Fabrication Techniques with Those of Conventional Optical Surfaces. 


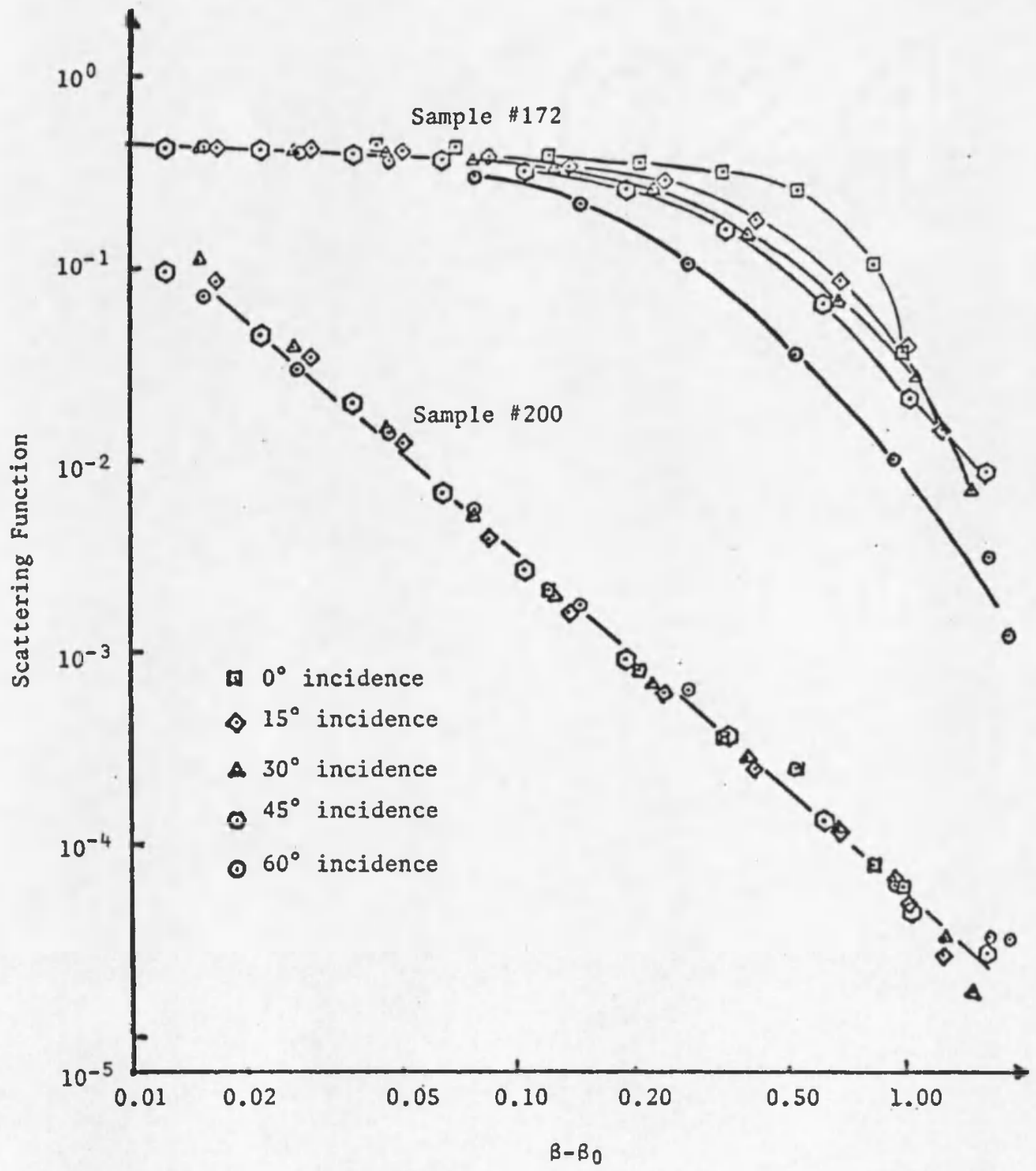

Fig. 5.4. Comparison of Scatter Profiles for Different Incident Angles. 
Fig. 5.5. Again the curves for the polished sample coincide almost perfectly, suggesting a rotationally-symmetric distribution in direction cosine space. Some asymmetry is noted in the scattered light distribution from the rough sample.

The data on Figs. 5.4 and 5.5 confirm that for a certain class of surfaces (in which optically-polished glass is definitely a member, and ground glass can perhaps be included to a lesser extent), the scattering properties are indeed shift invariant as predicted by the theory presented in Chapter 3, and can be completely characterized by a single set of measurements at a fixed angle of incidence!

Figure 5.6 dramatically illustrates the importance of the coordinate system within which the scattering process is discussed. The curves in Fig. 5.6(a) correspond to the scattered light distribution illustrated in Fig. 3.4 and confirms the well-known fact that a curve representing the scattered intensity as a function of observation angle will change shape drastically with angle of incidence--becoming quite skewed and asymmetrical at large angles of incidence. However, these same data, when plotted in accordance with our theory, describes a new scattering function which does not change shape but will merely be shifted in direction cosine space with changes in angle of incidence as shown in Fig. 5.6(b). This is a rather significant development which greatly reduces the quantity of data required to completely characterize the scattering properties of a surface. 


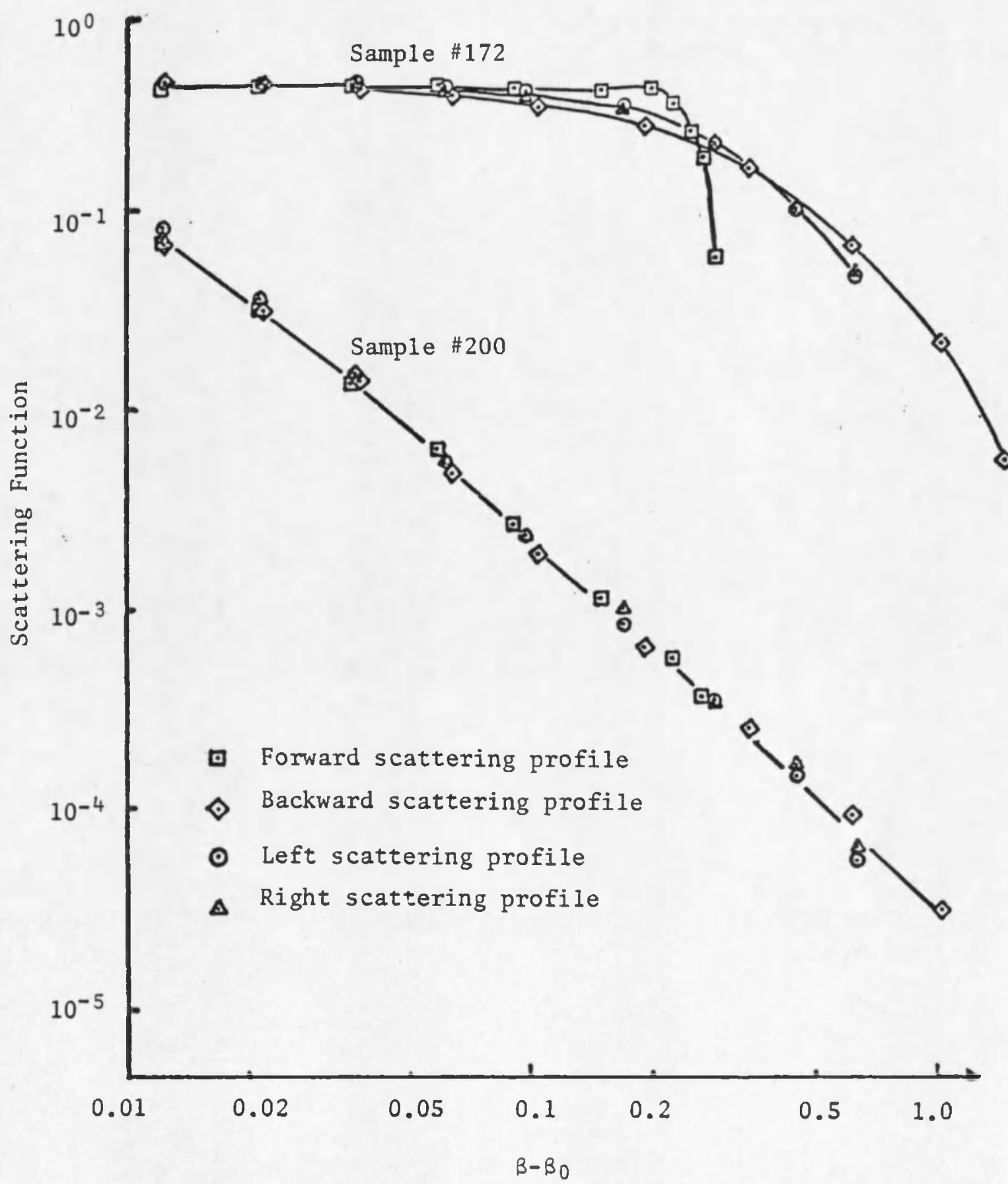

Fig. 5.5. Comparison of Scatter Profiles Taken in Different Directions for an Incident Beam at 45 Degrees. 


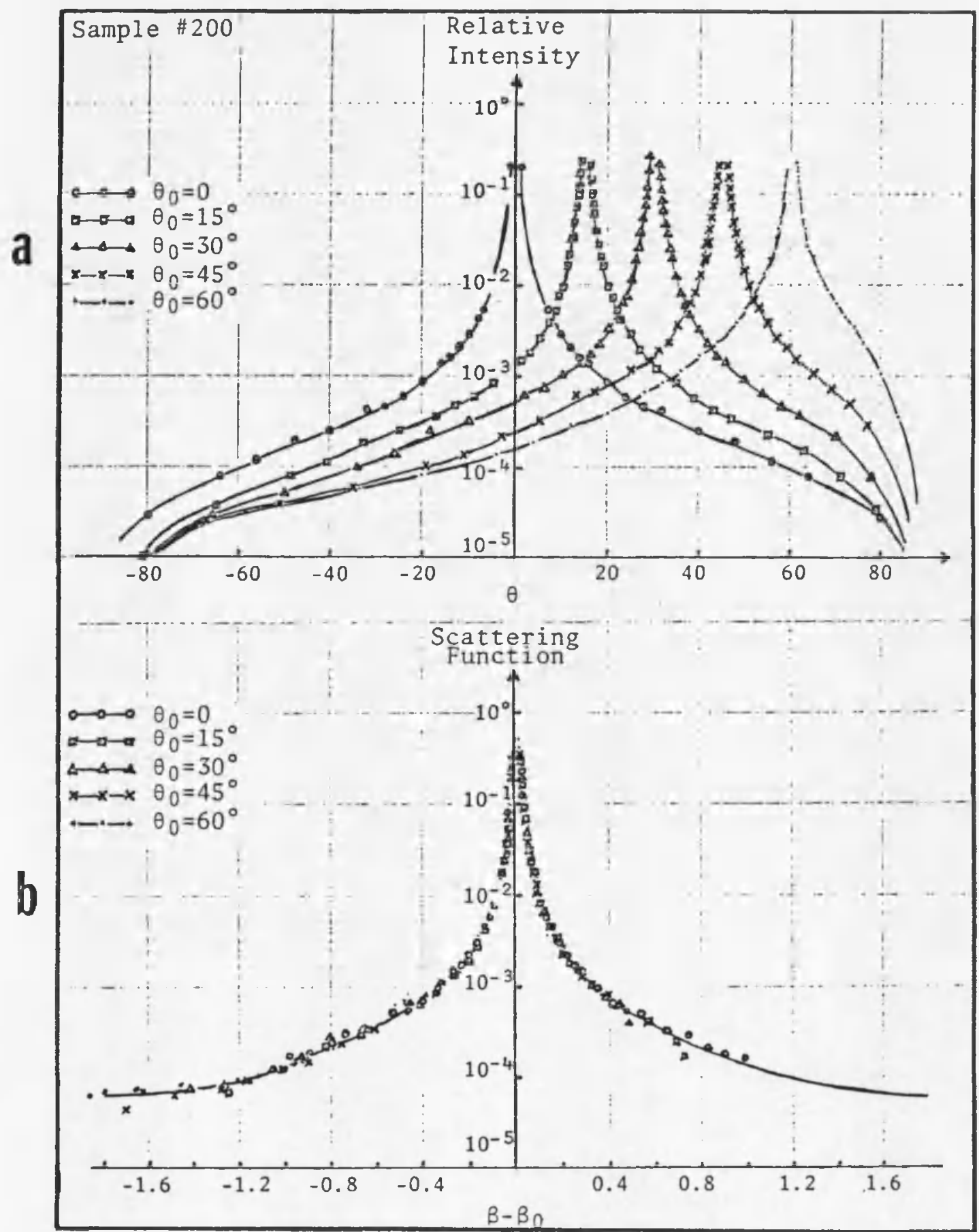

Fig. 5.6. Illustration of the Importance of the Coordinate System within which the Scattering Process is Discussed.

(a) Relative intensity plotted versus scattering angle.

(b) Scattering function plotted versus $\beta-\beta_{0}$. 


\section{The Inverse Scattering Problem}

The problem of determining surface characteristics from scattered light measurements plays an important role in many areas of technical interest. Recall that the surface autocovariance function and the rms surface roughness are two relevant surface characteristics which are related to the scattering properties of the surface by the transfer function described in Chapter 3.

Predictions of Surface Characteristics

A computer program has been written (see Appendix C) for calculating the effective transfer function and the surface autocovariance function from scattered light data. The measured data are assumed to be a radial profile, $S(\rho)$, of a rotationally-symmetric scattering function. An intermediate quantity, $B Q(\hat{s})$, is first determined by calculating the two-dimensional Fourier-Bessel Transform of this scattering function. The surface transfer function is then calculated from Eq. (53), where $A=1-B$. And finally, the surface autocovariance function is computed from Eq. (58). Representative curves for a diffusely reflecting ground glass surface and a specularly reflecting polished glass surface are shown in Fig. 5.7 and Fig. 5.8. Note that the total integrated scatter, $B$, and the rms surface roughness, $\sigma_{W}$, are also provided from these curves. Figures 5.9 through 5.13 illustrate the predicted surface characteristics from other samples which we wish to compare with independent measurements. 

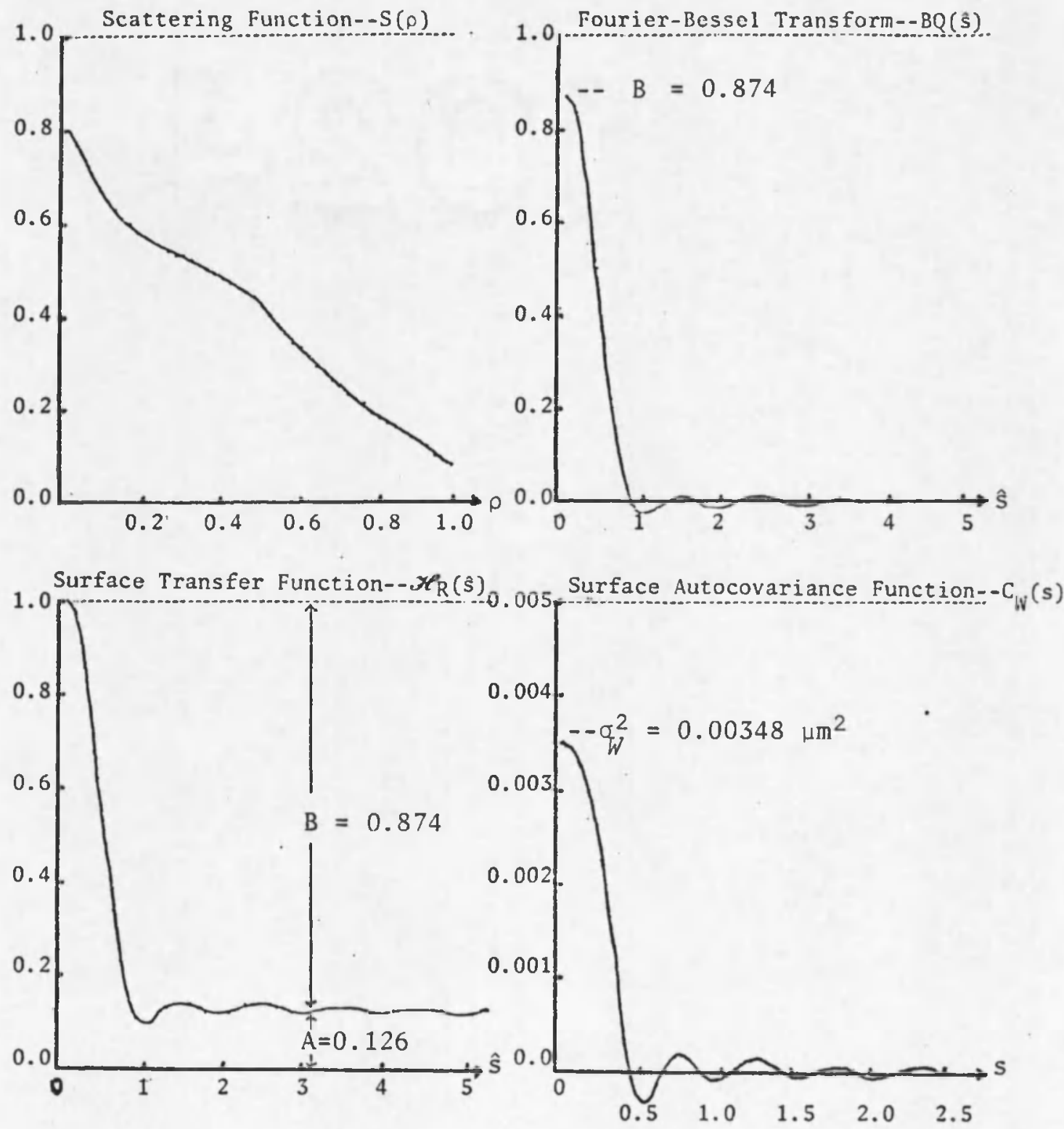

Fig. 5.7. Computer Output from Inverse Scattering Program for Sample \#172 $(\lambda=0.5145 \mu \mathrm{m})$. 

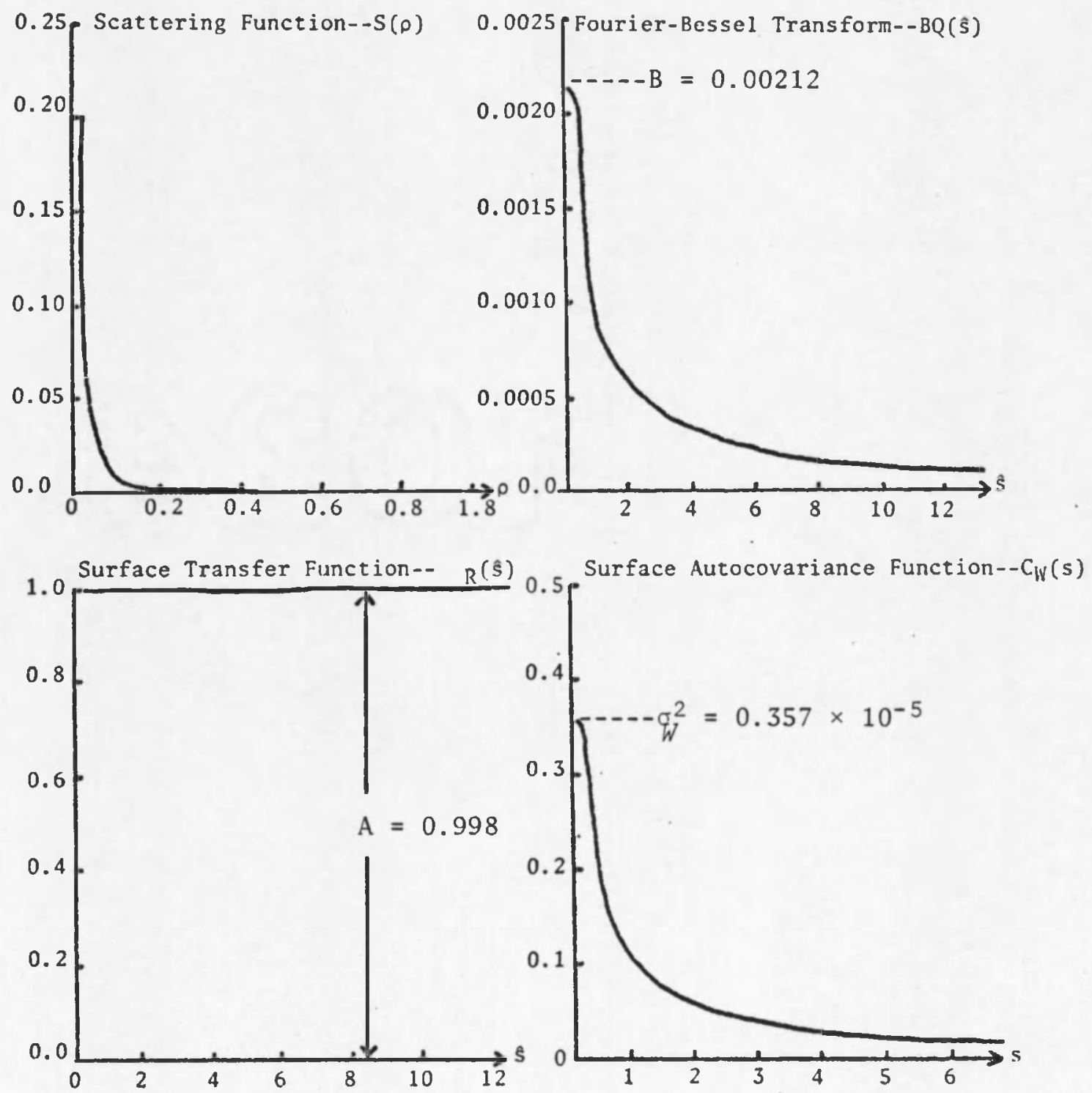

Fig. 5.8. Computer Output from Inverse Scattering Program for Sample \#184 ( $\lambda=0.5145)$. 


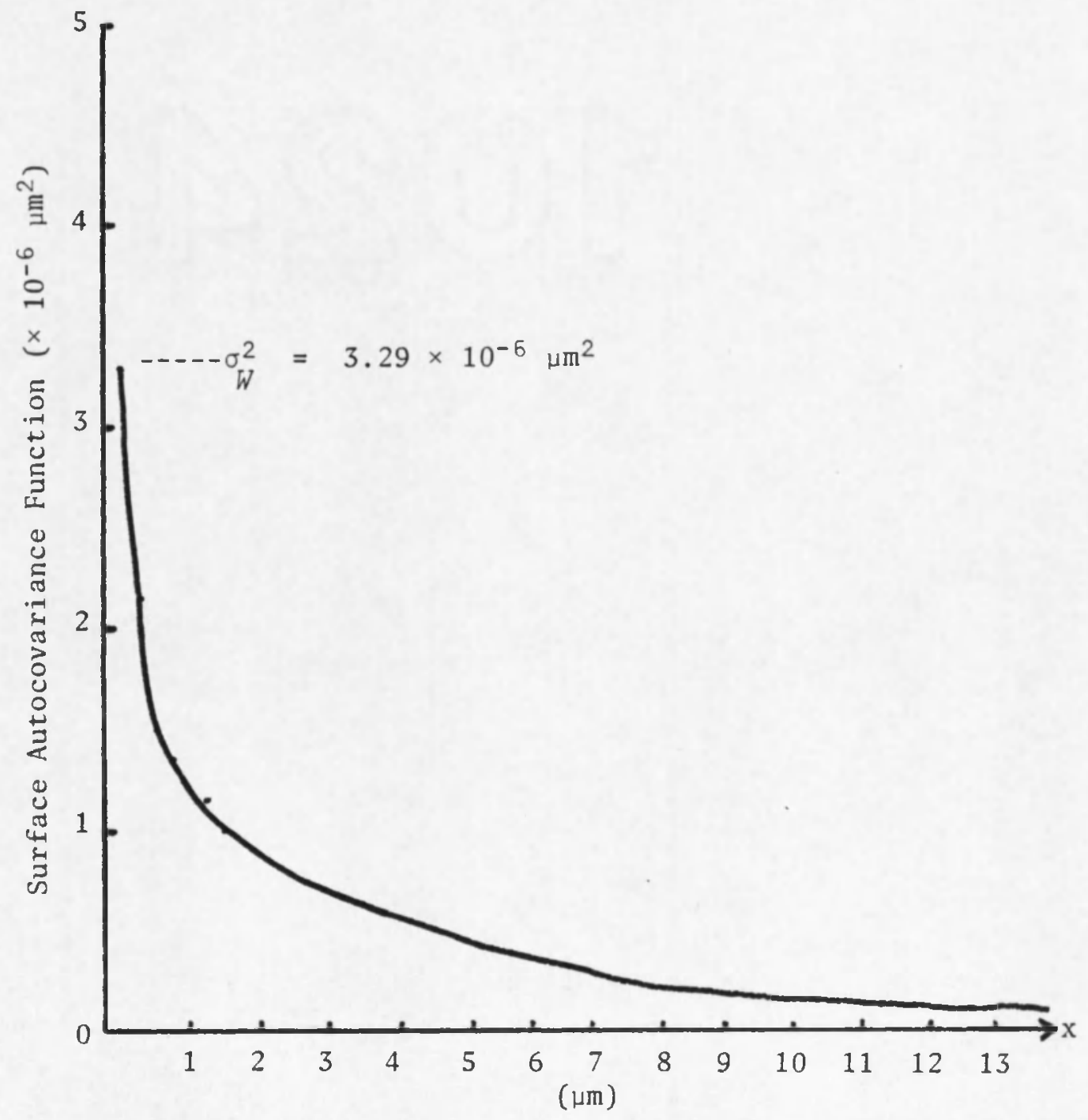

Fig. 5.9. Surface Autocovariance Function for Sample \#198 Predicted from Scattered Light Measurements at $\lambda=0.5145 \mu \mathrm{m}$. 


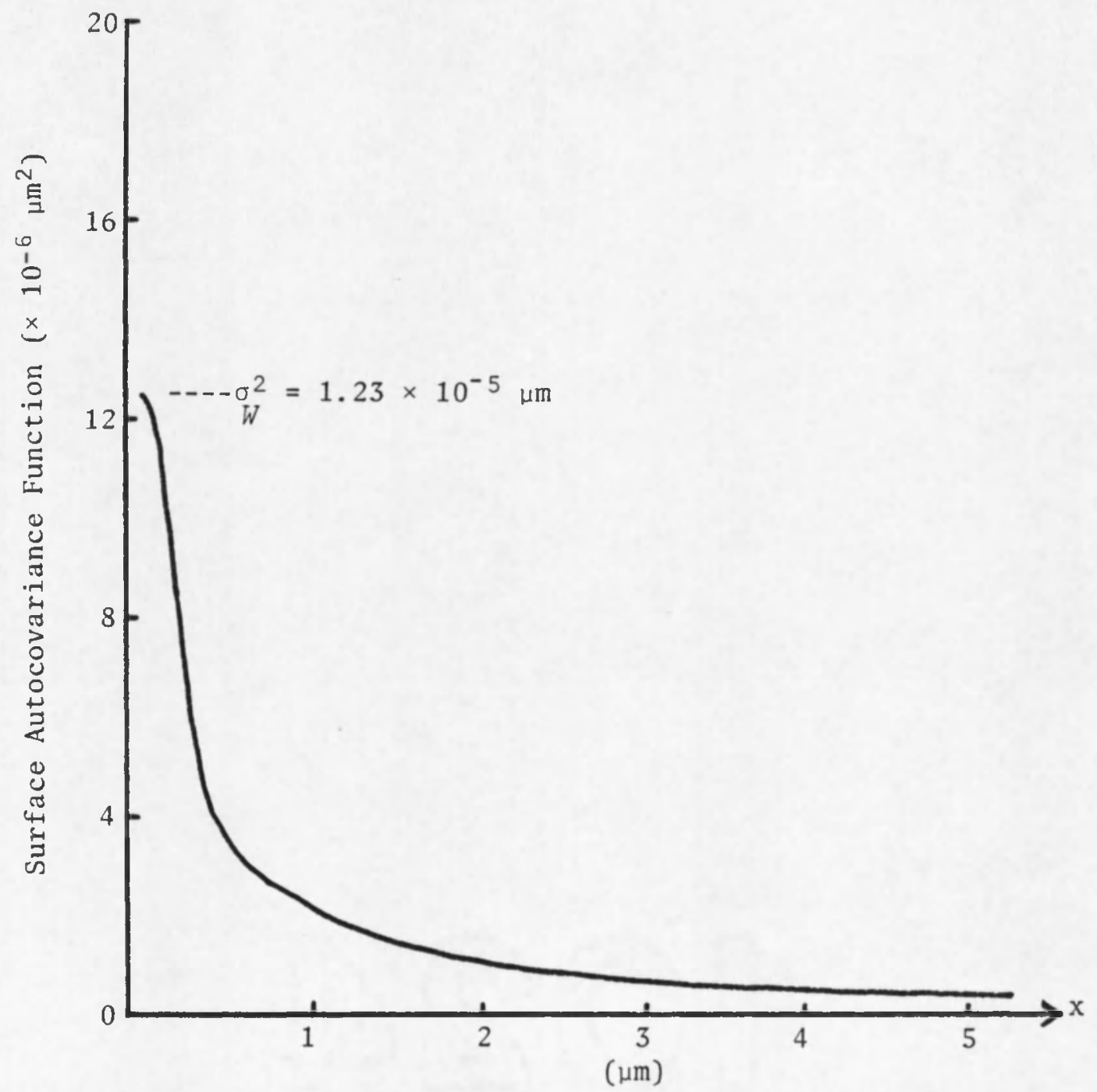

Fig. 5.10. Surface Autocovariance Function for Sample \#186 Predicted from Scattered Light Measurements at $\lambda=0.5145 \mu \mathrm{m}$. 


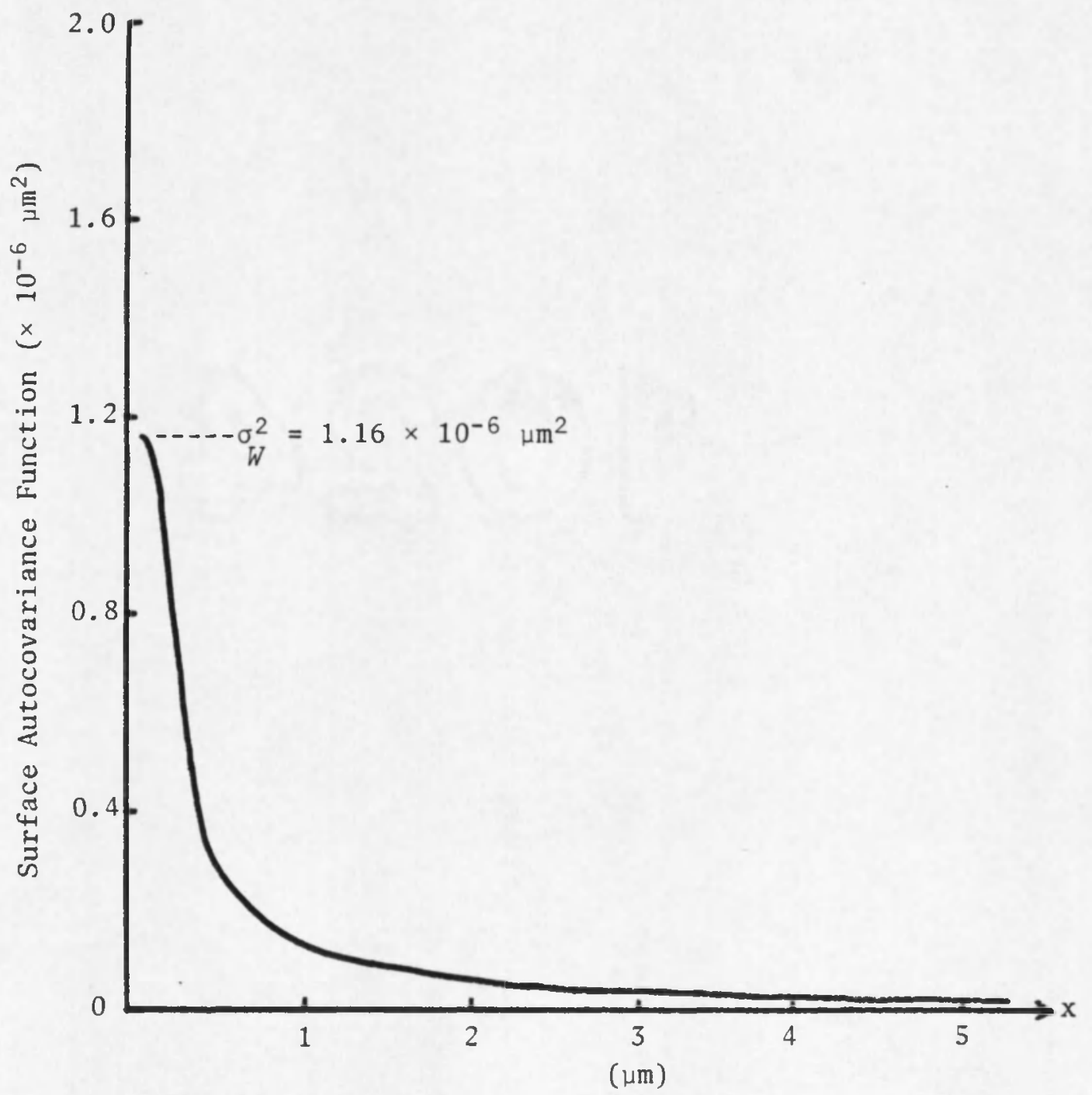

Fig. 5.11. Surface Autocovariance Function for Sample \#163 Predicted from Scattered Light Measurements at $\lambda=0.5145 \mu \mathrm{m}$. 


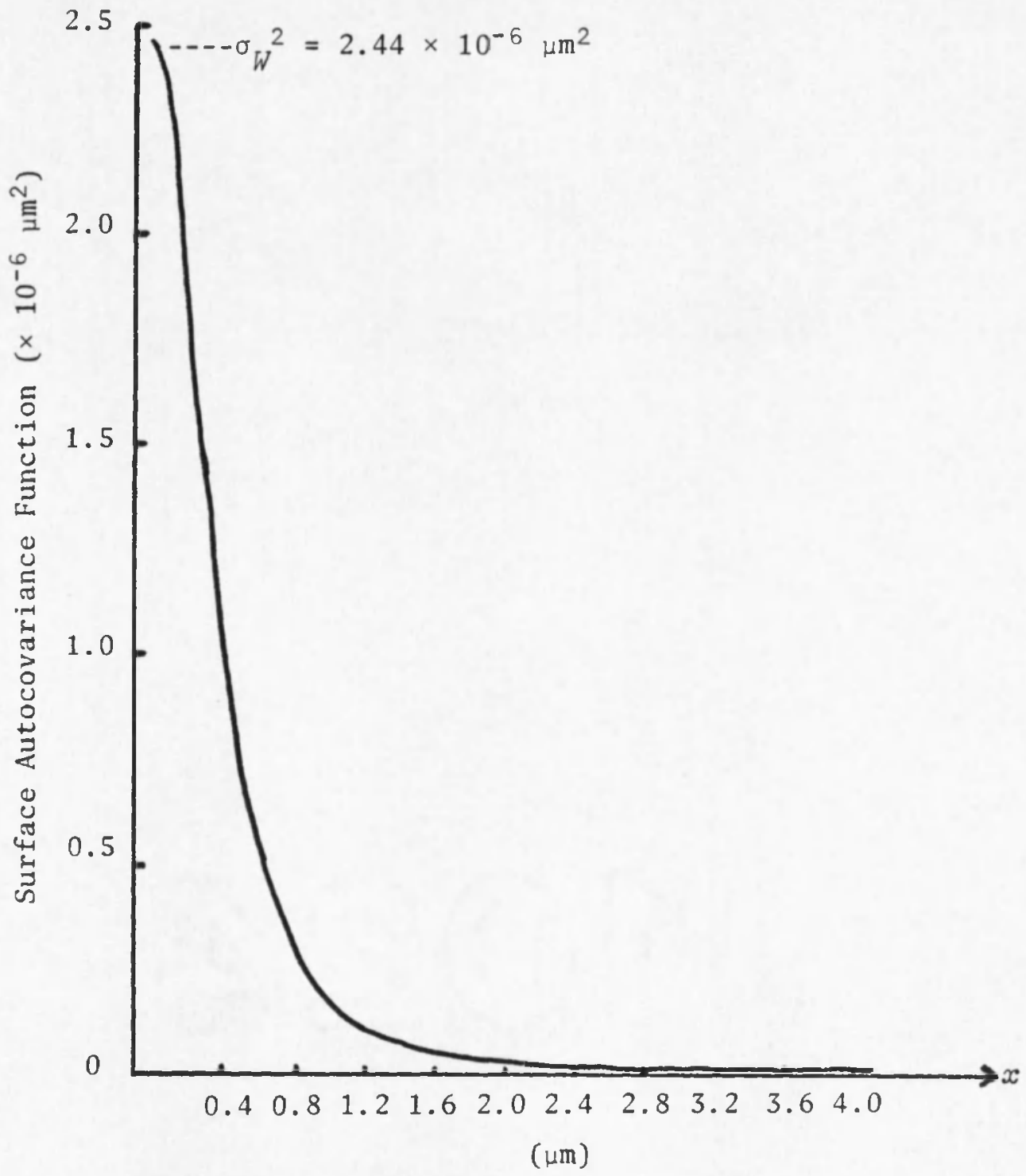

Fig. 5.12. Surface Autocovariance Function for Sample 1NWC Predicted from Scattered Light Measurements at $\lambda=0.5145 \mu \mathrm{m}$. 


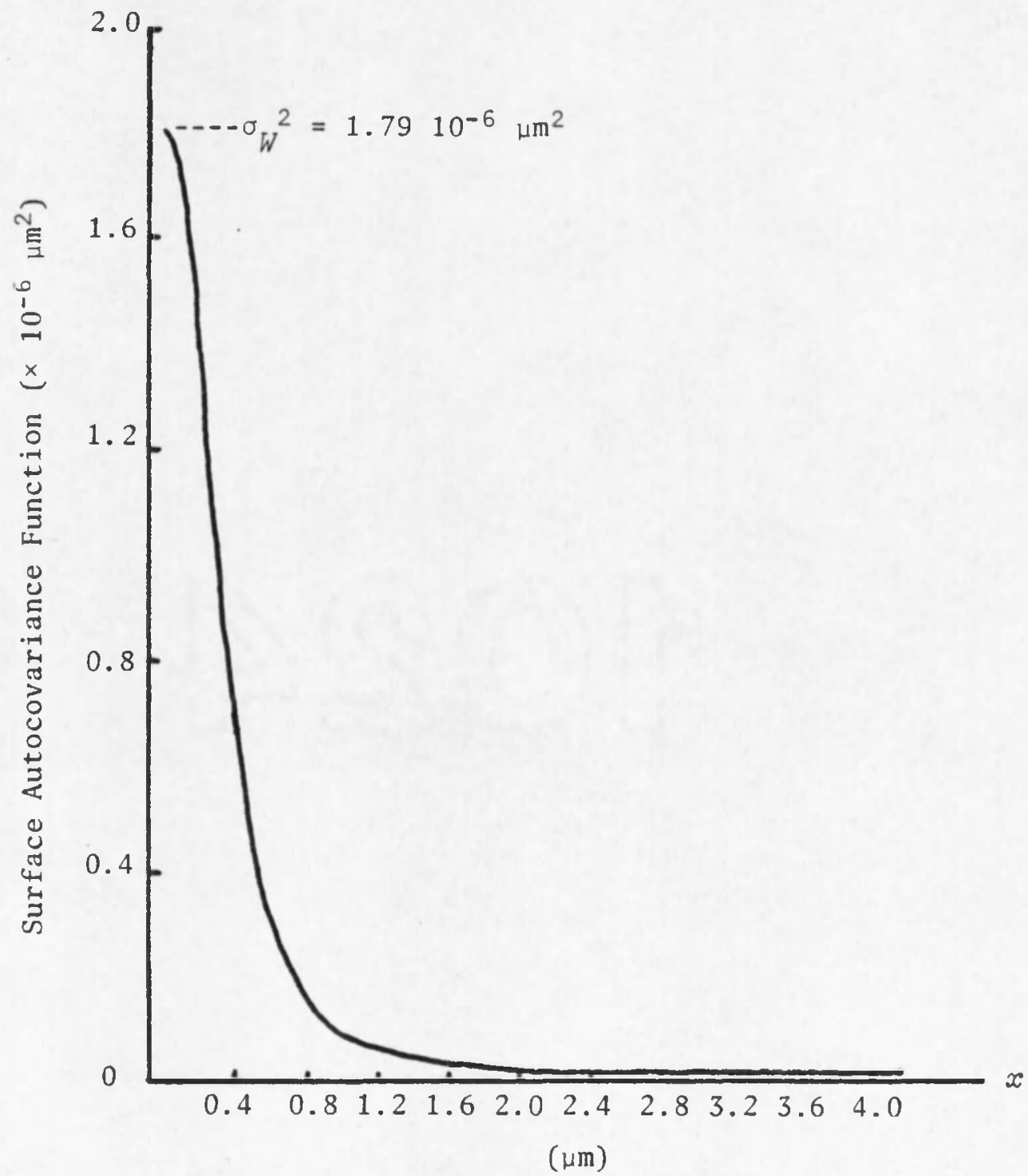

Fig. 5.13. Surface Autocovariance Function for Sample 2NWC Predicted by Scattered Light Measurements at $\lambda=0.5145 \mu \mathrm{m}$. 
Surface Structure Measurements

Three traditional techniques for obtaining surface structure information involve profilometry, electro-microscopy, and FECO interferometry. Most profilometers provide too coarse a measurement for optical surfaces. As illustrated in Fig. 5.2 the electron microscope works nicely on the rough ground glass surfaces but fails to yield sufficient information about the smooth polished surfaces. The FECO interferometer works we11 on smooth surfaces with a strong specular beam but does not yield good results for the rough diffusely reflecting surfaces since it requires multiple reflections. The latter two complementary techniques were thus utilized in our research effort.

Surface profiles of rough samples can be determined from electron-micrograph stereo pairs using conventional stereophotogrammetric techniques (Moffitt, 1959). Nankivel1 (1963) discusses some of the stereo-photogrammetric problems unique to electron microscope applications. Electron-micrograph stereo pairs were produced with both a conventional transmission electron microscope and a scanning electron microscope at a variety of tilt angles and magnifications for several surfaces with known characteristics. Considerable preliminary experimentation with stereo-photogrammetric techniques was then performed with a variety of stereoscope-parallax bar configurations.

A typical electron-micrograph stereo pair is shown in Fig. 5.14 with a line scribed to indicate the position of a set of preliminary surface height measurements that were made with a standard Fairchild Stereocomparagraph. This instrument consists of a mirror stereoscope 

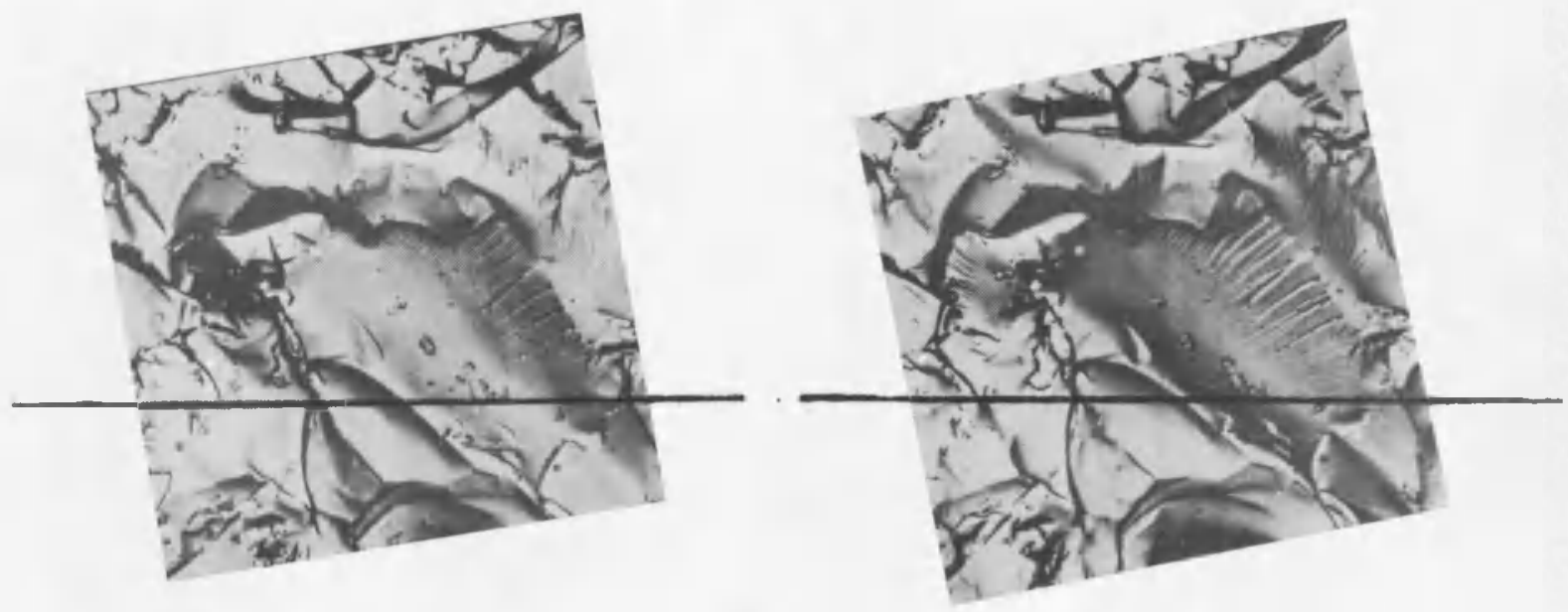



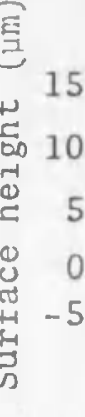

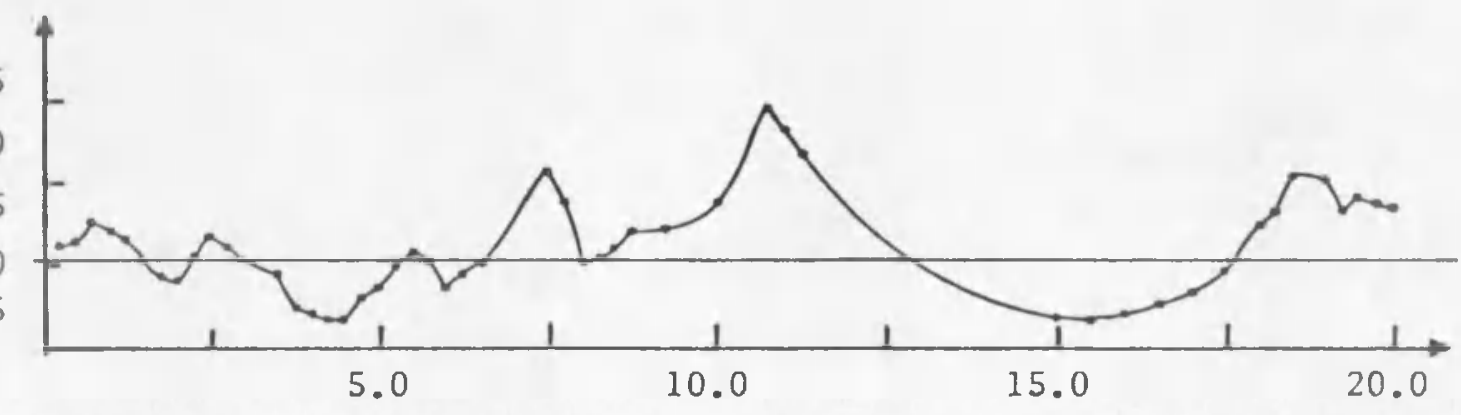

Position along the sample ( $\mu \mathrm{m})$

Fig. 5.14. Typical Electron-Micrograph Stereo Pair of a Ground Glass Sample along with a Surface Profile as Determined by Stereo-Photogrammetric Techniques. 
fitted with a parallax bar containing a micrometer for measuring the parallax of each desired pair of points. The resulting surface profile is also shown in Fig. 5.14. This surface is a very rough ground glass produced with $30 \mu \mathrm{m}$ grit. The tedious procedure of obtaining surface profiles in this manner becomes increasingly more difficult as the surface becomes smoother.

The two statistical parameters which determine the scattered light characteristics are the variance of the surface height distribution and the surface autocorrelation function. A computer program (see Appendix D) was written which takes the surface profile data and determines the above two parameters. An electron micrograph showing the surface of Sample \#172 magnified 10,000 times is illustrated in Fig. 5.15 along with the surface profile, surface height distribution function, and the surface autocovariance function. One hundred-twenty data points were used for obtaining these surface parameters. Additional data was recorded to determine the degree to which the surface was homogeneous and isotropic.

Surface profiles of several smooth samples were measured by Dr. Jean Bennett at the Naval Weapons Center on a scanning FECO interferometer (see Appendix E) capable of determining very small height differences with a lateral resolution of $2 \mu \mathrm{m}$. This instrument, along with auxiliary equipment which includes a slow-scan TV camera, signal averager, minicomputer, and teletype unit, yields the surface profile, rms roughness, surface height distribution function, surface autocovariance function, and other statistical properties of optical surfaces. 
Electron Micrograph

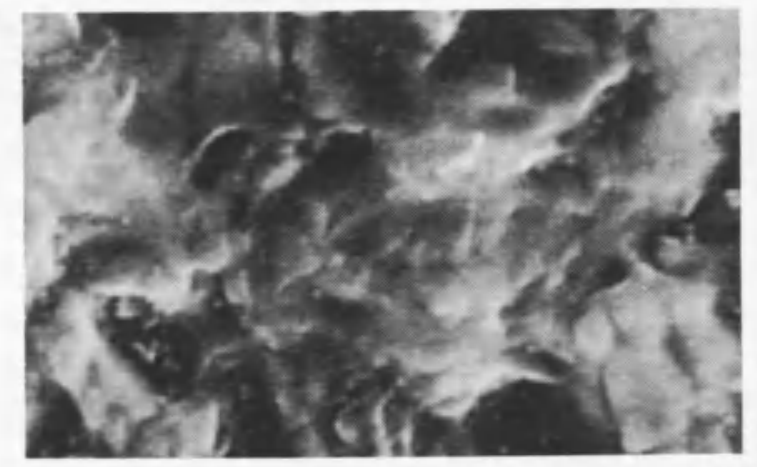

Surface Height

Distribution

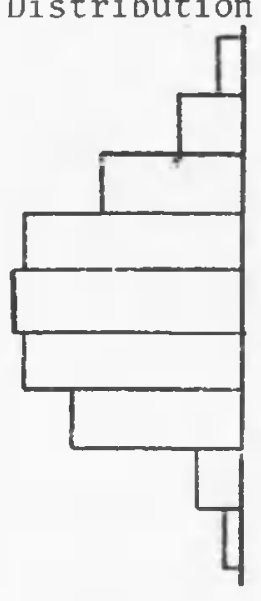

0.5
0.4
0.3
0.2
0.1
-0.1
-0.2
-0.5
-0.4
-0.5

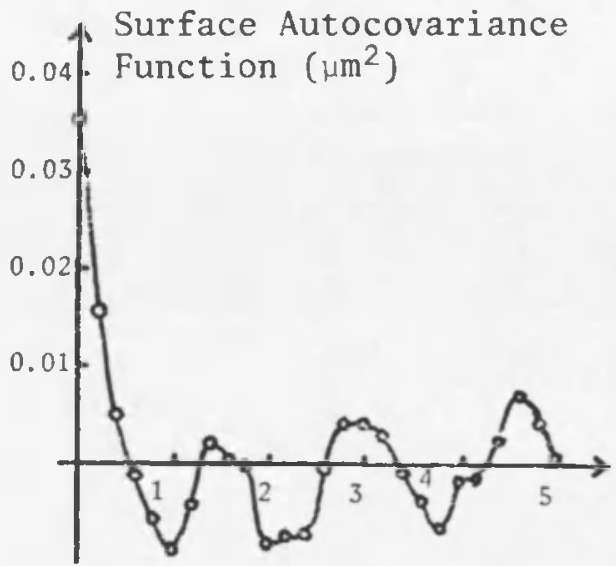

Surface Profile $(\mu \mathrm{m})$

Fig. 5.15. An Electron Micrograph of Sample \#172 is shown along with a Surface Profile, the Surface Height Distribution, and the Surface Autocovariance Function as Determined by Stereo-Photogrammetric Techniques. 
The results of these measurements on Sample \#198 are shown in Fig. 5.16 .

The rms surface roughness was also predicted from visual measurements of the FECO fringe widths, as well as from total integrated scatter measurements performed at the Naval Weapons Center. Additional scattered light measurements made on a few samples allowed the autocovariance length to be calculated when assuming the autocovariance function to be Gaussian.

Comparison of Predictions with Measurements

Theoretical predictions of surface characteristics for Sample \#172 were shown in Fig. 5.7. Direct comparison with the results of the surface measurements shown in Fig. 5.15 indicate that the values for the rms surface roughness differ by approximately a factor of three and the widths of the autocovariance function (distance to the first zero) differ by approximately 20 per cent. Under the circumstances involved this can be considered to be remarkably good agreement since this surface is so rough as to make the theory somewhat suspect and not rough enough for the electron-micrograph stereo measurements to be considered reliable.

The predictions of surface characteristics based upon scattered light measurements from smooth surfaces are tabulated in Table 5.1 along with the results of independent measurements performed at the Naval Weapons Center. The rms surface roughness of a few samples was determined both from total integrated scatter measurements and from 

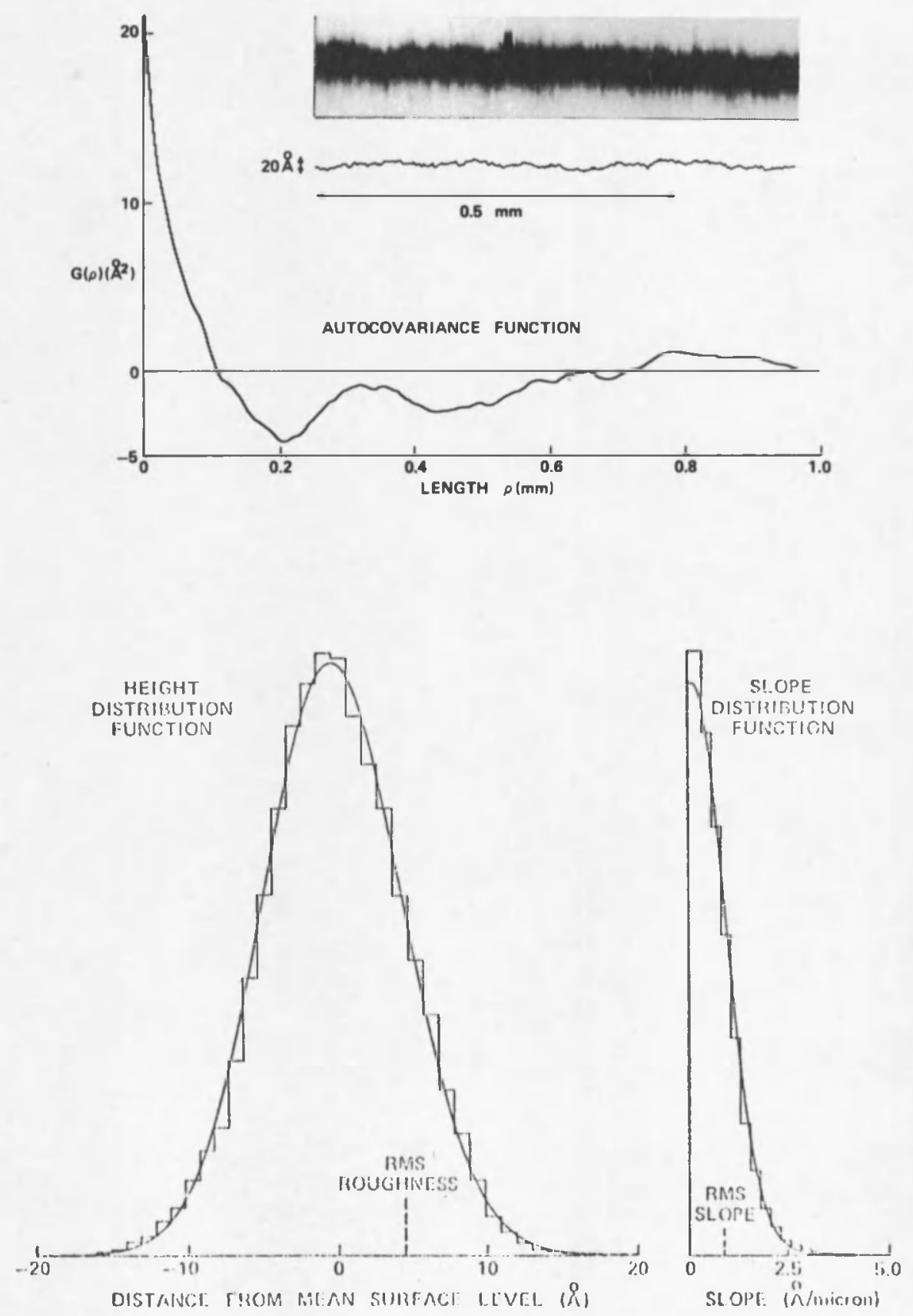

Fig. 5.16. Surface of Sample \#198 as Determined by the Scanning FECO Interferometer at the Naval Weapons Center. 
Table 5.1. Surface Characteristics.

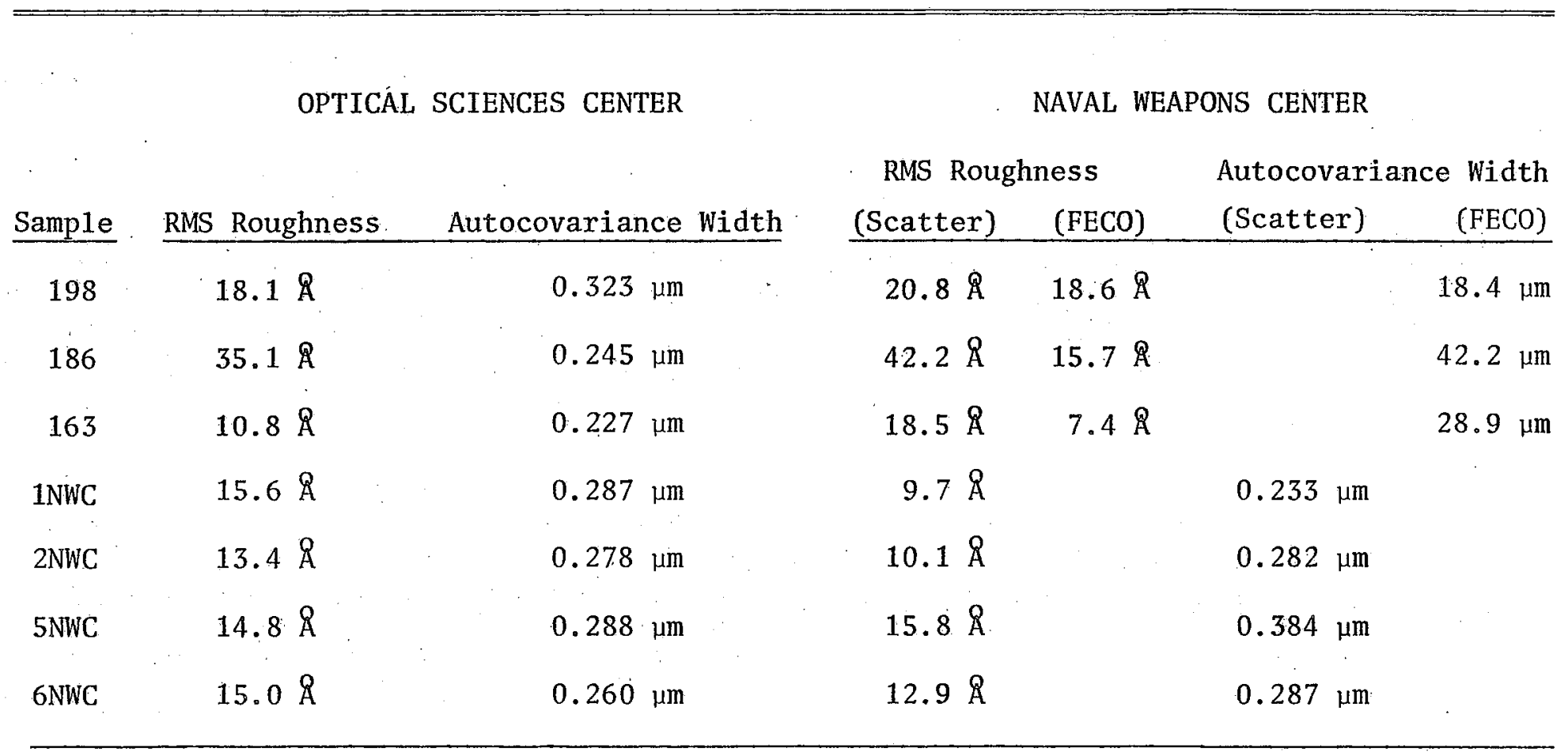


visual measurement of the FECO fringe widths. The wide variation in the results of these two techniques for Sample \#186 and Sample \#163 was attributed to nonuniform roughness over the surface of the sample. The surface autocovariance width determined from the scanning FECO interferometer is one to two orders of magnitude larger than that theoretically predicted from scattered light measurements. This is due to the poor lateral resolution of the instrument. The surface autocovariance width predicted from a simple theory which assumes a Gaussian shape for the autocovariance function does compare favorably with our predictions. The autocovariance widths tabulated in Table 5.1 are arbitrarily chosen to be the half-width of the surface autocovariance function at $1 / \sqrt{\mathrm{e}}$ times its maximum height.

\section{Indirect Verification of Inverse Scattering Solution}

The accurate determination of micro-structure on an optical surface has been shown to be extremely difficult to accomplish by direct measurement (therein lies the motivation for attempting to solve the inverse scattering problem). However, our theoretical treatment of the inverse scattering problem can be tested indirectly by using the surface characteristics predicted from scattered light measurements at a given wavelength to calculate the scattered light behavior at a different wavelength: This calculated scattering function can then be readily compared to the directly measured scattering function at that wavelength. Figure 5.17 provides a direct comparison of the measured scattering function from Sample \#184 at $\lambda=0.6328 \mu \mathrm{m}$ to that predicted from surface characteristics determined from scattered light 


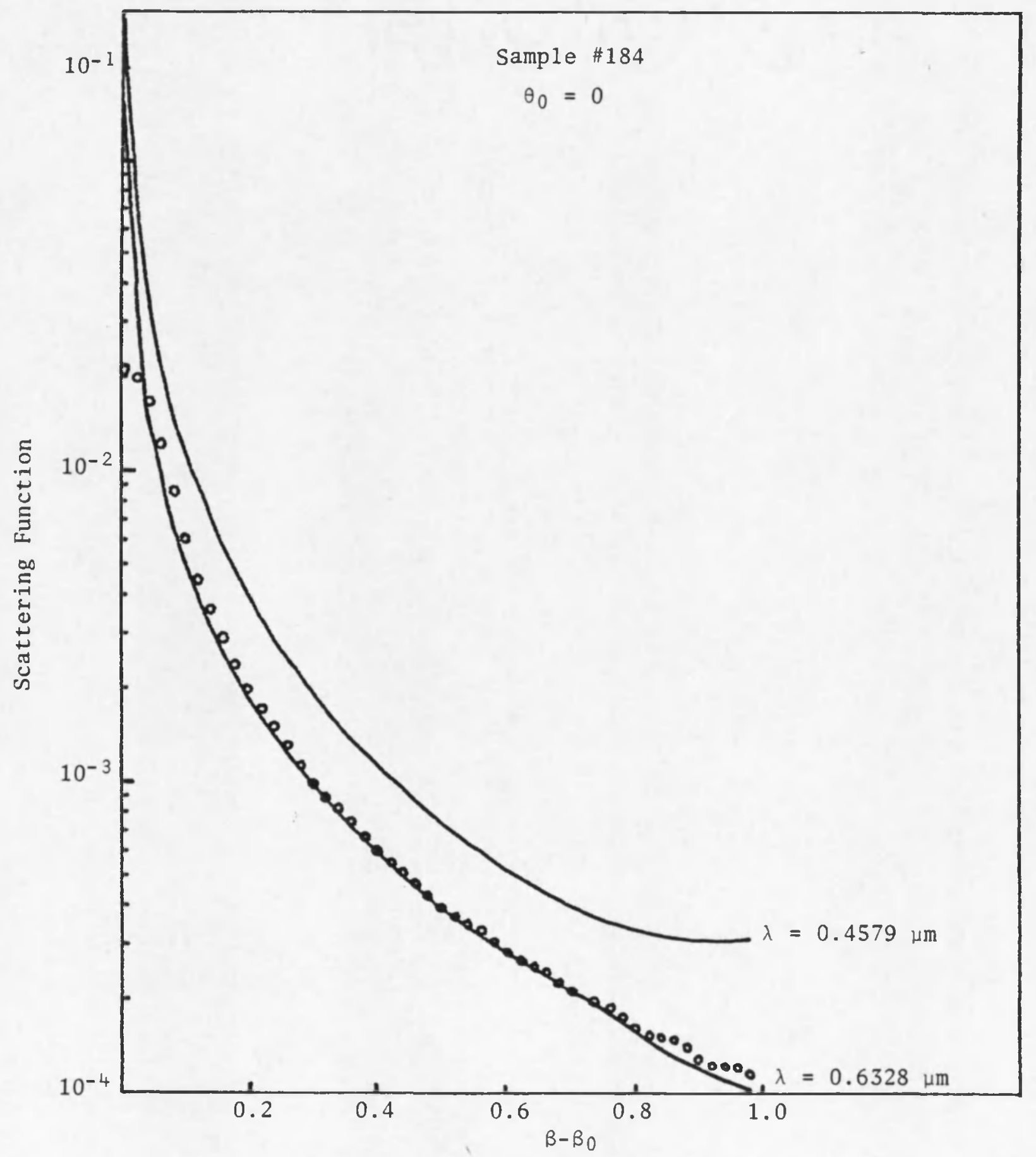

Fig. 5.17. Indirect Verification of Inverse Scattering Problem. 
measurements at $\lambda=0.4579 \mu \mathrm{m}$. The remarkable agreement displayed provides experimental verification of our theoretical treatment of the inverse scattering problem, at least for smooth surfaces.

\section{Wavelength Effects}

The transfer function characterization of scattering surfaces developed in Chapter 3 offers a simple means of determining the wavelength dependence of the scattered light behavior. Once the surface characteristics are known (whether from direct measurement or calculated from scattered light data), the same theory provides a simple method of predicting the scattering function at any desired wavelength.

This technique of predicting the scattering behavior as a function of wavelength has been experimentally verified (see Fig. 5.17) for wavelength ranges limited to the visible spectrum. Furthermore, the same data can be used to verify the wavelength scaling law for smooth surfaces stated in Eq. (70). This is illustrated in Fig. 5.18.

Similar attempts to predict the scattering properties of a surface at a wavelength of $10.6 \mu \mathrm{m}$ from measured data in the visible was not successful. This failure was due to the greatly expanded angular width of the scattering function at long wavelengths as described by the wavelength scaling law, and is illustrated in Fig. 5.19. Note that scattered 1ight measurements over angular range from $1^{\circ}$ to $46^{\circ}$ at a wavelength of $0.4579 \mu \mathrm{m}$ can be used to predict the scattering behavior from $1.4^{\circ}$ to $90^{\circ}$ for a wavelength of $0.6328 \mu \mathrm{m}$. However, these same measurements provide only a few data points in the angular range from $1^{\circ}$ to $2.5^{\circ}$ that are useful in predicting the scattering behavior for a 


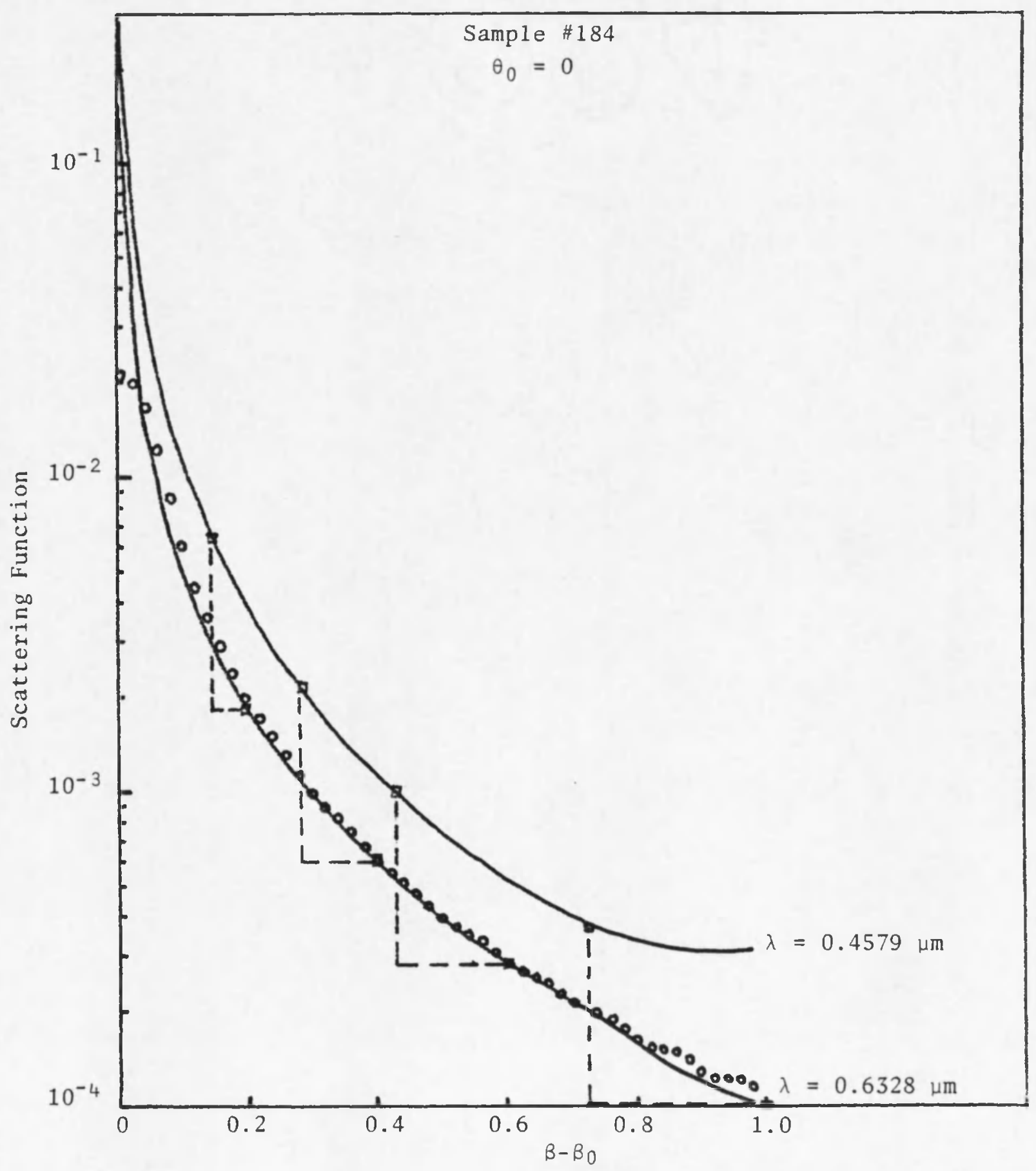

Fig. 5.18. Experimental Verification of Wavelength Scaling Law for Smooth Surfaces. 


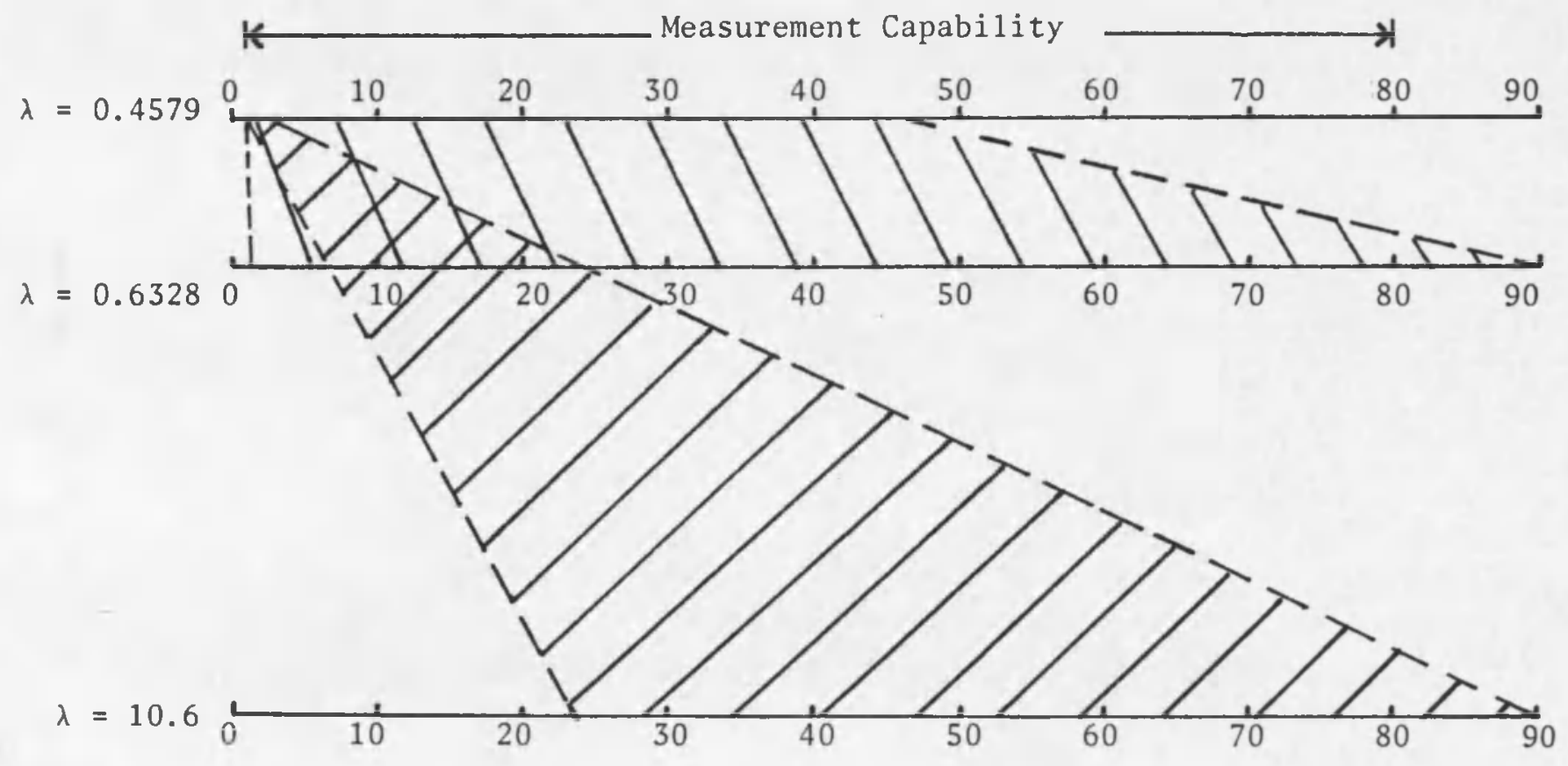

Fig. 5.19. Diagram Illustrating the Effects of the Wavelength Scaling Law. 
wavelength of $10.6 \mu \mathrm{m}$, and no information is obtained concerning the scattered light behavior at angles less than $24^{\circ}$.

It is now clear that it may not be possible to compare the scattered intensity of two widely separated wavelengths in a given direction without extrapolating one curve. However, this behavior has the advantage of allowing one to determine the scattering characteristics at very small angles (unobtainable by direct measurement due to mechanical constraints) by making large angle scatter measurements at a longer wavelength. The angular range of validity for predictions of scattered light behavior based upon measurements from $1^{\circ}$ to $80^{\circ}$ at 10.6 $\mu \mathrm{m}$ is presented as a function of wavelength in Fig. 5.20. For example, measurements from $1^{\circ}$ to $80^{\circ}$ at a wavelength of $10.6 \mu \mathrm{m}$ could be used to predict the scattering properties of visible light $(\lambda=0.5 \mu \mathrm{m})$ in an angular range from approximately $0.045^{\circ}$ to $2.7^{\circ}$. Or conversely, if one has the capability of making very small angle measurements in the visible, the wide angle scattering characteristics at a longer wavelength can be determined. 


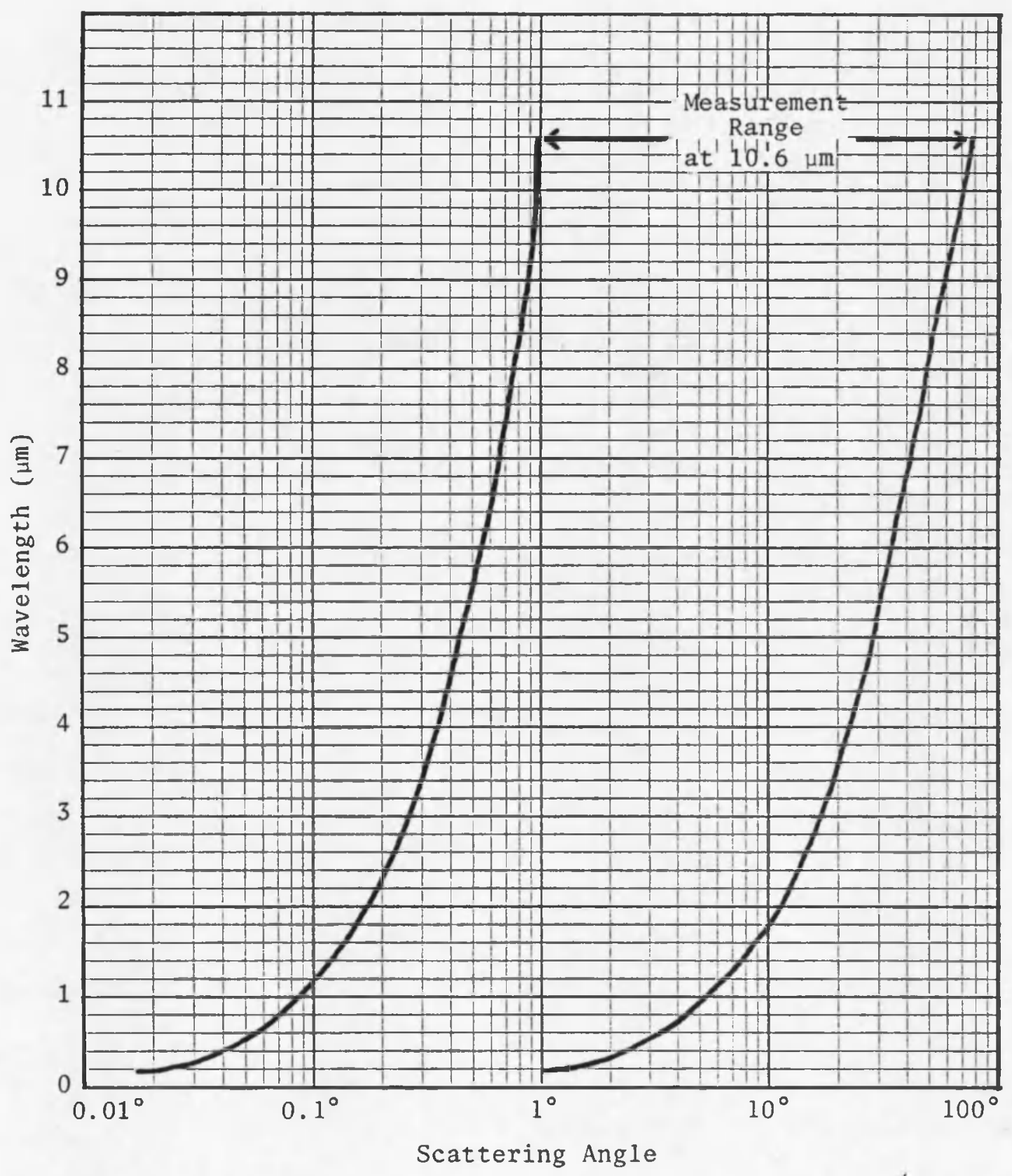

Fig. 5.20. The Angular Range of Validity for Predictions of Scattered Light Behavior Based upon Measurements from $1^{\circ}$ to $80^{\circ}$ at $10.6 \mu \mathrm{m}$. 
CHAPTER 6

\section{CONCLUSION}

This study has been a general investigation of surface scatter phenomena dealing with several different aspects of scattered light behavior. An elementary theoretical development based upon scalar diffraction theory has been presented. Linear systems theory and modern Fourier techniques result in a theoretical model of light scattering systems which closely parallels the highly successful theory of isoplanatic imaging systems. An extensive experimental program has accompanied this theoretical development in an attempt to verify theoretica1 predictions.

\section{Summary of Results}

An analytical expression has been obtained for a surface transfer function which relates the surface micro-structure to the scattered distribution of radiation from that surface. The existence of such a transfer function implies a shift-invariant scattering function which does not change shape with the angle of the incident beam. This is a rather significant development which greatly reduces the quantity of data required to. completely characterize the scattering properties of a surface. For a large class of well-behaved surfaces this transfer function is described in terms of only the rms surface roughness and the surface autocovariance function. This transfer function thus 
provides a straightforward solution to the inverse scattering problem (i.e., determining the surface characteristics from scattered light measurements). Once the surface characteristics are known, the same theory provides an equally simple method of predicting the wavelength dependence of the scattered light distribution.

Experimenta1 verification of the shift-invariant scattering function has been successfully demonstrated for smooth surfaces $\left(\sigma_{W}<<\right)$. The scattered light measurements from rough (diffusely reflecting) surfaces results in a scattering function which is shift-invariant over only a smal1 range of angles and departs significantly from the predicted behavior at large scattering angles.

A computer program has been developed that operates upon scattered light data to yield the total integrated scatter, the surface transfer function; the rms surface roughness, and the surface autocovariance function. Although accurate determination of micro-structure on optical surfaces is extremely difficult to accomplish by direct measurement (thus the motivation for attempting to solve the inverse scattering problem), favorable comparisons of predicted surface characteristics with the corresponding measured quantities have been demonstrated for both smooth surfaces and moderately rough surfaces. In addition, experimental verification of the inverse scattering program was accomplished indirectly by supplying scattered light data of one wavelength as input to the inverse scattering program in order to determine the relevant surface characteristics; then this information was used to predict the scattering function at a different wavelength. 
Excellent agreement with the measured scattering function at that wavelength was achieved.

Since the above technique involves numerical computations on sampled data, an analytical expression for a wavelength scaling law is not required to determine the scattering function at any desired wavelength. However, in order to gain insight into the wavelength dependence of surface scatter phenomena, a wavelength scaling law for smooth surfaces was derived and verified. This scaling law consists of a change in the scattering angle as well as a change in the amplitude of the scattering function with changes in wavelength. It therefore provides a valuable tool for predicting the scattering behavior in certain angular regions or wavelength ranges where direct measurements are difficult to obtain.

\section{Further Theoretical Considerations}

No explicit approximations concerning the size of the surface variations were made in the theoretical development presented in Chapter 3. However, a simplifying assumption was made regarding the random component of the pupil function described in Eq. (33). It was assumed that the phase variations in the disturbance emerging from the scattering surface were equal to the perturbations introduced onto a normally incident wavefront. Careful examination of Fig. 6.1 reveals that the phase difference introduced by reflection from a rough surface depends upon both the angle of incidence and the angle of observation in addition to the surface height at the point of reflection. The phase variations along the scattering surface can thus be expressed as 


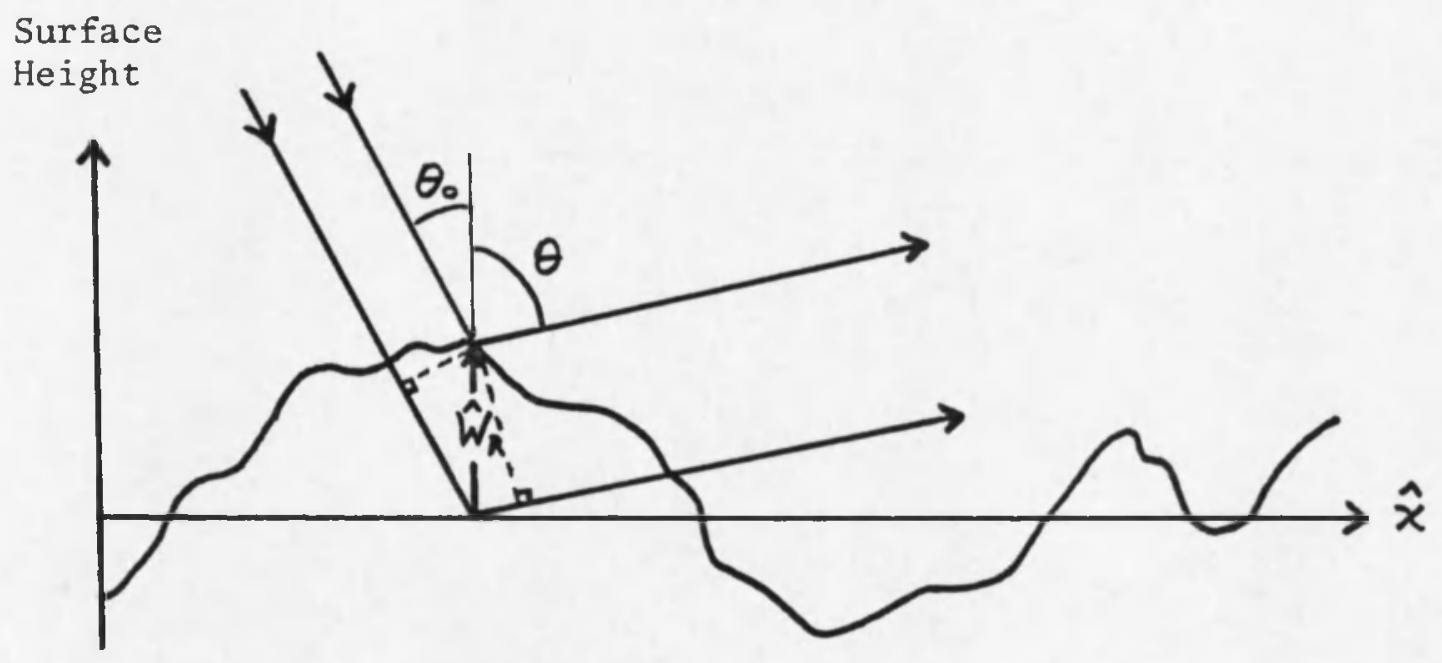

Fig. 6.1. Illustration of the Phase Variation Introduced by Reflection from a Rough Surface. 


$$
\phi(\hat{x}, \hat{y})=2 \pi\left(\gamma+\gamma_{0}\right) \hat{W}_{R}(\hat{x}, \hat{y})
$$

where

$$
\gamma=\cos \theta, \gamma_{0}=\cos \theta_{0} .
$$

The effective transfer function of a scattering surface is now given by the following general expression

$$
\mathscr{H}_{R}(\hat{x}, \hat{y})=e^{-\left[2 \pi\left(\gamma+\gamma_{0}\right) \sigma_{\hat{W}}{ }^{2}\right]}\left[1-\frac{C W(\hat{x}, \hat{y})}{\sigma_{\hat{W}}^{2}}\right]
$$

This expression can be interpreted as a two-parameter family of transfer functions, one for every possible angle of incidence and every possible scattering angle.

This generalization still leaves us with a theoretical model closely paralleling that of non-isoplanatic imaging systems which can be characterized by a different transfer function for each offaxis object point. It therefore seems reasonable that an "aberration theory" of scattering systems can be developed to provide more insight into the scattering behavior of rough surfaces.

A preliminary empirical search for a new scattering function of the form $\left(\gamma+\gamma_{0}\right)^{m} \beta(\alpha, \beta)$ plotted versus the quantity $\left(\beta-\beta_{0}\right) /\left(\gamma+\gamma_{0}\right)^{n}$ resulted in the following interesting dilemma. When $m=2$ and $n=1$, the scattering function for Sample \#172 indeed becomes quite shift-invariant with respect to changes in the incident angle (there is a theoretical basis for this particular form of the scattering function if some assumptions are made concerning the shape of the surface autocovariance function). However, this same scattering function degrades the 
shift-invariance of Sample \#200. Clearly a more general theory should also work for the special case of smooth surfaces.

\section{Suggestions for Further Research}

The following suggestions are made for further research in the area of surface scatter phenomena:

1. Continue the theoretical development on the transfer function characterization of scattering surfaces. This should include an attempt to solve the inverse scattering problem for rough surfaces by using the general expression for the transfer function described by Eq. (79). Empirical curve fitting techniques may be useful in an attempt to discover new scattering functions or plotting techniques which result in shift-invariant behavior with respect to changes in the incident angle. Generalizing the surface scatter theory to a complete vector treatment while maintaining the transfer function approach would be a major contribution to the understanding of surface scatter phenomena.

2. Improve the scattered light measuring capabilities by obtaining laser sources that span a larger wavelength range.

3. Automate the scattering apparatus for high-speed acquisition and analysis.

4. Acquire samples and perform measurements upon selected moderately rough surfaces $(0.1<\hat{\sigma}<1.0)$ and compare with theoretical predictions. 


\section{APPENDIX A}

\section{CALCULATION OF ABERRATION COEFFICIENTS FOR DIFFRACTED WAVE FIELDS}

For the case of a plane wave incident upon an aperture, the diffracted wave field on an observation plane is given by Eqs. (16) and (17), where

$$
\hat{W}=(\hat{l}-\hat{z})+\left(\hat{x}^{\prime} \hat{x}+\hat{y}^{\prime} \hat{y}\right) / \hat{z} \text {. }
$$

The quantity $\hat{l}$ can be written as

$$
\begin{aligned}
\hat{l} & =\sqrt{\left(\hat{x}-\hat{x}^{\prime}\right)^{2}+\left(\hat{y}-\hat{y}^{\prime}\right)^{2}+\hat{z}^{2}} \\
& =\hat{z} \sqrt{1+\left[\hat{s}^{2}+\hat{s}^{\prime 2}-2\left(\hat{x} \hat{x}^{\prime}+\hat{y} \hat{y}^{\prime}\right)\right] / \hat{z}^{2}}
\end{aligned}
$$

where

$$
\hat{s}^{2}=\hat{x}^{2}+\hat{y}^{2}, \quad \hat{s}^{\prime 2}=\hat{x}^{\prime 2}+\hat{y}^{\prime 2} \text {. }
$$

A binomial expansion of the above square root results in the following expression for $\hat{W}$

$$
\begin{aligned}
\hat{W} & =\frac{\hat{z}}{2}\left[\hat{s}^{2}+\hat{s}^{\prime 2}\right] / \hat{z}^{2} \\
& -\frac{\hat{z}}{8}\left[\hat{s}^{4}+\hat{s}^{\prime 4}+4\left(\hat{x} \hat{x}^{\prime}+\hat{y} \hat{y}^{\prime}\right)^{2}+2 \hat{s}^{2} \hat{s}^{\prime 2}-4 \hat{s}^{2}\left(\hat{x} \hat{x}^{\prime}+\hat{y} \hat{y}^{\prime}\right)-4 s^{\prime 2}\left(x x^{\prime}+y y^{\prime}\right)\right] / z^{4} \\
& + \text { higher-order terms. }
\end{aligned}
$$


If we assume a rotationally-symmetric diffracting aperture we can, without loss of generality, choose the observation point on the $\hat{y}$-axis. Let us therefore set $\hat{x}=0$. We can then let $\hat{s}=\hat{y}$ and $\hat{y}^{\prime}=\hat{s}^{\prime} \cos \phi$, hence

$$
\begin{aligned}
\hat{W}= & \frac{\hat{z}}{2}\left[\hat{y}^{2}+\hat{s}^{\prime 2}\right] / \hat{z}^{2} \\
- & \frac{\hat{z}}{8}\left[\hat{y}^{4}+\hat{s}^{\prime 4}+4 \hat{y}^{2} \hat{s}^{\prime 2} \cos ^{2} \phi+2 \hat{y}^{2} \hat{s}^{\prime 2}-4 \hat{y}^{3} \hat{s}^{\prime} \cos \phi\right. \\
& \left.-4 \hat{y}^{\prime 3} \cos \phi\right] / \hat{z}^{4}
\end{aligned}
$$

+ higher-order terms.

If we now substitute

$$
\hat{y}=\rho \hat{y}_{\max }, \quad \hat{s}^{\prime}=\hat{a} \frac{\hat{d}}{2}
$$

into the previous equation, we obtain

$$
\begin{aligned}
& \hat{W}=\frac{\hat{z}}{2}\left[\hat{y}_{\max }{ }^{2} \rho^{2}+(\hat{d} / 2)^{2} \hat{a}^{2}\right] / \hat{z}^{2} \\
& -\frac{\hat{z}}{8}\left[\hat{y}_{\max }{ }^{4} \rho^{4}+(\hat{d} / 2)^{4} \hat{a}^{4}+4 \hat{y}_{\max }{ }^{2}(\hat{d} / 2)^{2} \rho^{2} \hat{a}^{2} \cos ^{2} \phi\right. \\
& +2 \hat{y}_{\max }{ }^{2}(\hat{d} / 2)^{2} \rho^{2} \hat{a}^{2}-4 \hat{y}_{\max }{ }^{3}(\hat{d} / 2) \rho^{3} \hat{a} \cos \phi \\
& \left.-4 \hat{y}_{\max }^{3}(\hat{d} / 2)^{3} \rho \hat{a}^{3} \cos \phi\right] / \hat{z}^{4}
\end{aligned}
$$$$
+ \text { higher-order terms. }
$$

Equating coefficients of corresponding terms between this equation and the wavefront aberration function given by Eq. (24), we obtain the aberration coefficients tabulated in the first column of Table 2.1. 
If we now have a spherical wave incident upon the aperture, the quantity $\hat{W}$ in Eq. (17) is given by

$$
\hat{W}=(\hat{\ell}-\hat{z})-\left(\hat{\ell}_{0}-\hat{z}\right)+\left(\hat{x} \hat{x}^{\prime}+\hat{y} \hat{y}^{\prime}\right) / \hat{z},
$$

where

$$
\hat{l}_{0}=\sqrt{\hat{x}^{12}+\hat{y}^{12}+\hat{z}^{2}}=\hat{z} \sqrt{1+\hat{s}^{12 / \hat{z}^{2}}} \text {. }
$$

A binomial expansion of this quantity results in

$$
\hat{l}_{0}-\hat{z}=\frac{\hat{z}}{2}\left(\hat{s}^{\prime} / \hat{z}\right)^{2}-\frac{\hat{z}}{8}\left(\hat{s}^{\prime} / \hat{z}\right)^{4}+\ldots
$$

But these will merely cancel identical terms in the previous expansion thus resulting in the aberration coefficients presented in column two of Table 2.1.

For the case of a hemispherical observation space the diffracted wave field is given by Eqs. (22) and (23). With a plane wave incident upon the aperture, we have

$$
\hat{W}=(\hat{\ell}-\hat{x})+\left(\alpha \hat{x}^{\prime}+\beta \hat{y}^{\prime}\right) \text {. }
$$

The quantity $\hat{l}$ can be written as

$$
\begin{aligned}
l & =\sqrt{\left(\hat{x}-\hat{x}^{\prime}\right)^{2}+\left(\hat{y}-\hat{y}^{\prime}\right)^{2}+\hat{z}^{2}} \\
& =\hat{r} \sqrt{1+\left[\hat{s}^{\prime 2}-2 \hat{r}\left(\alpha \hat{x}^{\prime}+\beta \hat{y}^{\prime}\right)\right] / \hat{r}^{2}},
\end{aligned}
$$

where

$$
\hat{s}^{\prime 2}=\hat{x}^{\prime 2}+\hat{y}^{\prime 2}, \quad \hat{r}^{2}=\hat{x}^{2}+\hat{y}^{2}+\hat{z}^{2} \text {. }
$$


A binomial expansion of the above square root yields

$$
\begin{aligned}
\hat{W}= & \frac{\hat{r}}{2}\left(\hat{s}^{\prime} / \hat{x}\right)^{2}-\frac{\hat{r}}{8}\left[\hat{s}^{\prime 4}-4 \hat{r} \hat{s}^{\prime 2}\left(\alpha \hat{x}^{\prime}+\beta \hat{y}^{\prime}\right)+4 \hat{r}^{2}\left(\alpha \hat{x}^{\prime}+\beta \hat{y}^{\prime}\right)^{2}\right] / \hat{r}^{4} \\
& + \text { higher-order terms. }
\end{aligned}
$$

If we again assume a rotationally-symmetric diffracting aperture we can, without loss of generality, choose the observation point on the $\hat{y}$-axis. Let us therefore set $\alpha=0$. We can also let $\hat{y}^{\prime}=\hat{s}^{\prime} \cos \phi$ which results in

$$
\begin{aligned}
\hat{W}= & \frac{\hat{r}}{2}\left(\hat{s}^{\prime} / \hat{r}\right)^{2}-\frac{\hat{r}}{8}\left[\hat{s}^{\prime 4}-4 \hat{r} \beta \hat{s}^{\prime 3} \cos \phi+4 \hat{r}^{2} \beta^{2} \hat{s}^{\prime 2} \cos ^{2} \phi\right] / \hat{r}^{4} \\
& + \text { higher-order terms. }
\end{aligned}
$$

If we now substitute

$$
\beta=\rho B_{\max }, \quad \hat{s}^{\prime}=\hat{a} \frac{\hat{d}}{2}
$$

into the previous equation, we obtain

$$
\begin{aligned}
& \hat{W}=\frac{\hat{r}}{2}(\hat{d} / 2 \hat{r})^{2} \hat{a}^{2}-\frac{\hat{r}}{8}\left[(\hat{d} / 2)^{4} \hat{a}^{4}-4 \hat{r} \beta_{\max }(\hat{d} / 2)^{3} \beta \rho^{3} \cos \phi\right. \\
& \qquad+4 \hat{r}^{2} \beta_{\left.\max ^{2}(\hat{d} / 2)^{2} \beta^{2} \rho^{2} \cos ^{2} \phi\right] / \hat{r}^{4}} \\
& + \text { higher-order terms. }
\end{aligned}
$$

Again equating coefficients of corresponding terms between this equation and the wavefront aberration function given by Eq. (24), we obtain the aberration coefficients tabulated in column three of Table 2.1. 
If we now have a spherical wave illuminating the aperture and a hemispherical observation space, the quantity $\hat{W}$ in Eq. (23) is given by

$$
\hat{W}=(\hat{l}-\hat{r})-\left(\hat{l}_{0}-\hat{r}\right)+\left(\alpha \hat{x}^{\prime}+\beta \hat{y}^{\prime}\right) \text {, }
$$

where

$$
\hat{l}_{0}=\sqrt{\hat{x}^{12}+\hat{y}^{12}+\hat{r}^{2}}=\hat{p} \sqrt{1+\hat{s}^{12 / \hat{r}^{2}}} \text {. }
$$

A binomial expansion of this quantity results in

$$
\hat{l}_{0}-\hat{r}=\frac{\hat{r}}{2}\left(\hat{s}^{\prime} / \hat{r}^{2}\right)^{2}-\frac{\hat{r}}{8}\left(\hat{s}^{\prime} / \hat{r}\right)^{4}+\ldots .
$$

Once again these terms merely cancel identical terms in the previous expansion, leaving only coma and astigmatism present in the diffracted wave field as indicated in the last column of Table 2.1. 


\section{APPENDIX B}

\section{BIDIRECTIONAL REFLECTANCE DISTRIBUTION FUNCTION}

The basic quantity that characterizes (geometrically) the reflecting properties of a surface element $d A$ is the bidirectional reflectance distribution function (BRDF). This quantity

$$
\begin{aligned}
f_{r}\left(\theta_{i}, \phi_{i} ; \theta_{r}, \phi_{r}\right) & =d L_{r}\left(\theta_{i}, \phi_{i} ; \theta_{r}, \phi_{r} ; E_{i}\right) / d E_{i}\left(\theta_{i}, \phi_{i}\right) \\
& =d L_{r}\left(\theta_{i}, \phi_{i} ; \theta_{r}, \phi_{r} ; E_{i}\right) / L_{i}\left(\theta_{i}, \phi_{i}\right) d \Omega_{i}\left(\mathrm{sr}^{-1}\right)
\end{aligned}
$$

is defined by Nicodemus (1970), as the reflected radiance

$d L_{r}\left(\theta_{i}, \phi_{i} ; \theta_{r}, \phi_{r} ; E_{i}\right)$ of the surface element $d A$ in the direction $\left(\theta_{r}, \phi_{r}\right)$ divided by the incident irradiance $d E_{i}\left(\theta_{i}, \phi_{i}\right)=L_{i}\left(\theta_{i}, \phi_{i}\right) d \Omega_{i}$ producing it. The geometry of this situation is illustrated in Fig. B.1, where the element of projected solid angle is given by $d \Omega \equiv \cos \theta d \omega$.

The numerical value of the BRDF for a given pair of incident and reflected ray directions may vary from zero to infinity. In particular, consider two ideal cases. The BRDF is a constant for all reflected directions for a perfectly diffuse (Lambertian) surface; and it becomes infinite (as a Dirac delta function) for a perfectly specular reflector. The BRDF, defined above as a ratio of infinitesimals, is an idealized concept that can never be measured exactly. Real measurements are 


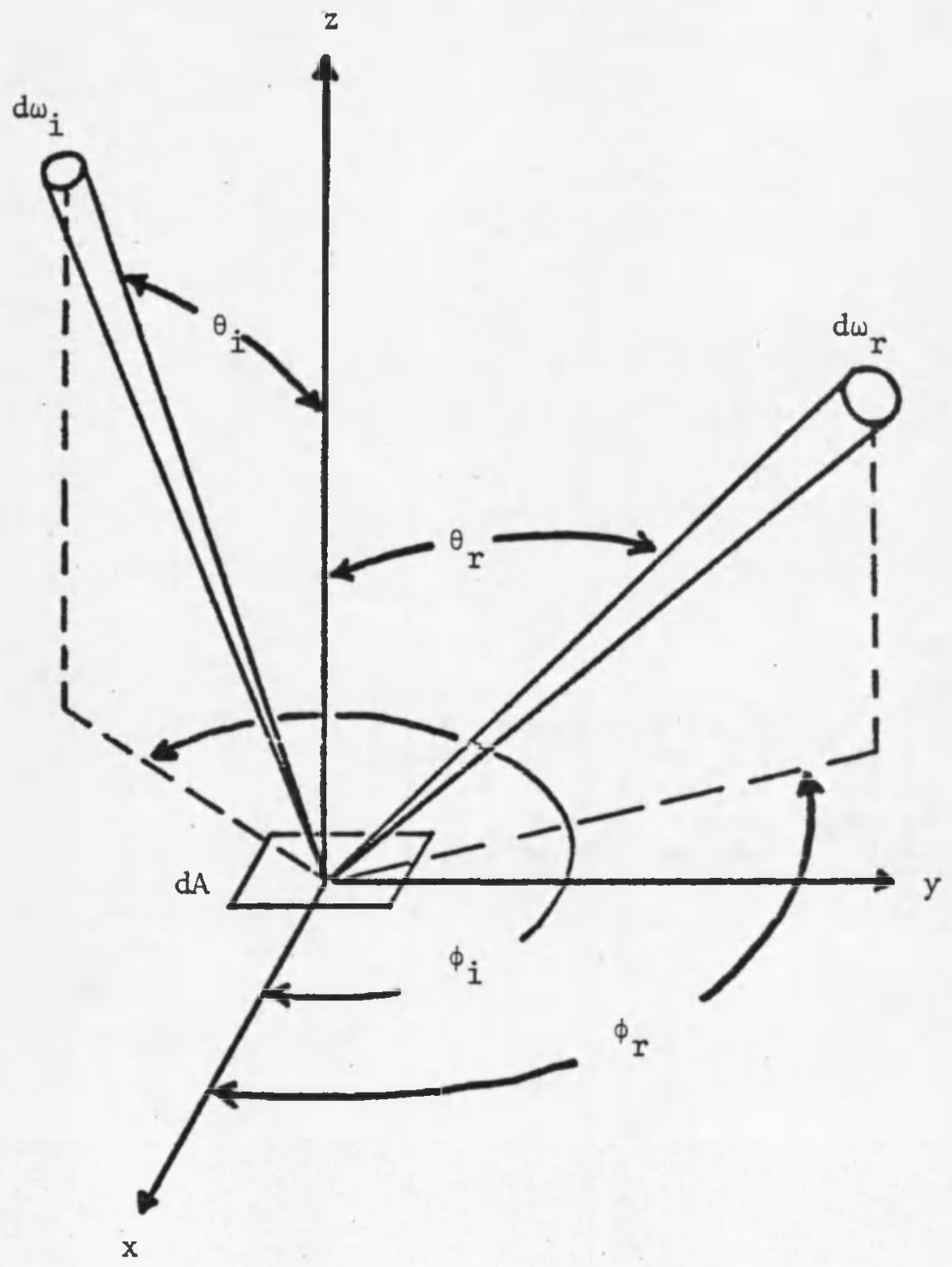

Fig. B.1. Geometry of Incident and Reflected Elementary Beams Used to Define the Bidirectional Reflectance Distribution Function. 
always made over some finite solid angle and wavelength interval and can therefore yield only average values $\overline{f_{p}}$ over those parameter intervals.

The BRDF is basic in the sense that all other reflectance or scattering functions can be derived from it. For example, Judd (1967) lists nine different kinds of reflectance functions based on the angular extent of the incident and reflected radiation. All of them can be derived from the BRDF.

Note that the BRDF is a four-dimensional quantity that can be thought of as an infinite family of two-dimensional light distribution functions--one for every possible angle at which the incident bean can strike the surface element. This involves an overwhelming quantity of data, especially where high directional resolution is needed to describe glints and specularities. 


\section{APPENDIX C}

\section{COMPUTER PROGRAM FOR INVERSE SCATTERING PROBLEM}

The following is a computer program for calculating the effective transfer function, the rms surface roughness and the surface autocovariance function from scattered light data. Once these surface characteristics are known, the same program can be used to predict the scattering properties at a different wavelength. 


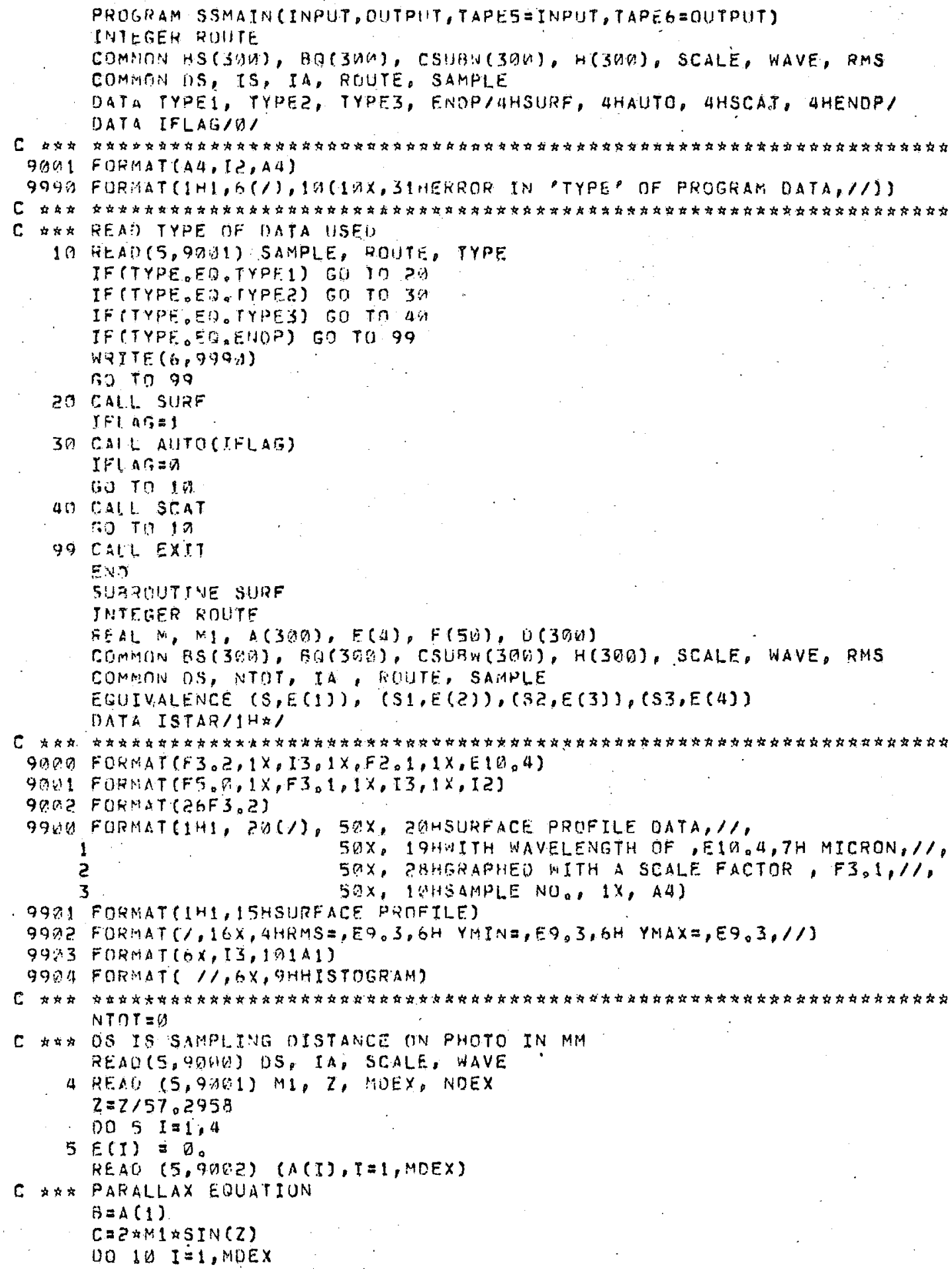




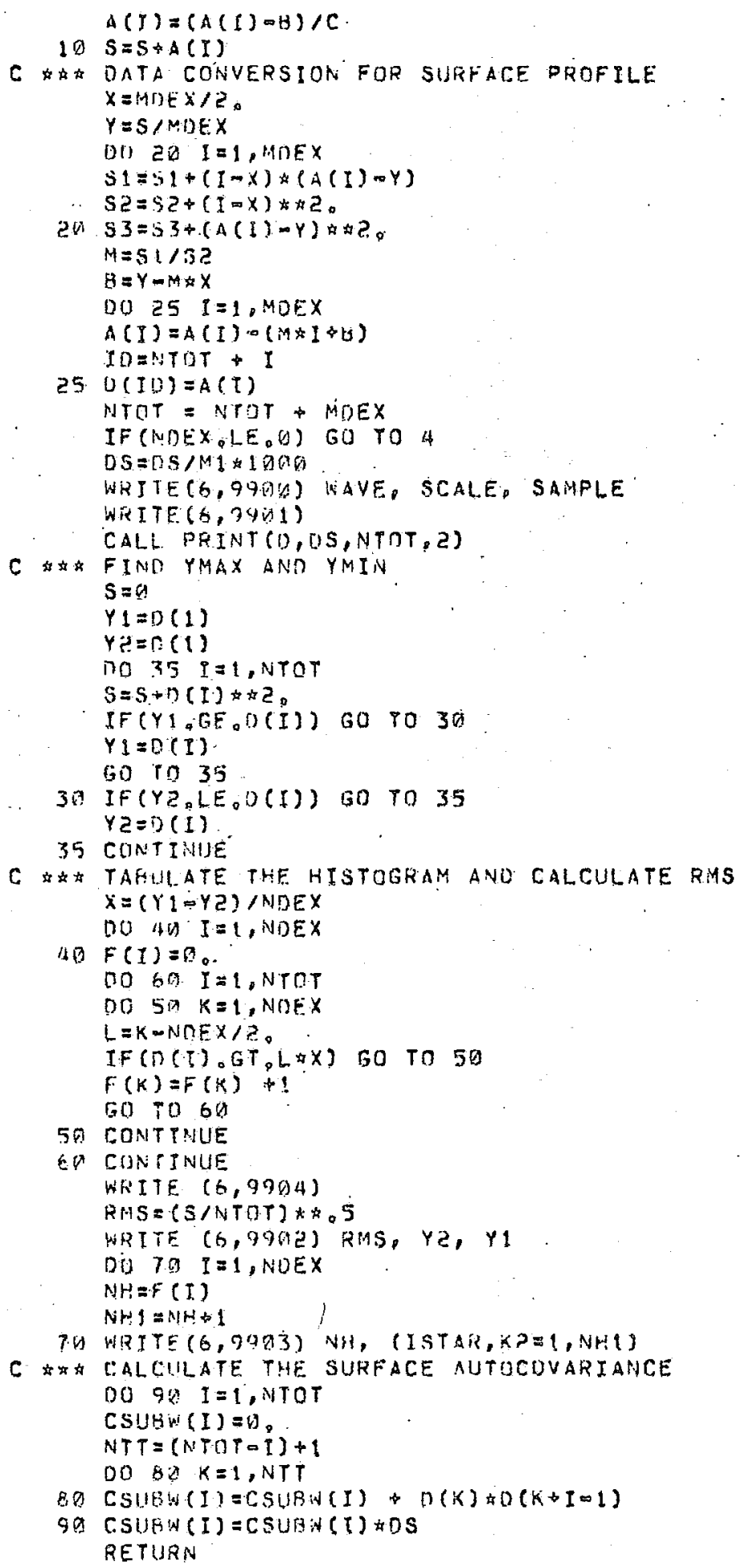




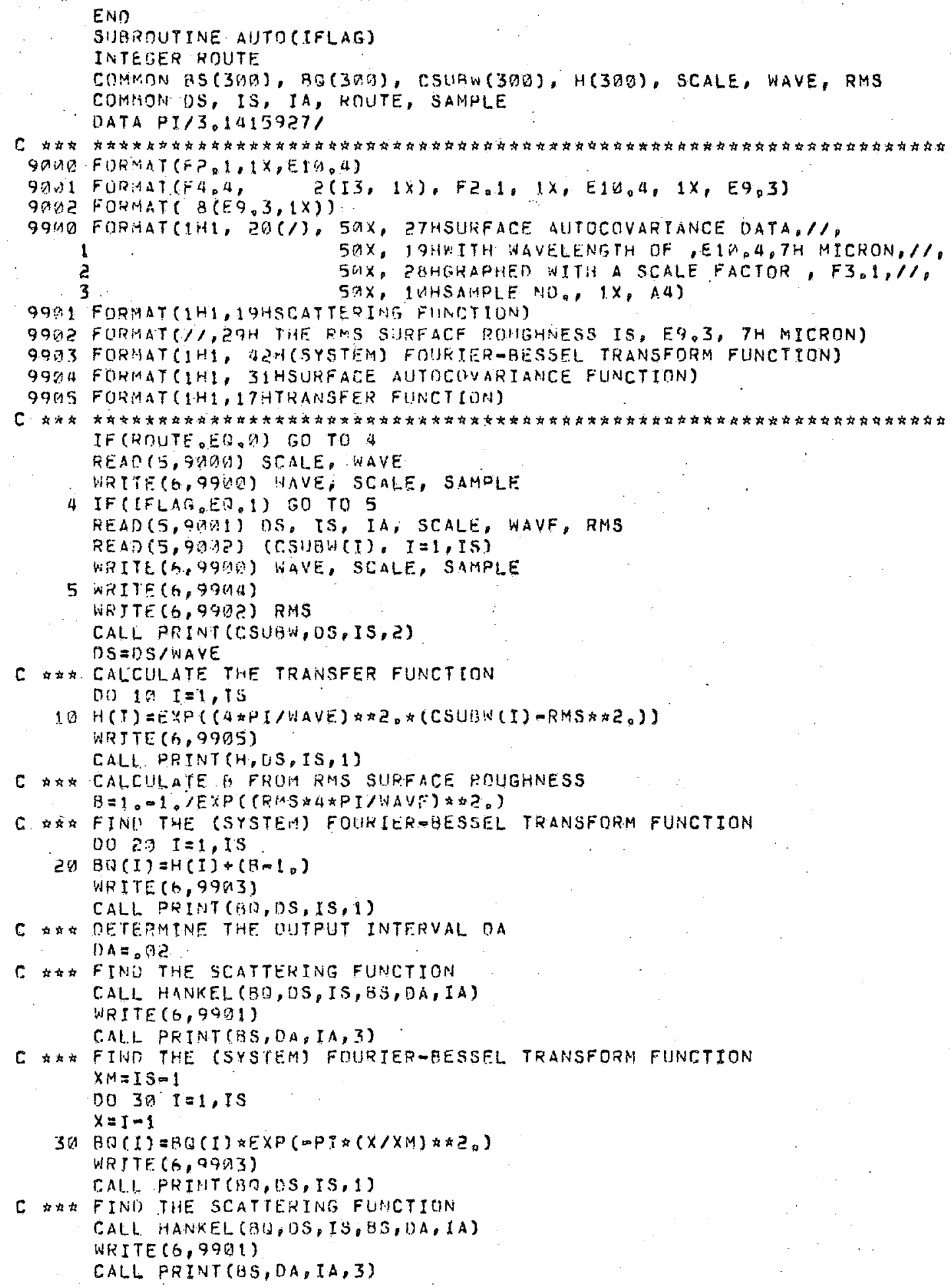




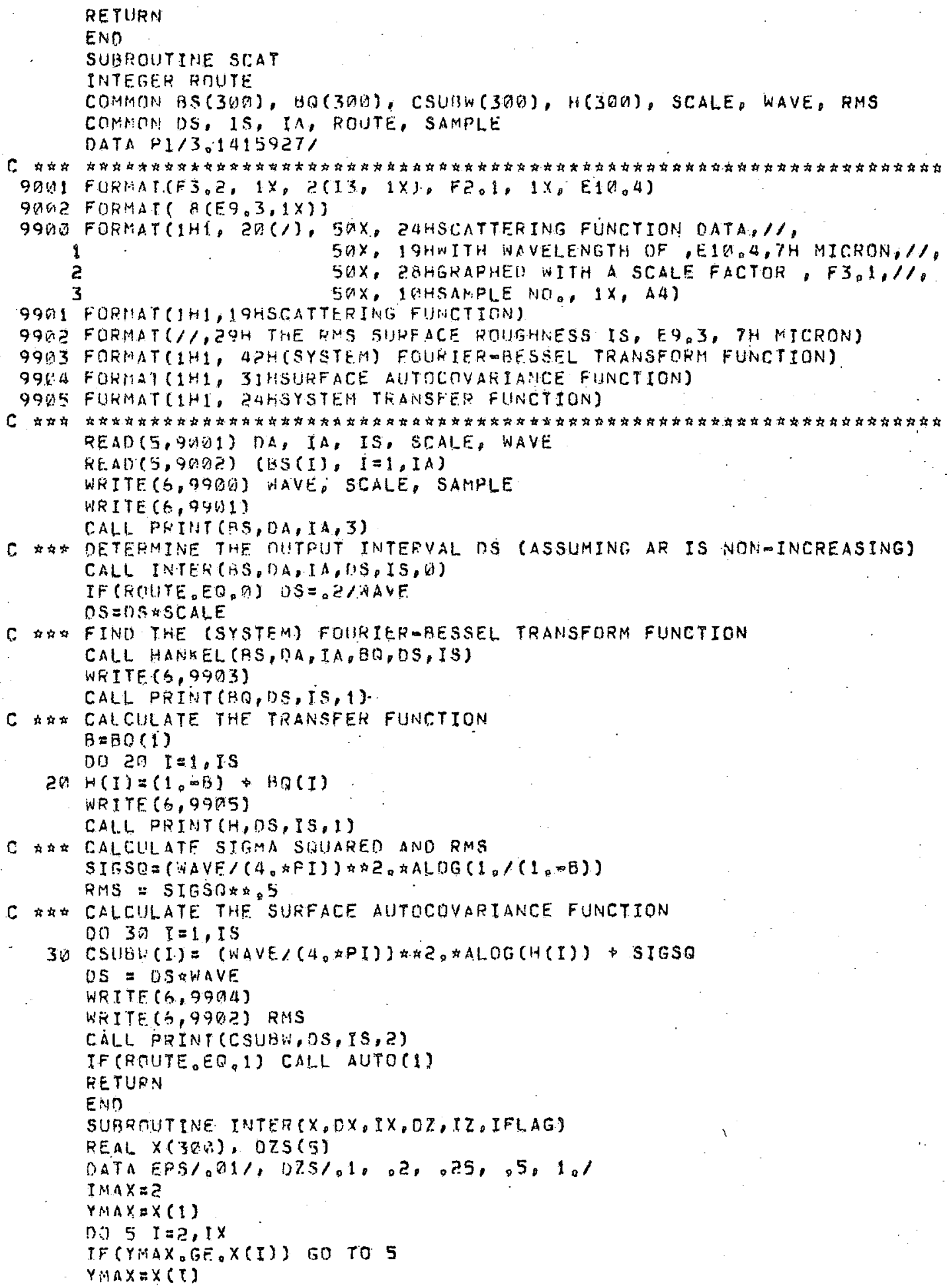




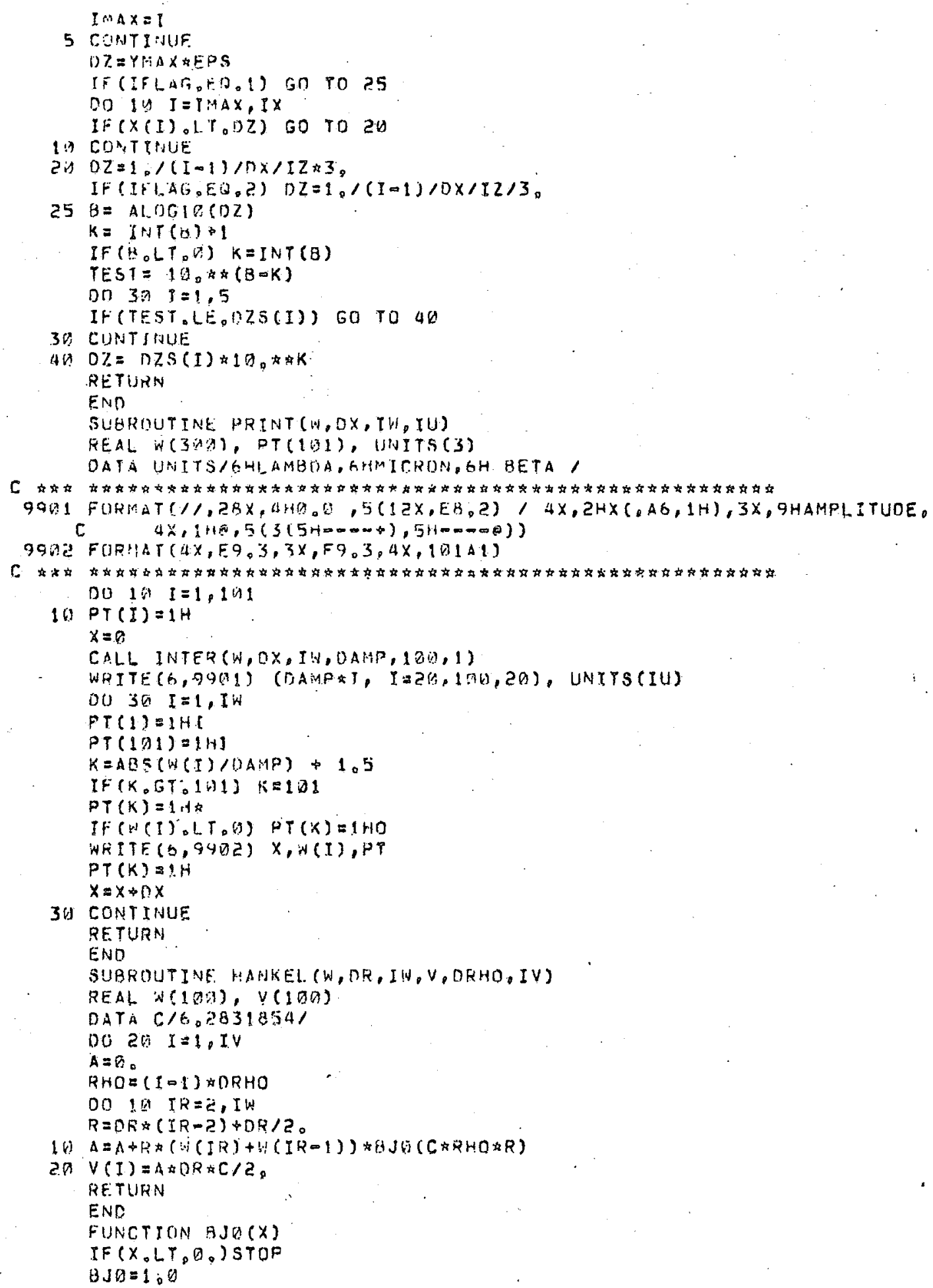


IF $\left(X_{0} E A_{0} Q_{0}\right)$ RETURN

CONST $=Z_{0} / X$

$M=18+1.3 * X$

$F M I=1.0 E=28$

$F M=0$ 。

$A L F=0$.

$M=(M / 2) \star 2 \& 1$

$M I=M-1$

$M 2=4$ 的 2

DO $2 \mathrm{i} K=1, M \mathrm{M}, 2$

$B M K=\{M-K j$ *F M $I$ CONST OFM

$F M=F M 1$

$F M I=6 \% M$

$B M K=(M 1-K) \& F M I+C O N S T-F M$

$F M=F M 1$

$F M I=\{3 M K$

$20 \quad A L F=A L F \nleftarrow B M K$

$A L F=? \cdot A * A L F=B M K$

$B J Q=R M K / A L F$

RETUKN

END 


\section{APPENDIX D}

COMPUTER PROGRAM FOR PROCESSING SURFACE

PROFILE DATA FROM ELECTRON-MICROGRAPH STEREOCOMPARATOR

The following is a computer program for processing surface profile data from the electron-micrograph stereocomparator. It provides the surface height distribution, the rms surface roughness, and the surface autocovariance function. 


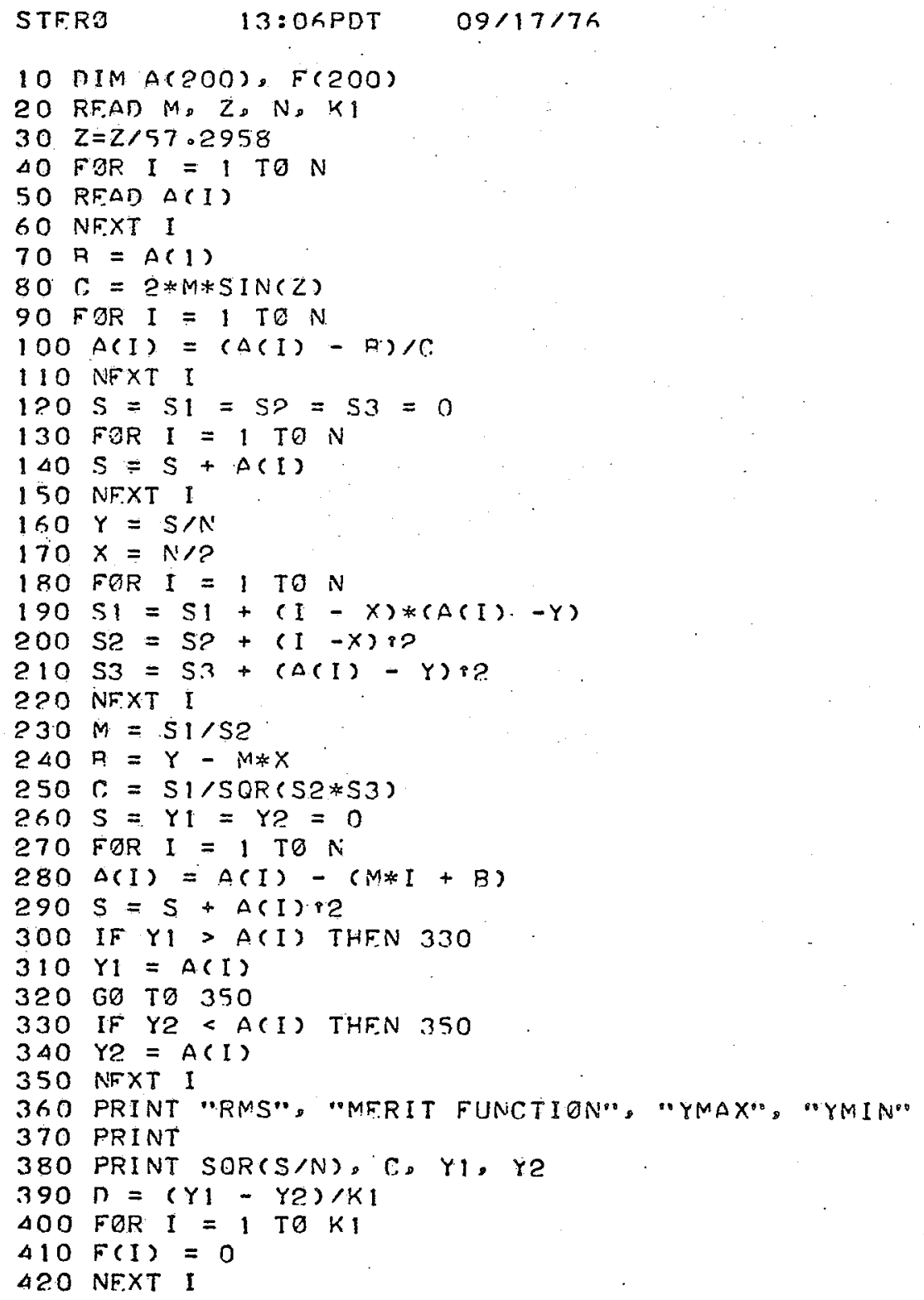




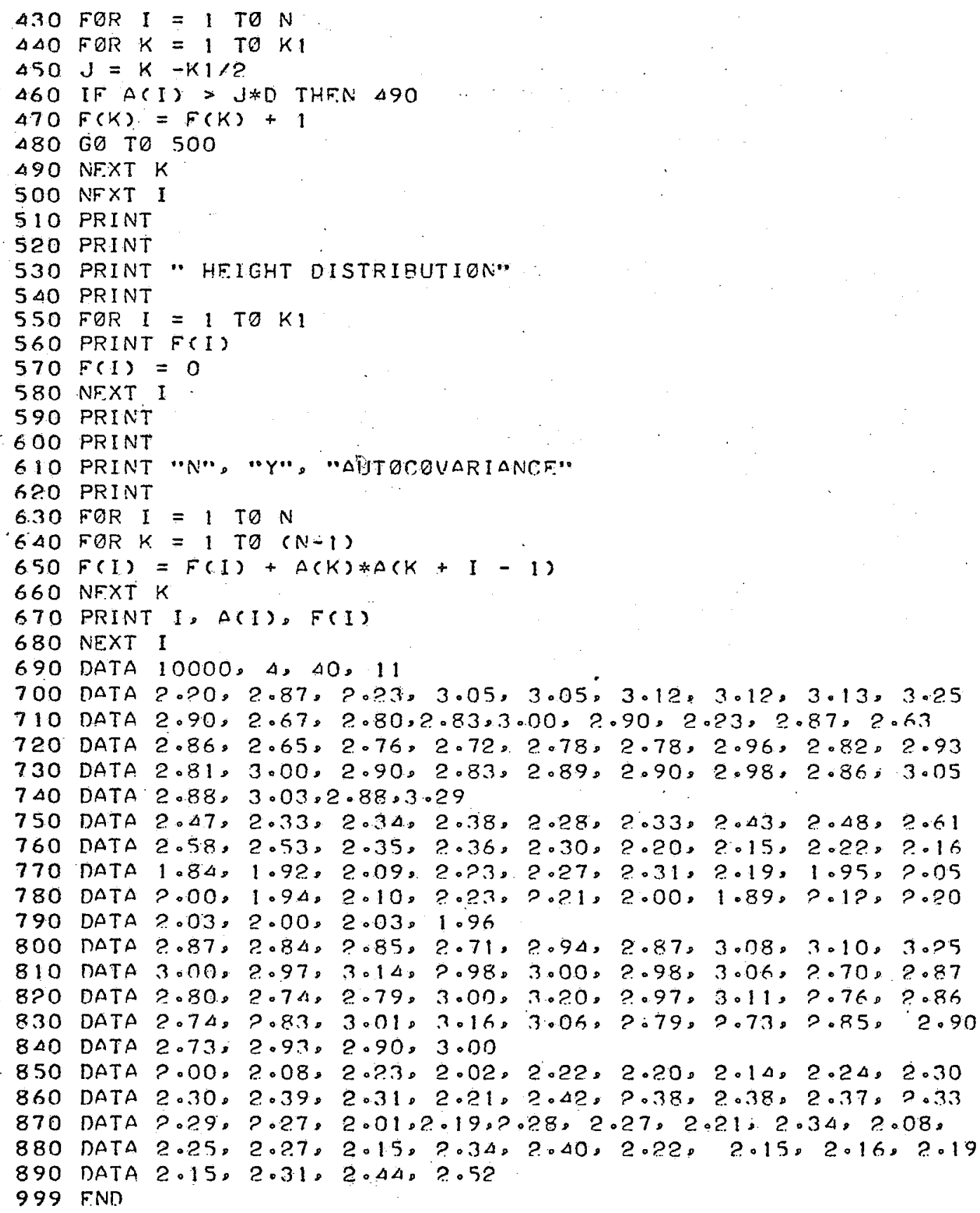




\section{APPENDIX E \\ DESCRIPTION OF SCANNING FECO INTERFEROMETER \\ USED FOR DETERMINING STATISTICAL \\ PROPERTIES OF OPTICAL, SURFACES}

The following is the manuscript of a paper presented at the 1974 Annual Meeting of the Optical Society of America in Houston, Texas (J. Bennett, 1974). 
Use of Interferometry for Determining the rms Roughness, Autocovariance Function, and Other Statistical Properties of Optical Surfaces*

Jean M. Bennett

Michelson Laboratory, Naval Weapons Center

China Lake, California 93555

\begin{abstract}
A FECO Scanning Interferometer will be described that can measure very small height differences with a lateral resolution of 2 microns to yield statistics for optical surfaces.
\end{abstract}

The other papers in this Symposium on Techniques in Surface Interferometry have mainly considered the use of interferometers to study the contours on optical surfaces, or more specifically the deviations from the desired surface contours. These deviations are sometimes called the figure of the surface, and opticians frequently talk of half-wave or quarter-wave optical surfaces when they mean that the deviations from a perfect plane or curved surface are one-half or one-quarter of the wavelength of the light used to test the surface (traditionally the mercury green line at $5461 \AA$ ). The figure of an optical surface is important in determining the resolving power, focusing properties, and aberrations in an optical system. For this reason much work has been devoted to interfacing the interferometer, which can sense figure errors, to the polishing machine which can eliminate them.

Optical technology has now progressed to the point where another parameter, the microroughness, also becomes important. Microroughness on the surface scatters some of the light into unwanted directions. Scattering cannot only reduce the contrast in optical images by removing light from the bright areas and filling in the dark ones, but it can also drastically reduce the optical throughput of a system long before the resolving power is affected. Scattering is also a serious problem when one is trying to observe a weak object that is very close to a bright object. This situation occurs frequently in astronomy when, for example, one is trying to observe details in the solar corona near an occulting disk, or when looking at a faint star located close to the moon or a bright star.

The problem of scattering from optical surfaces has been around for a long time. Even before 1900 Albert A. Michelson, the man best remembered for his measurements of the velocity of

*Reproduced here with permission of the author. 
light and the Michelson-Morley experiment, thought about light scattering. At the Michelson Museum. in China Lake we found an entry scribbled in one of Michelson's pocket notebooks along with shopping.lists, a prescription, notes for a coming lecture, possible causes of error in an experiment, and other diverse items. The entry is shown in Slide 1 and reads, "Find relation between roughness of surface and angle of scattering. Much has happened in the 80 or so intervening years, but we still have not satisfactorily solved the problem Michelson hastily noted down around 1890 .

What we have learned about the relation between surface roughness and scattered 1 ight is that the total hemispherical scatter from a surface (i.e., all the light scattered into a hemisphere) is related primarily to the heights of the surface irregularities when these heights are small compared to the wavelength of light. By measuring the total hemispherical scatter (frequently called TIS for short) and assuming a Gaussian distribution for the heights of the irregularities, we can obtain a value for the rms roughness of the surface. For many types of surfaces, particularly polished glass, fused quartz, calcium and magnesium fluorides, etc., this rms roughness value is in excellent agreement with the roughness value obtained from interferometric measurements, a technique $I$ wi 11 describe in detail in a few minutes. However, where the scattering theory falls short is in predicting the effects of scattered light from unusual optical surfaces such as polished alkali halides, polished metals, electropolished metals, and micromachined metals. In these cases TIS measurements can yield an effective rms roughness value that is either considerably smaller or considerably larger than the interferometrically measured value. More troubling even than this is the observation that we are not able to correctly predict the angular dependence of scattered light about the specular direction even for the smoothest polished glass surfaces... This situation arises because all the scattering theories assume a Gaussian autocovariance or autocorrelation function for the surface and none of the real surfaces we have studied have Gaussian autocovariance functions. For this reason, in order to have a theory which correctly predicts the effects of scattering from a surface, we need to know the autocovariance function and other statistics of the actual surface. How to measure these statistical properties is the subject I am going to discuss for the remainder of my talk.

Interferometry has been shown to be an excellent method for looking at very small height differences on surfaces, height differences of a few angstroms, i.e., a few thousandths of the wavelength of light. Tolansky pioneered this type of interferometric technique and gave the interference fringes the 
enigmatic name of Fringes of Equal Chromatic Order, or FECO for short. The two main things that are important about this type of intereference fringes are (1) that they contour height variations on surfaces, and (2) they occur as wiggly black.1ines in a continuous spectrum of light reflected from the interferometer. I am making the second point to distinguish FECO fringes from the more common Fizeau fringes which also contour irregularities on optical surfaces, but which are formed in monochromatic light. FECO fringes have advantages over Fizeau fringes in that the order of interference of the fringe is always known, and small areas of the surfaces can be studied at will without having to readjust the tilt of the interferometer plates.

I am now going to describe a FECO system we have built to measure the statistical properties of various types of optical surfaces and I will show you samples of the data we have obtained with this system. I will also mention some types of experiments we are planning for the future.

A photograph of the FECO Scanning Interferometer is shown in Slide 2. Most of the instrument consists of a signal averager, minicomputer and teletype, and the interferometer, the heart of the experiment, is the smallest part. The optical arrangement is shown in slide 3. The interferometer I consists of the sample to be studied, coated with an opaque layer of silver (upper plate) and a super smooth surface of polished fused quartz coated with a semi-transparent film of silver of approximately $95 \%$ reflectance. The two optical surfaces are very close together, being separated by only a few half wavelengths of light. The actual spacer consists of the dust particles on the two surfaces. The interferometer is illuminated in reflection by a collimated beam of white light from a xenon arc. The important feature of the FECO system is 1 ens $\mathrm{L}_{2}$ which focuses an image of the interferometer surfaces on the slit $S$ of a constant deviation spectrograph. Thus, the interference fringes which are viewed in the focal plane of the spectrograph contour the irregularities on the pair of optical surfaces. A picture of what might be observed is shown in the circular inset. Three mercury lines are included for wavelength calibration purposes, but the information about the surface topography is given in the wiggly interference fringe. There is a one-toone correspondence between the wiggles on the interference fringe and height variations on the pair of optical surfaces. To get an idea of the magnitudes of the quantities we are dealing with, the wavelength variations can yield information about variations of the heights of irregularities of the order of a few angstroms. The lateral resolution is much smaller, so that the length of the interference fringe corresponds to a distance of one $\mathrm{mm}$ on the interferometer surface. The width of the 
spectrograph slit determines the other dimension, so we are actually looking at an area one mm long by about 2 microns wide.

To obtain the statistics for the section of the interferometer surface we are observing, we scan the spectrum line by line using a special slow scan TV camera. After suitable ageraging, the information on one scan line appears as shown in the upper right hand part of the slide. The desired information is the wavelength of the portion of the interference fringe included in the scan line and this is obtained from the computer analysis. The information from the entire frame consists of wavelengths of 512 equally spaced points on the interference fringe, so we have data for 512 different areas on the surface, each one of which is a little square 2 microns on a side. In the statistical analysis a least squares quadratic curve is calculated from al1 512 wavelengths and defines the mean surface level. Then wavelength differences from this curve are converted into height differences above and below the mean surface leve1. Using the height differences we can determine the rms roughness, height distribution function, slope distribution function, rms slope, autocovariance function, and other statistical parameters for the surface.

I am now going to show some data that are typical of what we have obtained for various types of surfaces. We have studied very smooth glass-type surfaces such as fused quartz, Cervit, calcium fluoride and magnesium fluoride, polished alkali halides (potassium chloride and sodium chloride), polished metals (copper, beryllium copper, titanium and titanium alloys, molybdenum, and stainless stee1), electropolished nickel, machined copper, and holographic gratings. A1I of the smoothest polished glass-type materials have similar statistics, and Slide 4 shows results for one of these. This is an extremely. smooth calcium fluoride surface polished by Abe Klugman of the Northrop Corporation and had a visually measured roughness of $9.4 \AA$ rms: At the top of the slide is a Polaroid photograph of the interference fringe and directly below it is a TV scan of the center line of the fringe. Note that the wiggles on the fringe represent height differences of considerably less than $10 \AA$. The autocovariance function shown below can be roughly considered as the correlation between points on the surface separated by the amount shown on the $x$ axis. For the very smooth surfaces there is positive correlation between closely spaced points but those farther away are random.

The height and slope distribution functions for the same surface are shown on Slide 5. Note that both measured distribution functions (the histograms) are very close to Gaussian, 
and actually the smooth curves are Gaussians having the same areas under the curves as do the measured ones. The slope distribution function is only half a Gaussian curve because we do not distinguish between positive and negative slopes.

Polished alkali halide surfaces and some polished metal surfaces are similar in that both are composed of macro and microscratches with no smooth areas in between. In S1ide 6 we see the scanning camera trace for a polished $\mathrm{KCl}$ surface and the autocovariance function. Note the oscillations in the autocovariance function which indicate longer range correlations than those observed for the smoothest surfaces. Note also that neither this autocovariance function nor the preceding one were Gaussian in shape. In fact, we have never observed an autocovariance function that did have a Gaussian shape. In S1ide 7 we see that there is slight asymmetry in the height distribution function although the slope distribution function seems to be a very good Gaussian. Incidentally, I should point out that the rms roughness values shown on the height distribution function. histograms are about a factor of two smaller than the visually measured values. This is because the scanning camera does not take into account the width of the fringe, only its center line. Visually we measure the extreme width of the fringe and convert the peak-to-valley roughness to an rms value by dividing by $2 \sqrt{2}$. There is a very good 1inear relationship between visually measured roughnesses and the values obtained from the scanning camera.

In Siide 8 we see data for molybdenum, a typical polished metal surface. The autocovariance function with the oscillations is similar to that for KCl. The height and slope distribution functions shown in Slide 9 are reasonably good Gaussians although there is some raggedness on the height distribution function.

Recently very low scatter electroless nickel mirrors have become available. In slide 10 is shown the autocovariance function for one of these, which had a visually measured roughness of $23.6 \AA$ rms. This surface is a gradually undulating one with almost no obvious scratches. The autocovariance function also has a lower frequency oscillation than was observed for the KCl and molybdenum surfaces. In Slide 11 we see that there is a definite asymmetry in the height distribution function. There are proportionately more small bumps on the surface than there are small holes. However the slope distribution function is an extremely good Gaussian.

One of the most interesting surfaces we have encountered are those made on a special type of lathe by a single point 
diamond tool. Recently we determined the statistics on one which had a visual roughness value of only $22.5 \AA$ rms. S1ide 12 shows that the autocovariance function for this surface has only a long period oscillation. We probably are not resolving the individual grooves made by the diamond, but only multiples thereof. The height and slope distribution functions on. Slide 13 are reasonably good Gaussians even though there must be obvious periodicity in the surface.

As a final set of statistics, we see in Slide 14 a tracing of a holographic grating with a nominally sinusoidal groove shape formed in a photographic emulsion. This sample was kindly furnished to us by John Stover of Dow Chemical Company, Rocky Flats, Colorado. The autocovariance function is for only one scan and clearly shows the periodicity of the surface. The height distribution function on Slide 15 is clearly not Gaussian, and I did not even attempt to put a Gaussian curve through the data.

Our plans for the future include increasing the sensitivity of the scanning camera so we can obtain scans from many more portions of the surface. Most of the data shown here are averages of 8-10 separate scans. We also hope to automate the scanning interferometer so that we can make equally spaced scans adjacent to each other to obtain statistical data on a square one $\mathrm{mm}$ on a side.

In conclusion; I have described a type of interferometer which can be used to obtain statistical information about the topography of optical surfaces. This instrument can distinguish height differences of only a few angstroms and has a lateral resolution of about 2 microns. Using the statistics of the surface, we hope to be able to predict the scattering properties of the surface. 


Reproduction of an orlgial 1 tem
1n the collection of
DOROTHY MICHEISON LIVINGSTON

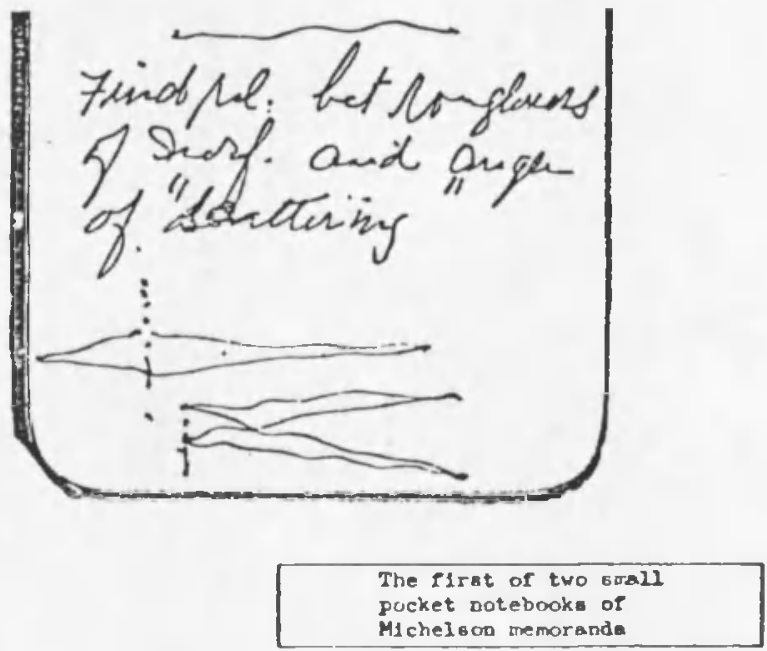

SLIDE 1

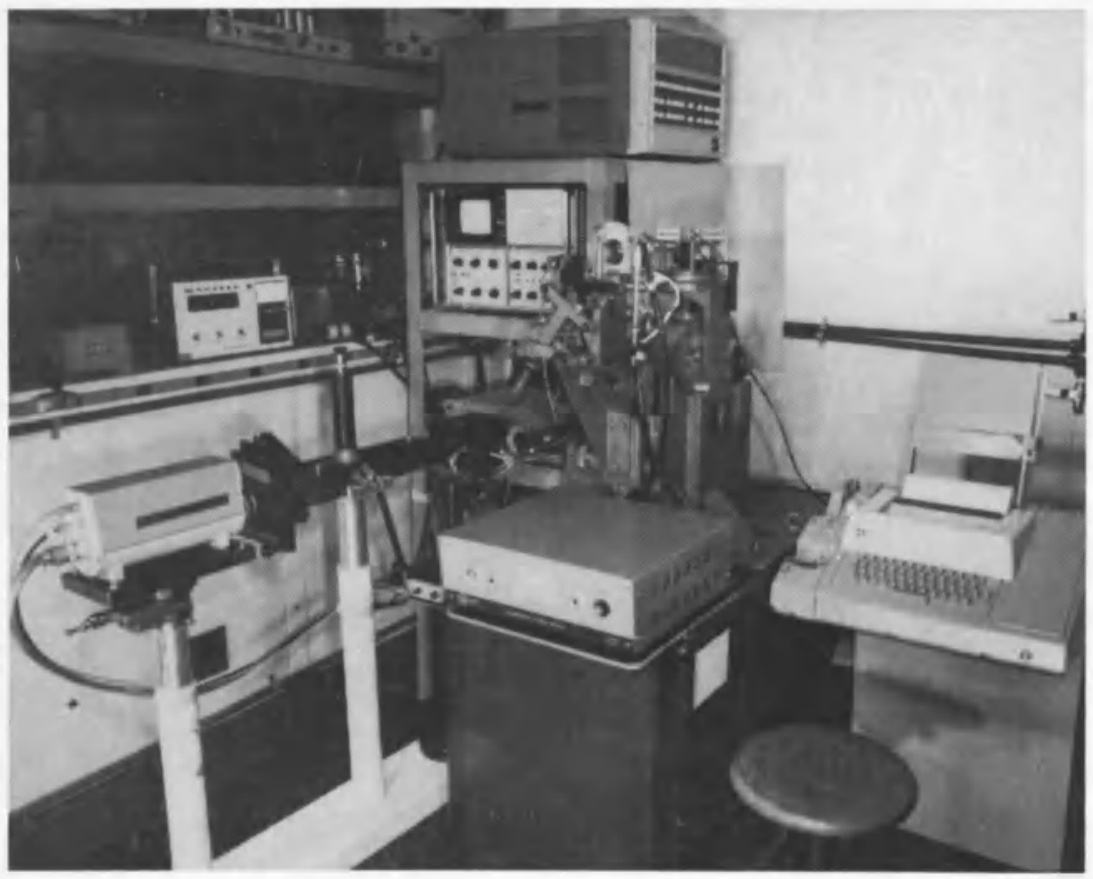


FECO INTERFEROMETRIC SURFACE SCANNER

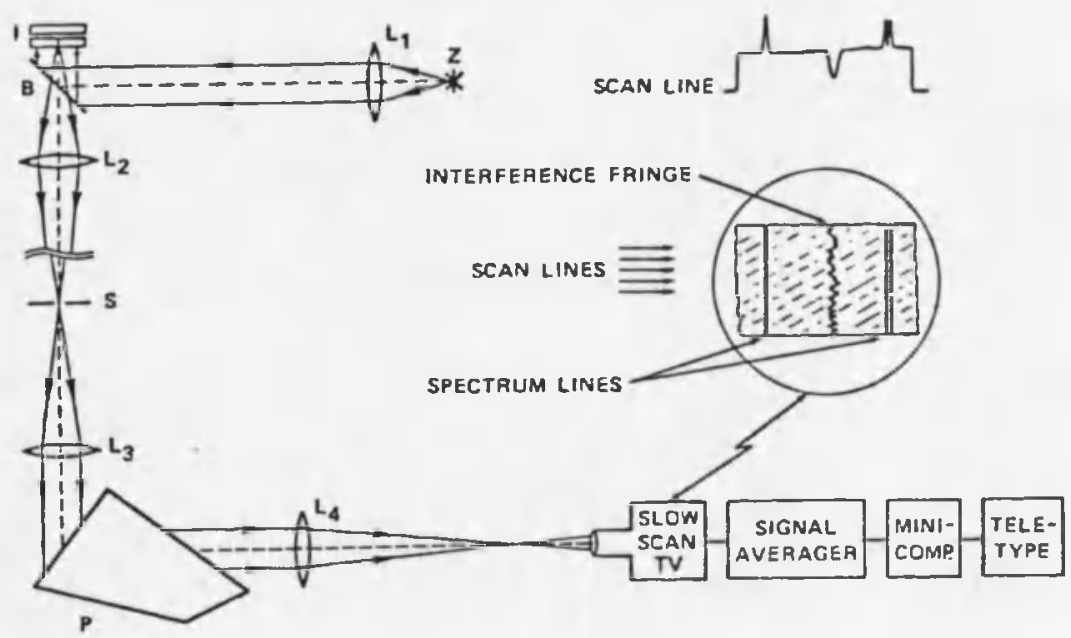

SLIDE 3

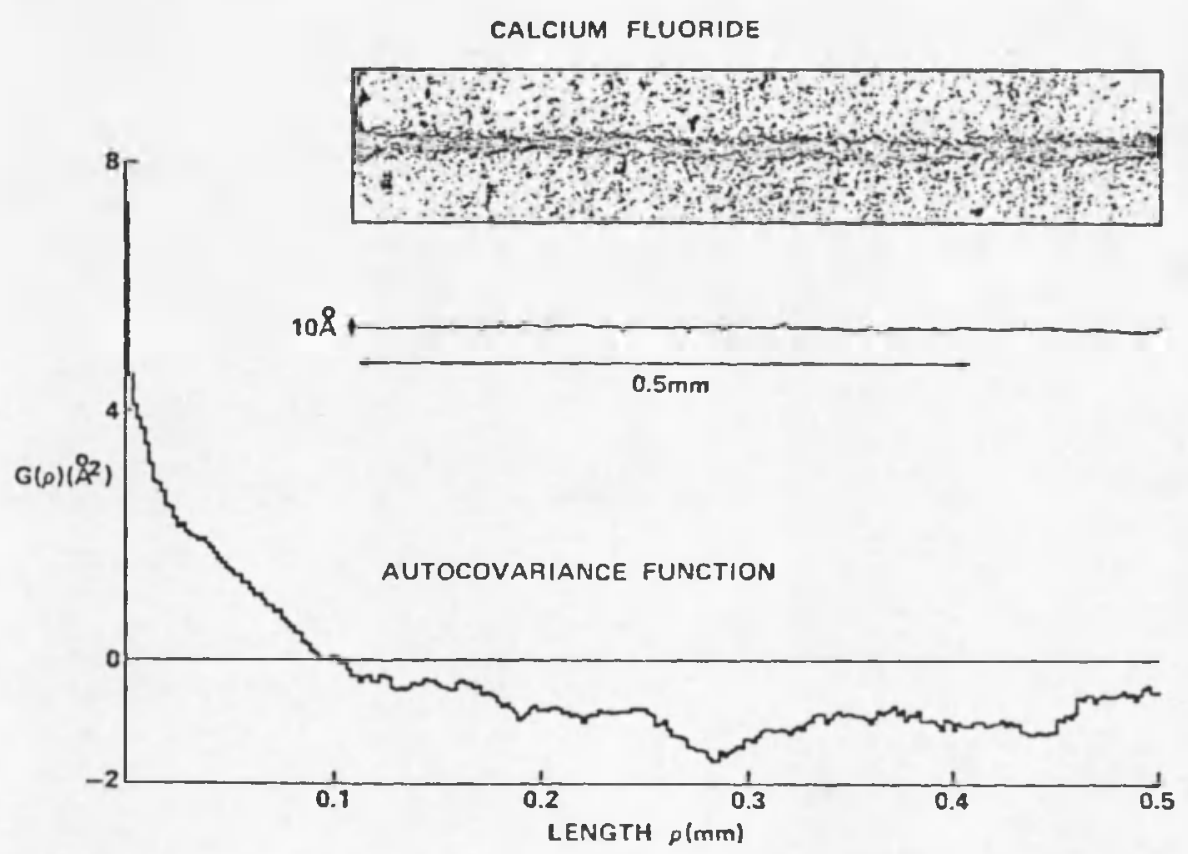



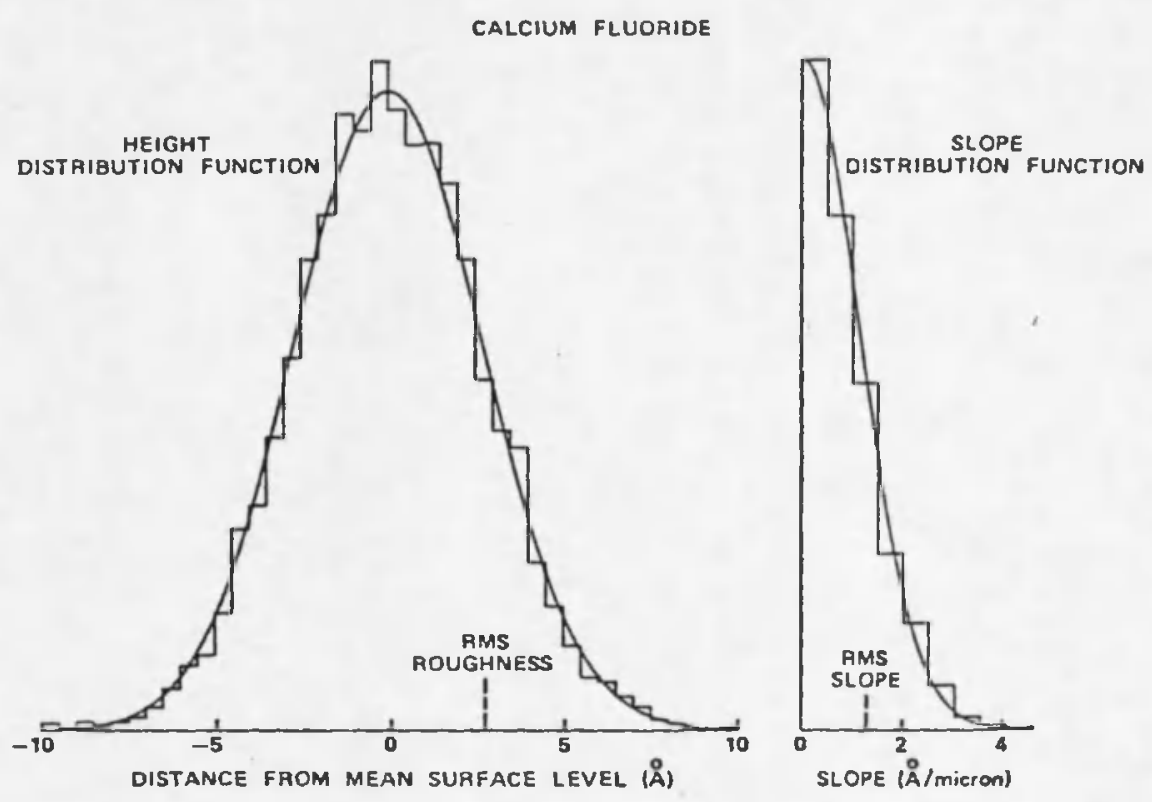

\section{SLIDE 5}

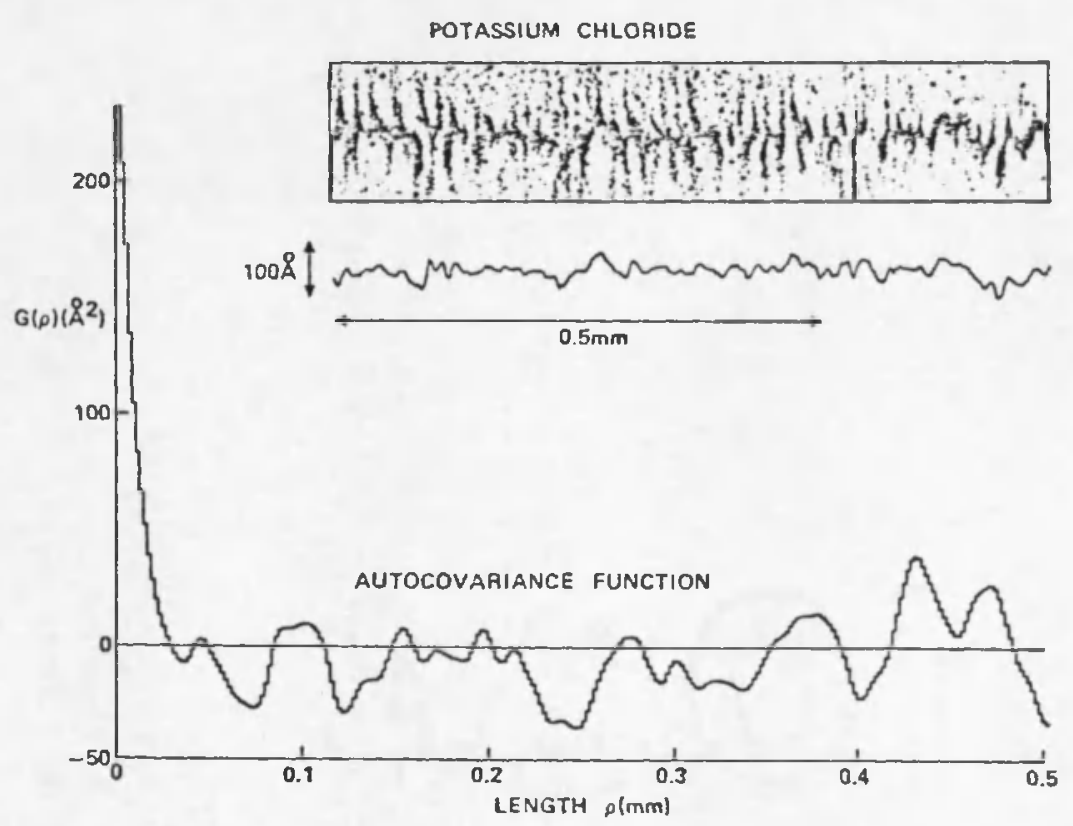



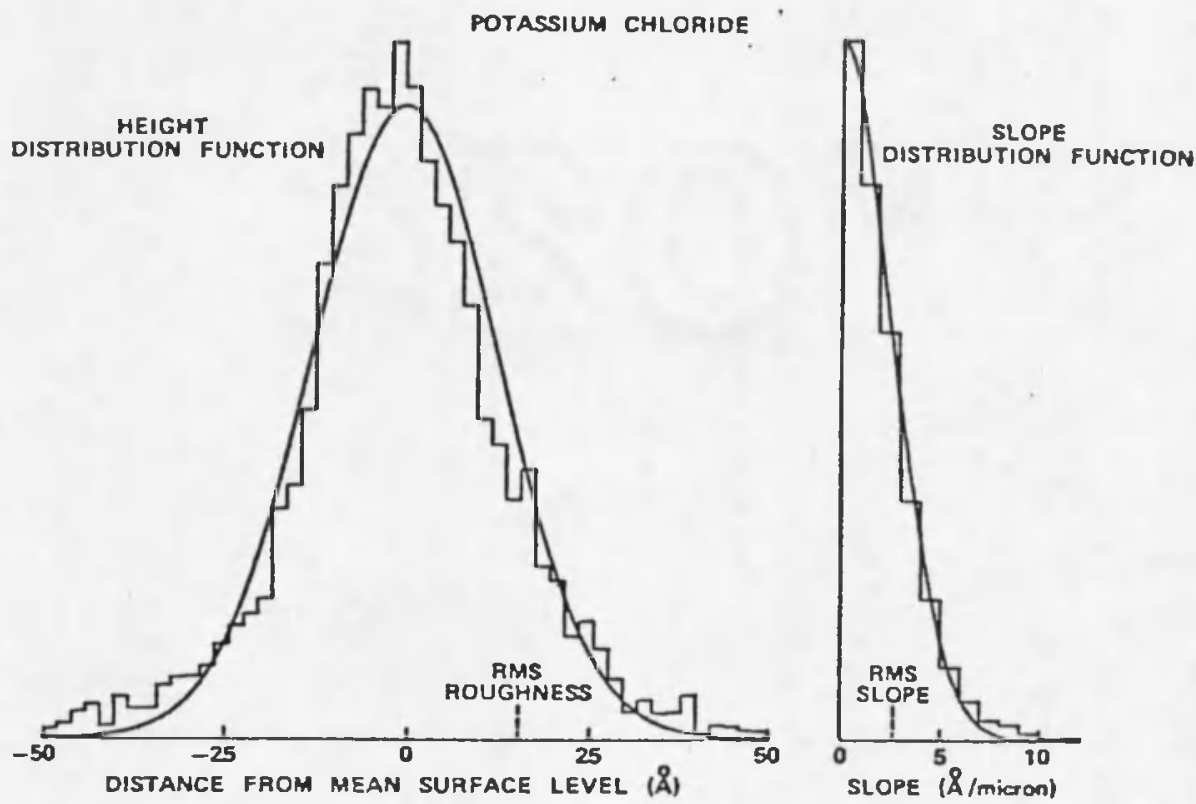

SLIDE 7

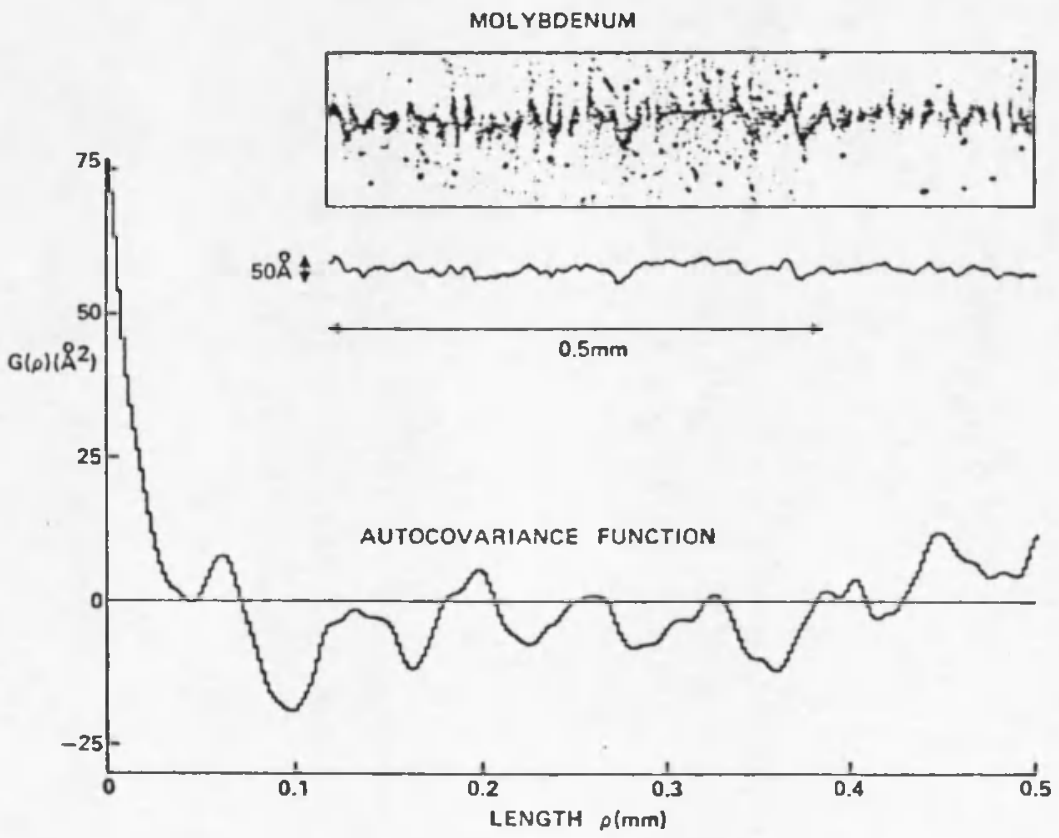



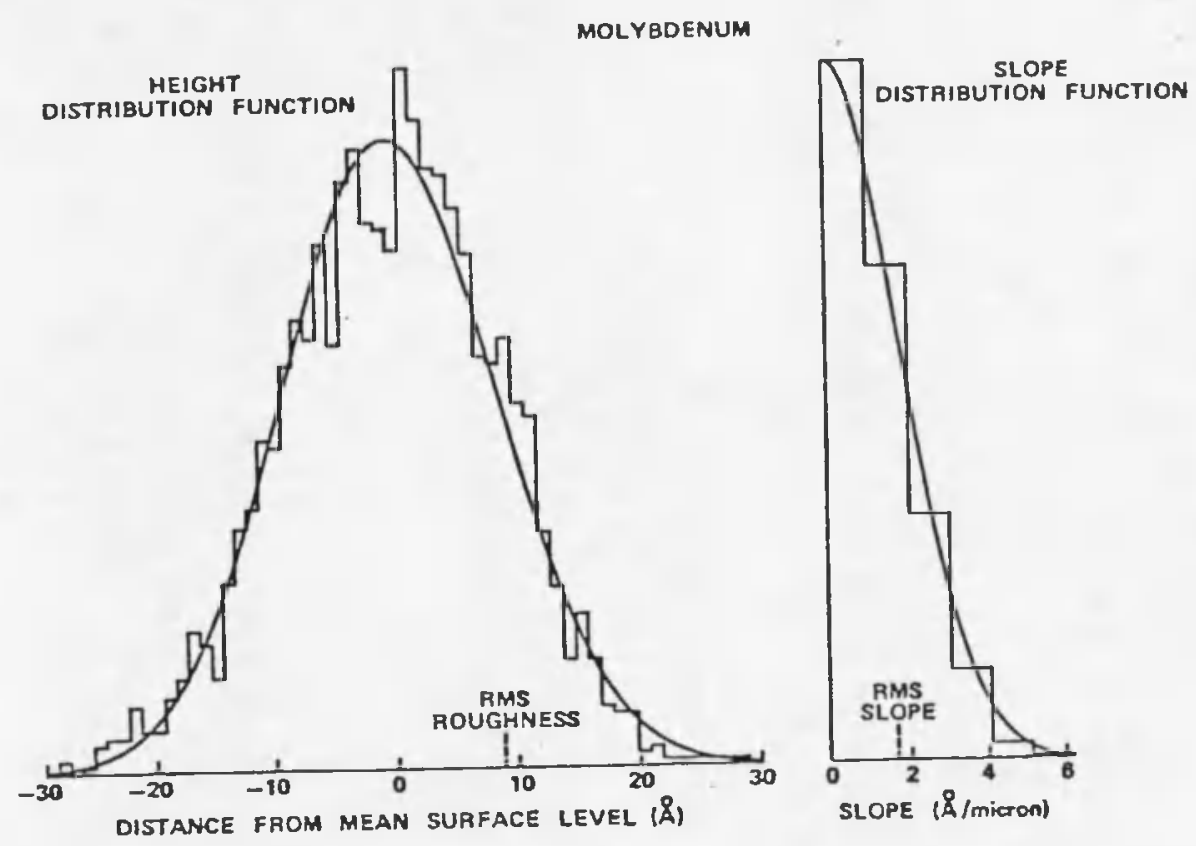

SLIDE 9

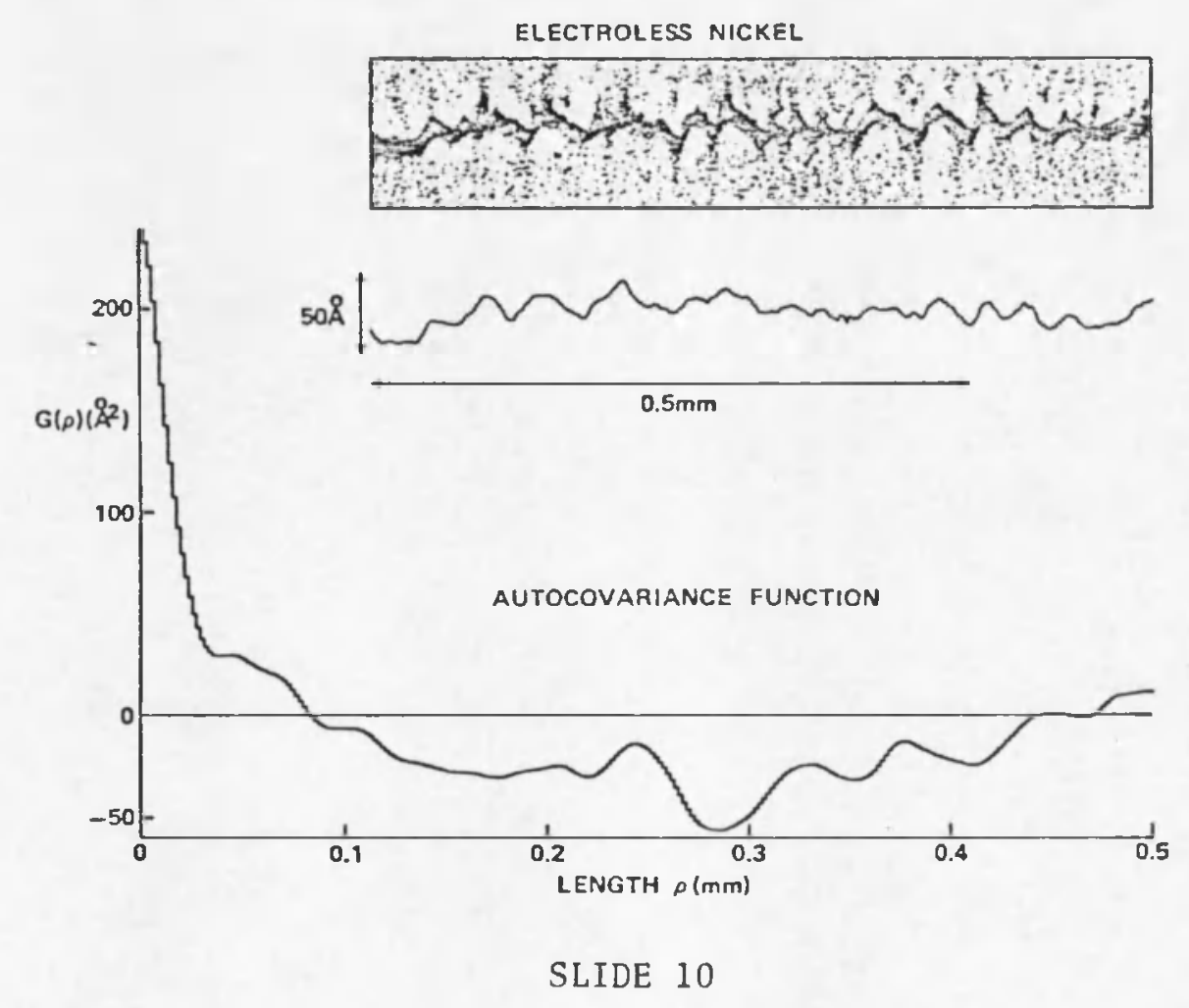



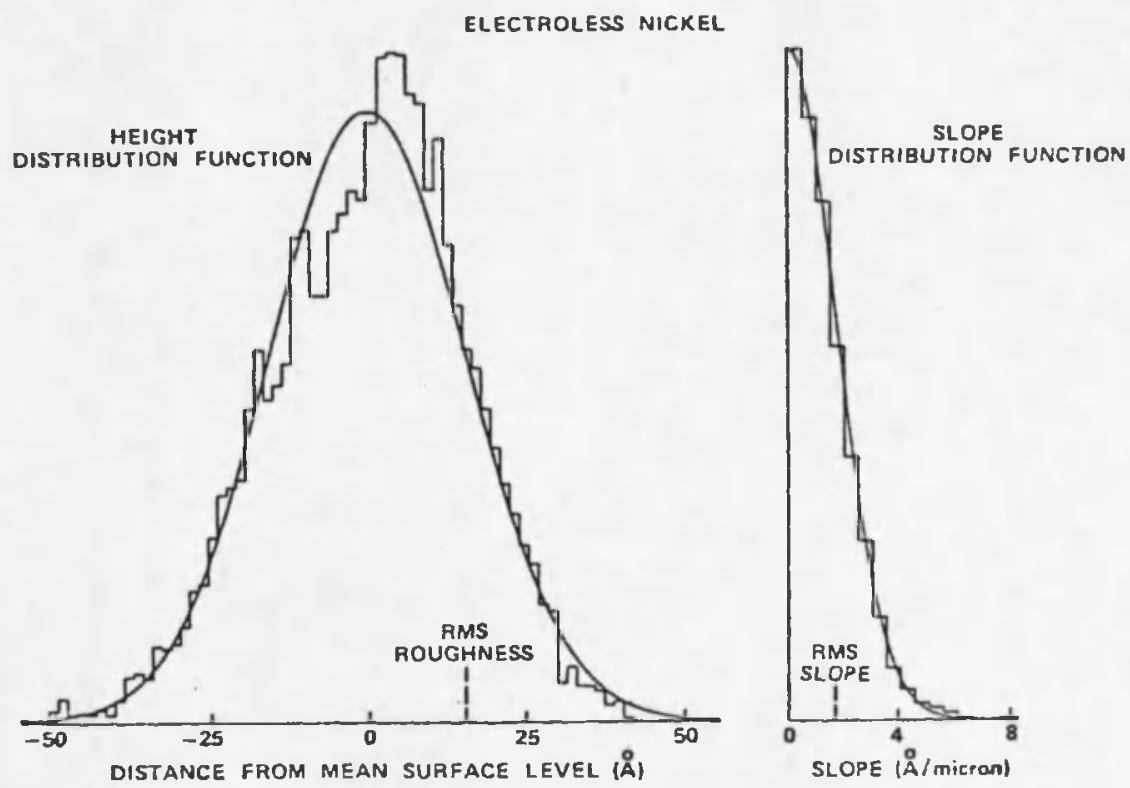

SLIDE 11

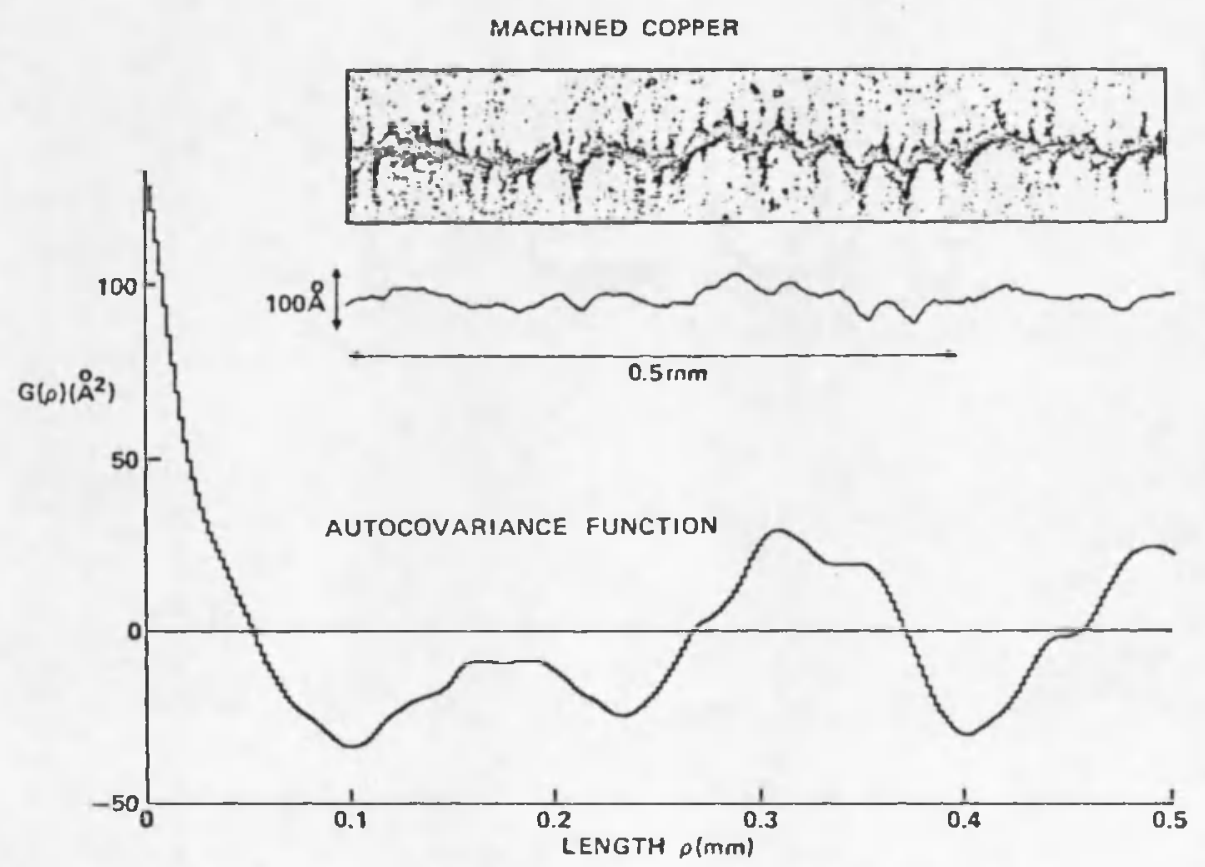



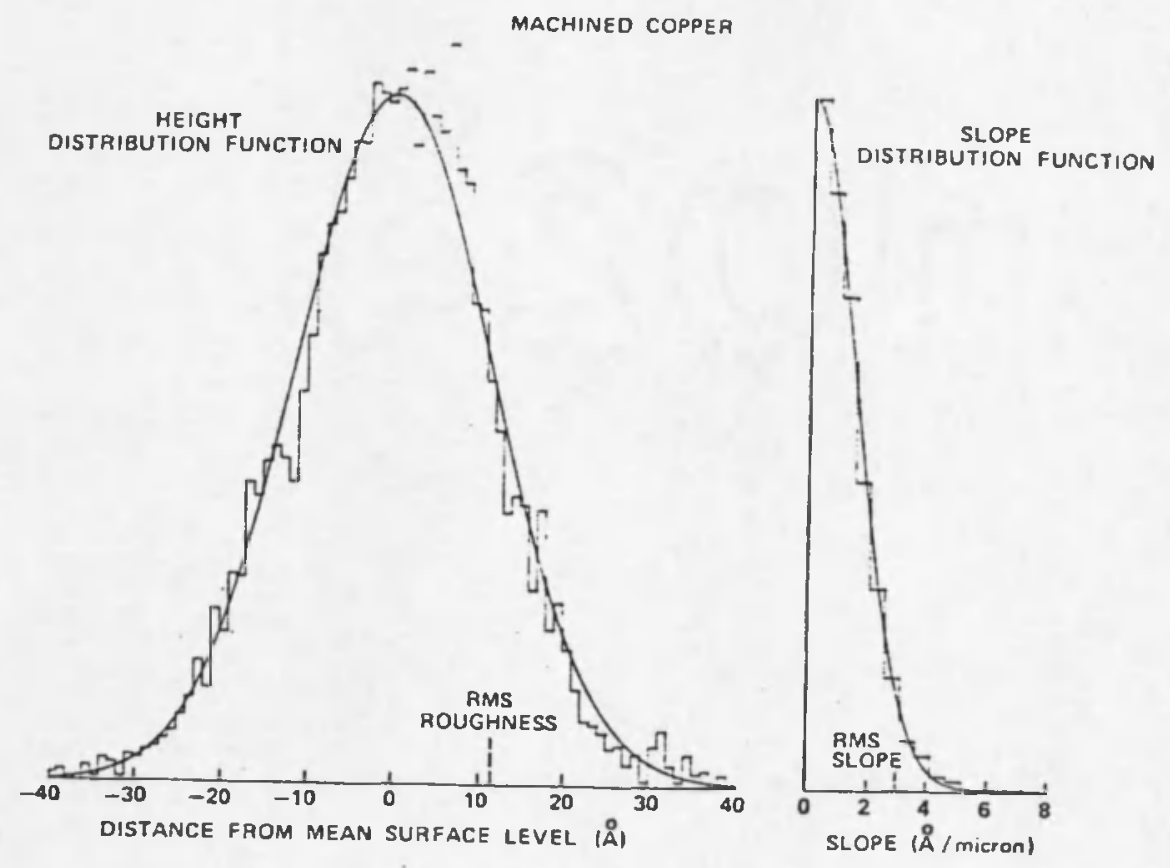

SLIDE 13

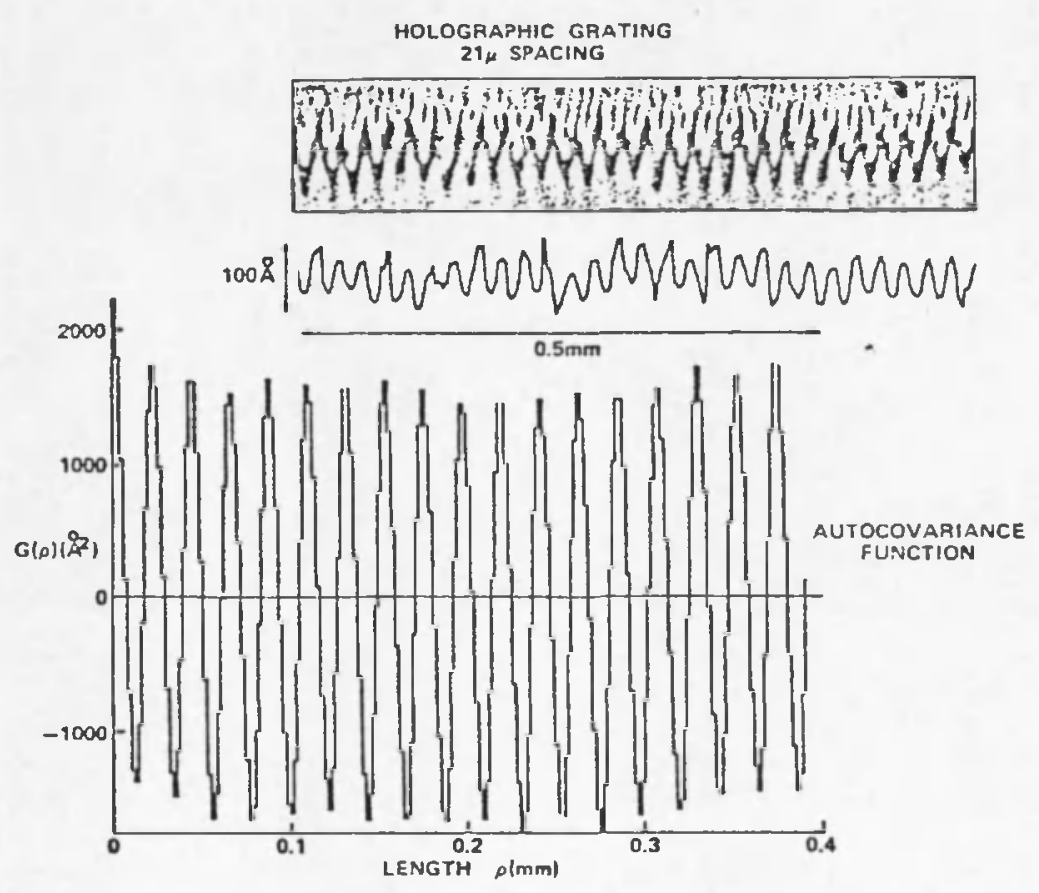




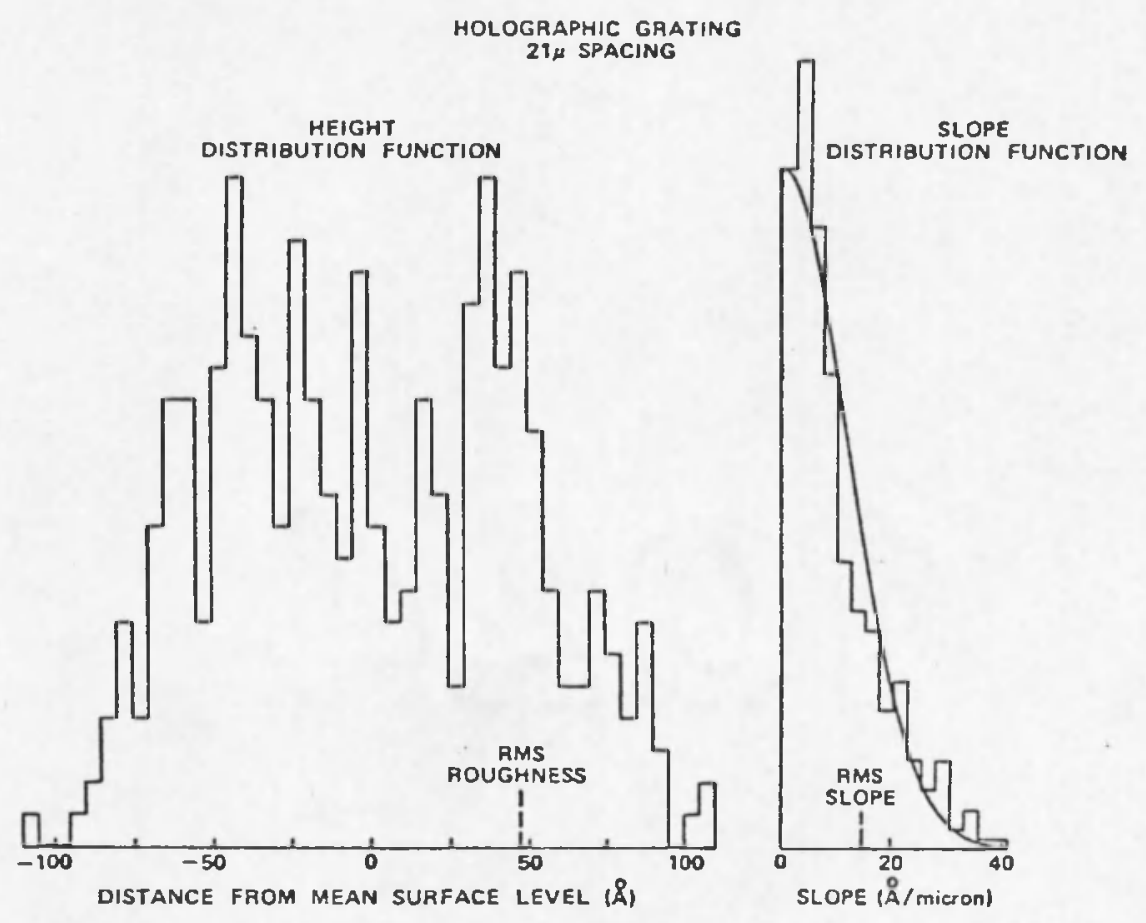

SLIDE 15 
LIST OF REFERENCES

Ament, W. S., "Toward a Theory of Reflection by a Rough Surface," Proc. IRE 41:142 (1953).

Ament, W. S., "Reciprocity and Scattering by Certain Rough Surfaces," IRE Trans. AP-ㅇ: $167-174$ (1960).

Barrick, D. E., "Two Experiments Yielding Lunar Surface Information Employing Polarized Radar Waves," Ohio State University Research Foundation, Columbus, Ohio, Available from the National Technical Information Service, No. N66-14962 (1965).

Barrick, D. E., "Rough Surfaces," Radar Cross Section Handbook,

Vo1. 2, G. T. Ruck, et al., eds., Plenum Press; N. Y., pp. 671-772 (1970) .

Bass, F. G., "Very High Frequency Radiowave Scattering by a Disturbed Sea Surface," IEEE Trans. AP-16:554-568 (1968).

Beaglehole, D., "Optical Excitation of Surface Plasmons," IEEE Trans. ED-17:240-244 (1970).

Beaglehole, D., and O. Hunderi, "Study of the Interaction of Light with Rough Metal Surfaces," Phys. Rev. B 2:309-329 (1970).

Beard, C. I., "Coherent and Incoherent Scattering of Microwaves from the Ocean," IRE Trans. AP9: 470-483 (1961).

Beckmann, P., "A New Approach to the Problem of Reflection from a Rough Surface," Acta. Tech. CSAV. 2:311-355 (1957).

Bennett, H. E., "Specular Reflectance of Aluminized Ground Glass and the Height Distribution of Surface Irregularities," J. Opt. Soc. Am. 53:1389-94 (1963).

Bennett, H. E., and J. M. Bennett, "Precision Measurements in Thin Film Optics," in Physics of Thin Films, G. Hass and R. E. Thun, eds., Academic Press, N.Y., Vol. 4, pp. 1-96 (1967).

Bennett, H. E., and J. O. Porteus, "Relation between Surface Roughness and Specular Reflectance at Normal Incidence," J. Opt. Soc. Am. 51: $123-9$ (1961).

Bennett, J., "Use of Interferometry for Determining the rms Roughness, Autocovariance Function, and other Statistical Properties of Optical Surfaces," (Abstract) J. Opt. Soc. Am. 64:1362 (1974). 
Blake, L. V., "Reflection of Radio Waves from a Rough Sea," Proc. IRE 38:301-304 (1950).

Bloembergen, N., and Y. R. Shen, "Coupling of Light with Phonons, Magnons, and Plasmons," Phys. of Quantum Elect. Conference Proc., P. L. Kelley, et al., eds., San Juan, Puerto Rico, McGraw-Hill, N. Y., pp. 119-128 (1965).

Bojarski, N. N., "Electromagnetic Inverse Scattering," Mathematics of Profile Inversion Workshop, Ames Research Center, Moffett Field, California, L. Colin, ed., pp. 6-34 to 6-37, NASA TM X-62, 150, Available from the National Technical Information Service, No. N73-11615, (1971).

Bracewel1, R. N., The Fourier Transform and its Applications, McGraw-Hi11, N. Y. (1965).

Breault, R. P., and B. B. Fannin, Optical Sciences Center, University of Arizona, Tucson, Private Communication (1973).

Chenmoganadam,.T. K., "On the Specular Reflection from Rough Surfaces," Phys. Rev, 13:96-101 (1919).

Church, E. L., and J. M. Zavada, "Residual Surface Roughness of Diamond-Turned Optics," App1. Opt. 14:1788 (1975).

Crowe11, J., and R. H. Ritchie, "Surface Plasmon Effect in the Reflectance of a Metal," J. Opt. Soc. Am. 60:974-799 (1970).

Curcio, M. E., "Evaluation of Low Scatter Technology for Aspheric Metal Mirrors," Oral Presentation at SPIE's 19th Annual International Technical Symposium, August 1975, San Diego, CA (1975).

Daniels, F. B., "A Theory of Radar Reflection from the Moon and Planets," J. Geophys. Res. 66:1781-1788 (1961).

Daudé,.A., A. Savary, and S. Robin, "Effects of Different Roughnesses on the Excitation by Photons of the Surface Plasmon of Aluminum," J. Opt. Soc. Am. 62:1-5 (1972).

Davies, H., "The Reflection of Electromagnetic Waves from a Rough Surface," Proc. IEEE 101:209-14 (1954).

DeBe11, M. A., and J. E. Harvey, "Recent Developments in Surface Scatter Studies," Abstract, J. Opt. Soc. Am 64:1404 (1974).

Decker, D. L., H. E. Bennett, and J. M. Bennett, "Light Scattering and Absorption in Infrared Laser Mirrors," Oral Presentation, SPIE's 19th Annual International Technical Symposium, August, 1975, San Diego, California (1975). 
Eckart, C., ".The Scattering of Sound from the Sea Surface," J. Acoust. Soc. Am. 25:566-570 (1953) .

Elson, J. M., and R. H. Ritchie, "Photon Interactions at a Rough Metal Surface," Phys. Rev. B 4:4129-4138 (1971).

Evans, J. V., and G. H. Pettengi11, "The Scattering Behavior of the Moon at Wavelengths of 3.6, 68, and 784 Centimeters," J. Geophys. Res. 68:423-447 (1963).

Feinstein, J., "Some Stochastic Problems in Wave Propagation," Part I. Trans. IRE AP 2:23-30 (1954).

Fuks, I. M., "Theory of Radio. Wave Scattering at a Rough Sea Surface," Soviet Radiophysics 9:513-519 (1966).

Fung, A. K., "Character of Wave Depolarization by a Perfect1y Conducting Rough Surface and Its Application to Earth and Moon Experiments," Planetary and Space Science 15:1337-3147 (1967).

Fung, A. K., and R. K. Moore, "Effects of Structure Size on Moon and Earth Radar Returns at Various Angles," J. Geophys. Res. 69:1075-1081 (1964).

Goodman, J.W., Introduction to Fourier Optics, McGraw-Hill, San Francisco, CA (1968).

Hagfors, T., "Backscattering from an Undulating Surface with Applications to Radar Returns from the Moon," J. Geophys. Res. 69:3779-3784 (1964).

Hopkins, H. H., Wave Theory of Aberrations, Oxford Clarendon Press, London $(1950)$.

Isakovich, M. A., "The Scattering of Waves from a Statistically Rough Surface," Zhurn. Eksp. Theor. Fiz. 23:305-314, Translation available from Defense Scientific Information Service, DRB Canada, T 112 R (1952).

Judd, D: B., "Terms, Definitions, and Symbols in Reflectometry," J. Opt. Soc. Am. 57:445-452 (1967) .

Lalor, E., "Conditions for the Validity of the Angular Spectrum of Plane Waves," J. Opt. Soc. Am. 58:1235 (1968).

Leinert, C., and D. Klüpelberg, "Stray Light Suppression in Optica1 Space Experiments," App1. Opt. 13:556 (1974). 
McKenney, D. B., G. R. Orme, and L. P. Mott, "Light Scattering by Thin Film Coatings," Final Report, Optical Sciences Center, University of Arizona, Tucson (1972).

Moffitt, F. H., Photogrammetry, International Textbook Co. (1959).

Mott, L. P., The Effect of Surface Roughness on the Optical Properties of All-dielectric Interference Filters, Master's thesis, University of Arizona, Tucson (1971).

Nankive11, J. F., "The Theory of Electron Stereo Microscopy," Optic 20: 171 (1963).

Nicodemus, F. E., "Reflectance Nomenclature and Directional Reflectance and Emissivity," App1. Opt. 9:1474-5 (1970).

Orme, G. R., "Measurement of Small Angle Scatter from Smooth Surfaces," Optical Sciences Center Tech. Rpt. 74, University of Arizona, Tucson (1972).

Papoulis, A., Probability, Random Variables, and Stochastic Processes, McGraw-Hil1, N. Y. (1965).

Peake, W. H., "Interaction of Electromagnetic Waves with Some Natural Surfaces," IRE Trans. AP-7 (1959).

Perkin-Elmer Final Report prepared for George C. Marshall Space Flight Center under Contract NAS8-30638, "Study of U1traviolet and Visible Scattered Light Effects on the Optical Performance of the Large Space Telescope," (1975).

Porteus, J. 0., "Relation between the Height Distribution of a Rough Surface and the Reflectance at Normal Incidence," J. Opt. Soc. Am. 53:1394-1402 (1963).

Ratcliffe, J. A., "Some Aspects of Diffraction Theory and Their Application to the Ionosphere," in A. C. Strickland, ed., Reports on Progress in Physics, Vol. XIX, The Physical Society, London (1956).

Rayleigh, Lord, "Polish," Nature 64:385-388 (1901).

Rayleigh, Lord, The Theory of Sound, Vol. II, Dover, N. Y. (1945).

Scheele, S. R., Scattering Characteristics of Mirrors and the Associated Inverse Scattering Problem, Ph. D. dissertation, University of California at Los Angeles (1973). 
Shack, R. V., "Interaction of an Optical System with the Incoming Wavefront in the Presence of Atmospheric Turbulence," Optical Sciences Center Tech. Rpt. 19, University of Arizona, Tucson (1967).

Shack, R. V., and M. A. DeBe11, "Surface Scatter Study," Optical Sciences Center final report prepared for the Space and Missile Systems Organization under Contract F04701-72-C-0181, University of Arizona, Tucson (1974).

Shack, R. V., and J. E. Harvey, "An Investigation of the Distribution of Radiation Scattered by Optical Surfaces," Optical Sciences Center final report prepared for the Space and Missile Systems Organization under Contract F04701-74-C-0083, University of Arizona, Tucson (1975).

Shevlin, C. M., Department of Metallurgy, University of Arizona, Tucson, Private Communication (1974).

Spetner, L. M., "A Statistical Model for Forward Scattering of Waves of a Rough Surface," Trans. IRE AP $\underline{6}: 88-94$ (1958).

Stover, J. C., "Roughness Characterization of Smooth Machined Surfaces by Light Scattering," App1. Opt. 14:1796 (1975).

Thompson, S. P., Treatise on Light, Macmillan and Co., London (1912).

Twersky, V., "On Scattering and Reflection of Electromagnetic Waves by Rough Surfaces," IRE Trans., PP. 81-90 (1957).

Vachaspati, "Scattering of Light by Free Electrons, Intensity Dependent Phase Shift and Frequency Change," Indian J. Pure and App1. Phys. 2:373-376 (1964).

Wey1, H., "Ausbreitung elektromagnetischer wellen uber einen ebenen leiter," Ann. Phys. 60:481-500 (1919).

Young, R. P., "Metal Optics Scatter Measurements," Oral Presentation at SPIE's 19th Annual International Technical Symposium, San Diego, CA., August (1975). 